\title{
Dynamic Temperature Mapping - Real-time Strategies and Model-based Reconstructions
}

\author{
Dissertation \\ for the award of the degree \\ "Doctor rerum naturalium" \\ of the Georg-August-Universität Göttingen
}

within the doctoral program Physics of Biological and Complex Systems of the Georg-August University School of Science (GAUSS)

submitted by

Zhongshuai Zhang

from Changchun, China

Göttingen, 2016 


\section{Thesis Committee}

Prof. Dr. Jens Frahm

Biomedizinische NMR Forschungs GmbH, Max-Planck-Institut für biophysikalische Chemie

Prof. Dr. Jörg Enderlein Drittes Physikalisches Institut, Georg-August-Universität Göttingen

Prof. Dr. Stefan Luther

Biomedizinische Physik,

Max-Planck-Institut für Dynamik und Selbstorganisation

\section{Members of the Examination Board}

Prof. Dr. Jens Frahm

(Referee)

Prof. Dr. Jörg Enderlein

( $2^{\text {nd }}$ Referee)
Biomedizinische NMR Forschungs GmbH, Max-Planck-Institut für biophysikalische Chemie

Drittes Physikalisches Institut, Georg-August-Universität Göttingen

\section{Further members of the Examination board}

Prof. Dr. Stefan Luther

Dr. Peter Dechent

Prof. Dr. Marina Bennati

Prof. Dr. Helmut Grubmüller
Biomedizinische Physik, Max-Planck-Institut für Dynamik und Selbstorganisation

Abtl. Kognitive Neurologie, Universitätsmedizin Göttingen

AG Elektronspinresonanz-Spektroskopie, Max-Planck-Institut für biophysikalische Chemie

Abtl. Theoretische und computergestützte Biophysik, Max-Planck-Institut für biophysikalische Chemie

Date of oral examination: December 14, 2016 


\section{Acknowledgements}

First of all, I am very grateful to Prof. Dr. Jens Frahm, head of the Biomedizinische NMR Forschungs GmbH am Max-Planck-Insitut für biophysikalische Chemie. It is a great honor and pleasure to study under his supervision. I would not achieve my thesis without his continuous support. His great passion for science always inspires me during my whole PhD study.

I would like to thank Prof. Dr. Jörg Enderlein and Prof. Dr. Stefan Luther for their supervision and interest in my thesis. I also would like to thank Dr. Klaus-Dietmar Merboldt and Dr. Thomas Michaelis for the invaluable scientific suggestions and discussions.

I would like to thank Dr. Roland Tammer for the contribution to the temperature phantom.

I thank all the members of the Biomedizinische NMR Forschungs GmbH for creating the excellent working atmosphere. I would alway remember the parties and beers we had together, especially the kicker games we had everyday.

Further, I would like to thank all my friends in Germany and China for their encouragement and support during my four years of study.

I thank Dr. Thomas Michaelis for offering me the great help with improving the quality of my thesis. I also thank Dr. Dirk Voit, Dr. Zhengguo Tan and Dana Dedeck for improving my writing skill.

Finally, I am indebted to my wife and my parents. I thank for your support, encouragement, company, and love. 



\section{Contents}

1. Introduction 1

2. Magnetic Resonance Imaging 3

2.1. NMR Phenomenon . . . . . . . . . . . . . . . . . . 3

2.1.1. Excitation . . . . . . . . . . . . . . . 4

2.1.2. Relaxation Effects. . . . . . . . . . . . . . . 5

2.2. Free Induction Decay and Gradient Echo . . . . . . . . . . . . . . . . 7

2.2.1. Free Induction Decay . . . . . . . . . . . . . . . . . 7

2.2.2. Gradient Echo . . . . . . . . . . . . . . . . . . . . 7

2.3. Spatial Encoding . . . . . . . . . . . . . . . . . 8

2.3.1. Slice Selection . . . . . . . . . . . . . . . . . . . . 8

2.3.2. k-Space Sampling . . . . . . . . . . . . . . . . . . . . . . . 9 9

2.4. Sampling Requirements . . . . . . . . . . . . . . . . . . . . . . 12

2.5. Real-time MRI . . . . . . . . . . . . . . . . . . . 12

2.5.1. Radial FLASH Sequence . . . . . . . . . . . . . . . . . . . 13

2.5.2. Data Undersampling \& Nonlinear Inverse Reconstruction . . . . . 14

3. Laser Induced Thermal Therapy and MR Thermometry Methods 19

3.1. Laser Induced Thermal Therapy . . . . . . . . . . . . . . . . . . . . . . . 19

3.1.1. Laser . . . . . . . . . . . . . . . . . . . . . . . . . . . 19

3.1.2. Heat Transfer in Tissue . . . . . . . . . . . . . . . . . . . . 21

3.2. MR Thermometry Methods . . . . . . . . . . . . . . . . . 23

3.2.1. Proton Resonance Frequency . . . . . . . . . . . . . . . . . 23

3.2.2. $\mathrm{T}_{1}$ Relaxation Time . . . . . . . . . . . . . . . 24

3.2.3. Alternative Temperature-sensitive MR Parameters . . . . . . . . . 25

4. Real-time MR Thermometry - Phantom Studies 27

4.1. Phantom Development . . . . . . . . . . . . . . . . 27

4.2. PRF Thermometry . . . . . . . . . . . . . . . . . . 28

4.2.1. Methods and Materials . . . . . . . . . . . . . . . 28

4.2.2. Results . . . . . . . . . . . . . . . . . . 34

4.3. $\mathrm{T}_{1}$ Thermometry . . . . . . . . . . . . . . . . . . 38

4.3.1. Methods and Materials . . . . . . . . . . . . . . . 38

4.3.2. Results . . . . . . . . . . . . . . . . . . . . 41 


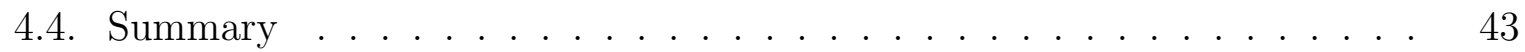

5. Real-time MR Thermometry - Ex Vivo Tissue Studies with Global Heating 47

5.1. Comparison between PRF Shift and $T_{1}$ Methods . . . . . . . . . . . . . 48

5.1.1. Experimental Setup . . . . . . . . . . . . . . . . . . . . 48

5.1.2. Results . . . . . . . . . . . . . . . . . . 48

5.1.3. Summary ........................ 50

5.2. Evaluation of PRF Method in Ex Vivo Tissue . . . . . . . . . . . . 54

5.2.1. Experimental Setup . . . . . . . . . . . . . . 58

5.2.2. Results . . . . . . . . . . . . . . . . 60

5.2.3. Discussion . . . . . . . . . . . . . . 60

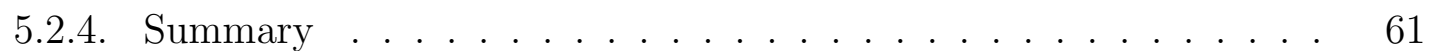

6. Real-time MR Thermometry - Ex Vivo Tissue Studies with Focal Heating $\mathbf{6 5}$

6.1. MRI Sequence Optimization . . . . . . . . . . . . . . . . 65

6.2. Dynamic Temperature Monitoring during Laser Heating . . . . . . . . 67

6.2.1. Laser Power \& Temperature . . . . . . . . . . . . . . . . . . . 67

6.2.2. Determination of 'Black Hole' . . . . . . . . . . . . . . . . . 68

6.2.3. Single Laser Pulse \& Temperature . . . . . . . . . . . . . . . . . . 73

6.2.4. Laser Experiments in Kidney Medulla . . . . . . . . . . . . . 75

6.2.5. Laser Protocols under Minimally Invasive Surgery Conditions . . 78

6.3. Summary . . . . . . . . . . . . . . . . . . . 80

7. Reference Choice under In Vivo Conditions 83

7.1. Introduction . . . . . . . . . . . . . . . . . . . 83

7.2. Methods and Materials . . . . . . . . . . . . . . . . . 84

7.3. Results . . . . . . . . . . . . . . . . . . 85

7.4. Summary . . . . . . . . . . . . . . . . 86

8. Model-based Reconstruction for MR Thermometry 91

8.1. Introduction . . . . . . . . . . . . . . . . . . . 91

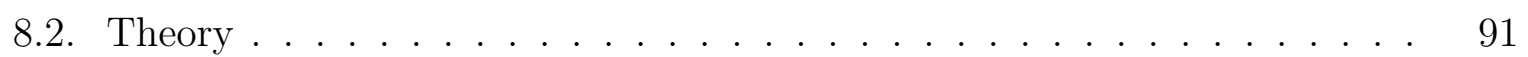

8.2.1. PRF MR Thermometry as Nonlinear Inverse Problem . . . . . . . 91

8.2.2. Regularization . . . . . . . . . . . . . . . . 93

8.3. Methods and Materials . . . . . . . . . . . . . . . . . . . . 94

8.3.1. Numerical Simulation . . . . . . . . . . . . . . . . . . . . 94

8.3.2. Laser Heating in Ex vivo Kidney . . . . . . . . . . . . . . . 94

8.4. Results . . . . . . . . . . . . . . . . . . . . . 95

8.4.1. Numerical Simulation . . . . . . . . . . . . . . . . . . . . 95

8.4.2. Laser Heating Experiments . . . . . . . . . . . . . . . . . . . . 95

8.5. Summary . . . . . . . . . . . . . . . . . . . . . 97 
$\begin{array}{ll}\text { 9. Summary and Outlook } & 101\end{array}$

9.1. Summary . . . . . . . . . . . . . . . . . . . . . . . . 101

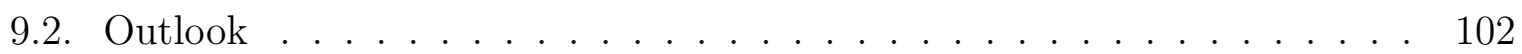

Appendix A. Calculation of Bio-heat Transfer Model 105

$\begin{array}{ll}\text { Bibliography } & 107\end{array}$

$\begin{array}{ll}\text { Curriculum Vitae } & 117\end{array}$ 



\section{Introduction}

Magnetic resonance imaging (MRI) is a medical imaging, modality which is widely used for research as well as diagnostic purposes. The technique relies on the radiofrequency (RF) excitation of proton spins and RF signal detection during relaxation to equilibrium. MRI benefits from the presence of abundant hydrogen atoms in human body and offers soft-tissue contrast with astonishing quality. Compared with other diagnostic modalities such as X-ray computed tomography (CT) or positron emission tomography (PET), MRI may be performed without exposing patients to ionizing radiation. Apart from providing anatomic views in arbitrary orientations, MRI offers a wide range of different sequences which allow for tissue characterizations by diverse parameters including proton density, $T_{1}, T_{2}$, flow velocity, diffusion coefficient and temperature.

On the other hand, the traditional MRI scan is time-consuming when compared to X-ray CT. Conventional acquisitions with long measuring times therefore encounter problems (e.g., image artifacts) when monitoring moving organs such as the beating heart or the liver during breathing. Moreover, rapid non-periodic physiologic processes ranging from speaking to swallowing are not accessible at all. Consequently, tremendous efforts have been dedicated to improve the temporal resolution. While early trials refer to data acquisitions by echo-planar imaging, more recent attempts involve non-Cartesian encoding techniques and the use of multiple $\mathrm{RF}$ receive coils for parallel imaging. This latter approach exploits the complementary spatial information from multi-channel receive coils that are distributed around the object or body part of interest.

Meanwhile, minimally invasive thermal therapies for hyperthermia or coagulation of tissue have received clinical interest during the last decades. Such thermal treatments represent alternatives to conventional surgeries and typically aim to coagulate tumor tissue by increasing the local temperature beyond a certain threshold. However, in order to ensure both safety and efficacy of thermal therapies, it is mandatory to monitor the local temperature during the entire heating process. MRI is particularly suitable for meeting this challenge, as it not only provides a variety of temperature-sensitive parameters, but also offers spatial mapping of the temperature distribution. The key challenge of dynamic temperature mapping is to achieve high spatiotemporal resolution. Parallel imaging such as generalized autocalibrating partially parallel acquisitions (GRAPPA) and autocalibrating variants of sensitivity encoding (SENSE) only moderately improved the imaging speed 


\section{Introduction}

by a factor of $2-3$, which in many thermometry studies will not be sufficient or lead to lowered spatial resolution. Recently, Uecker et al. [1] reported significant advances in the development of real-time MRI. The technical progress is based on highly undersampled radial gradient-echo sequences in conjunction with a novel reconstruction technique that defines the image as the solution of a nonlinear inverse (NLINV) problem. Because dynamic imaging, i.e. the successive acquisition and reconstruction of serial images at high temporal resolution, is characterized by a certain degree of temporal continuity, the mathematical algorithm was complemented by temporal regularization to the preceding frame.

The purpose of this thesis was to explore the potential of real-time MRI methods for temperature mapping during thermal procedures. This mainly included a comprehensive analysis of two different methods, i.e. proton resonance frequency (PRF) and $T_{1}$ thermometry, with respect to speed, robustness, and accuracy in different experimental settings. These methods were chosen because of their pronounced temperature sensitivities and relatively rapid data sampling schemes. Basic implementations and comparisons of suitable data acquisition and image reconstruction strategies were accomplished with use of a home-made temperature phantom. Secondly, PRF thermometry as the resulting preferred method was further analyzed using heating studies of ex vivo tissues. And thirdly, in order to even better mimic clinical thermal therapies, focal heating with use of a laser system was studied on swine kidneys. In both kidney cortex and medulla, the temperature changes for different output powers could be monitored by PRF thermometry in real time. A further development in preparation of future in vivo studies referred to an optimized reference selection procedure in order to minimize temperature errors in the PFR method due to inter-scan motion between the reference and actual scan. Lastly, a model-based reconstruction approach for phase-contrast MRI, which yields temperature maps and anatomic images directly from undersampled raw data sets, has been adopted to the present conditions. Efforts were made especially with respect to temporal regularization to obtain temperature maps with considerably increased quality. 


\section{Magnetic Resonance Imaging}

In the first part of this chapter, basic principles of MRI are introduced concerning the aspects of nuclear physics, signal generation, and traditional image reconstruction. More comprehensive knowledge is referred to $[2,3]$. The second part gives a brief overview of real-time MRI technique, which is considered as a prerequisite for the dynamic MR thermometry.

\subsection{NMR Phenomenon}

Nuclear magnetic resonance (NMR) phenomenon was first discovered by Purcell and Bloch in 1946 [4, 5], which can be observed in the nuclei with non-zero spin quantum number, e.g. ${ }^{1} \mathrm{H},{ }^{13} \mathrm{C},{ }^{19} \mathrm{~F}$, and ${ }^{23} \mathrm{Na}$. MRI often uses the signal generated from ${ }^{1} \mathrm{H}$, due to its abundant presence in water, fat and other molecules in the human body. The relationship between the magnetic moment $\mu$ and the proton spin angular momentum $(I)$ is given by

$$
\mu=\gamma I
$$

where $\gamma$ is the so called gyromagnetic ratio, a proportionality constant. Due to the fact that the quantum number of hydrogen protons is $1 / 2$, the hydrogen protons positioned in an external magnetic field are splitted into two possible quantum spin states, which are $E_{\uparrow}$ (spins align parallel to the external magnetic field) and $E_{\downarrow}$ (spins align anti-parallel to the external magnetic field) and can be expressed as:

$$
\begin{aligned}
E_{\uparrow} & =-\gamma \frac{\hbar}{2} B_{0} \\
E_{\downarrow} & =\gamma \frac{\hbar}{2} B_{0}
\end{aligned}
$$

where $\hbar$ is the Planck's constant divided by $2 \pi, B_{0}$ represents the external magnetic field. The energy difference between the states above is then:

$$
\Delta E=\gamma \hbar B_{0}
$$


Additionally, according to the Boltzmann distribution, placing nuclear spins in a thermal equilibrium system, the population density $P(E)$ of each spin energy level is described as:

$$
P(E) \propto e^{-E / k T}
$$

where $k$ is the Boltzmann constant and $T$ is the absolute temperature. Substituting Equation (2.2), (2.3) into Equation (2.5), the population ratio between the two energy states can be expressed as:

$$
\frac{N_{\uparrow}}{N_{\downarrow}}=e^{\Delta E / k T}
$$

In order to calculate the bulk magnetization $\left(M_{0}\right)$, one could take thermal interaction and the quantum basis of the magnetization into account, the corresponding expression is

$$
M_{0}=\rho \frac{\gamma^{2} \hbar^{2}}{4 k T} B_{0}
$$

where $\rho$ is the proton density. The formula above is derived under the assumption that the nuclear magnetic energy is much smaller than the thermal energy (at human body temperature). Curie's law also experimentally proves that magnetization is inverse proportional to the absolute temperature.

\subsubsection{Excitation}

In the present work, the direction of the external magnetic field $\left(\overrightarrow{B_{0}}\right)$ is defined as the longitudinal $(z)$ direction and its perpendicular plane is defined as the transversal $(x-y)$ plane, thus the magnetic moment vector precesses around the $z$ axis with a fixed angle and the procession frequency is expressed as:

$$
\omega=\gamma B_{0}
$$

where $\omega$ is the Larmor frequency. For hydrogen protons, the gyromagnetic ratio is $2.68 \times$ $10^{8} \mathrm{rad} /(\mathrm{s} \cdot$ Tesla). Therefore, take the MRI system employed in this thesis as an example (Magnetom Prisma, Siemens Healthcare, Erlangen, Germany), the static magnetic field $\left(\mathrm{B}_{0}\right)$ is $2.89 \mathrm{~T}$ and the corresponding Larmor frequency $(\omega)$ is $7.74 \times 10^{8} \mathrm{rad} / \mathrm{s}$ or $f_{0}$ is $123.27 \mathrm{MHz}$. 
In a spin 'isochromat' volume, a large number of protons are considered to be placed in a static homogeneous magnetic field. Neglecting the proton interactions, the total motion of the individual spin is:

$$
\frac{d \vec{M}}{d t}=\gamma \vec{M} \times \vec{B}_{0}
$$

By applying a radio frequency $(\mathrm{RF})$ field $\left(\vec{B}_{1}\right)$ in $x$-y plane with Larmor frequency $(\omega)$, spins are excited. The magnetization is flipped from the longitudinal axis towards the transverse plane with a flip angle $\alpha$ which is proportional to the integral over the applied RF field $\vec{B}_{1}[6]$ :

$$
\alpha=\gamma \int_{0}^{T_{R F}} B_{1}(t) d t
$$

where $T_{R F}$ is the duration of the RF pulse. As a result, the superimposed magnetic field $\vec{B}(t)$ can be described as:

$$
\vec{B}(t)=\vec{B}_{0}+\vec{B}_{1}(t)=\left(\begin{array}{c}
0 \\
0 \\
\vec{B}_{0}
\end{array}\right)+\left(\begin{array}{c}
\vec{B}_{1}(t) \sin (\omega t) \\
\vec{B}_{1}(t) \cos (\omega t) \\
0
\end{array}\right)
$$

\subsubsection{Relaxation Effects}

After the RF excitation, the moment of spins, which aligns with the superimposed field $\vec{B}(t)$, will precess towards the thermal equilibrium state through two relaxation mechanisms, longitudinal relaxation and transversal relaxation.

The longitudinal relaxation (along $z$ axis) is also known as the spin-lattice relaxation. The regrowth rate of the longitudinal magnetization $\left(d M_{z} / d t\right)$ is proportional to the magnetization difference during the recovery time and this relationship can be described as:

$$
\frac{d M_{z}}{d t}=\frac{1}{T_{1}}\left(M_{0}-M_{z}\right)
$$

where $T_{1}$ is the spin-lattice or the longitudinal relaxation time and $M_{0}$ is the magnetization in the static magnetic field $\left(\vec{B}_{0}\right)$. The numerical solution of Equation $(2.12)$ is given by:

$$
M_{z}(t)=M_{z}(0)\left(1-e^{-\frac{t}{T_{1}}}\right)
$$


The transversal relaxation (in $x$-y plane) is also called the spin-spin relaxation. The magnetization decay rate in $x-y$ plane $\left(d M_{x y} / d t\right)$ is given by:

$$
\frac{d M_{x y}}{d t}=-\frac{M_{x y}}{T_{2}}
$$

where $T_{2}$ is the spin-spin or the transverse relaxation time, $M_{x y}$ is the magnetization residue in $x-y$ plane. The numerical solution of Equation (2.14) is:

$$
M_{x y}=M_{0} e^{-\frac{t}{T_{2}}}
$$

In 1946, Bloch extended $T_{1}$ and $T_{2}$ relaxations into a one vector equation [4] which is written as:

$$
\frac{d \vec{M}}{d t}=\vec{M} \times \gamma \vec{B}+\left(\begin{array}{c}
-M_{x} / T_{2} \\
-M_{y} / T_{2} \\
\left(M_{0}-M_{z}\right) / T_{1}
\end{array}\right)
$$

Both $T_{1}$ and $T_{2}$ are tissue specific constant parameters with a variation of $400-1500 \mathrm{~ms}$ and 50-150 ms, respectively and thus these two parameters offer a great opportunity to distinguish different tissue types in the human body. Figure 2.1 shows the precession of the magnetization after the RF excitation in a rotating frame.

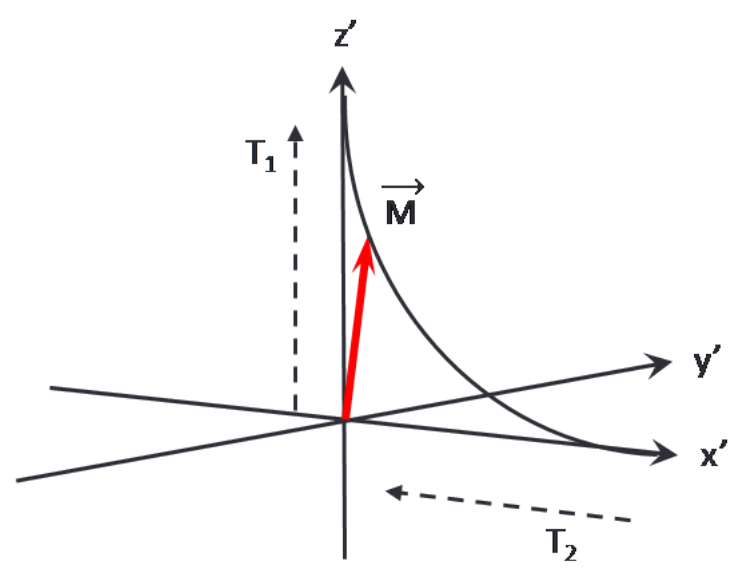

Figure 2.1.: Relaxation mechanisms of the magnetization in a rotating frame. The red arrow represents the current magnetization which relaxes from two aspects: $T_{2}$ decay along x' axis and $T_{1}$ recovery along z' axis. 


\subsection{Free Induction Decay and Gradient Echo}

\subsubsection{Free Induction Decay}

Practically, protons are located in an inhomogeneous magnetic field, which results in a increased spin dephasing in the transverse plane [7]. Thus, effective spin-spin relaxation time $\left(T_{2}^{*}\right)$ is shorter than the ideal $T_{2}$. Such a experimental phenomenon is called free induction decay (FID) and the relationship between $T_{2}^{*}$ and $T_{2}$ is given by:

$$
\frac{1}{T_{2}^{*}}=\frac{1}{T_{2}}+\gamma \Delta B_{0}
$$

with $\Delta B_{0}$ representing the local magnetic field inhomogeneity.

\subsubsection{Gradient Echo}

After the RF excitation, spins are dephasing under the influence of FID. By introducing a gradient magnetic field along $z$ axis, one can achieve a more rapid dephasing of spins. Another gradient with opposite polarity is followed with the previous one for the purpose of generating an echo (seen in Figure 2.2). Due to the combined influence of magnetic field inhomogeneities and $T_{2}$ relaxation, detected signal intensity decays with echo time (TE) by a factor of $e^{\left(-\mathrm{TE} / T_{2}^{*}\right)}$. Gradient echo is often used in fast imaging technique, and also the base of fast low angle shot (FLASH) sequence $[8,9]$.

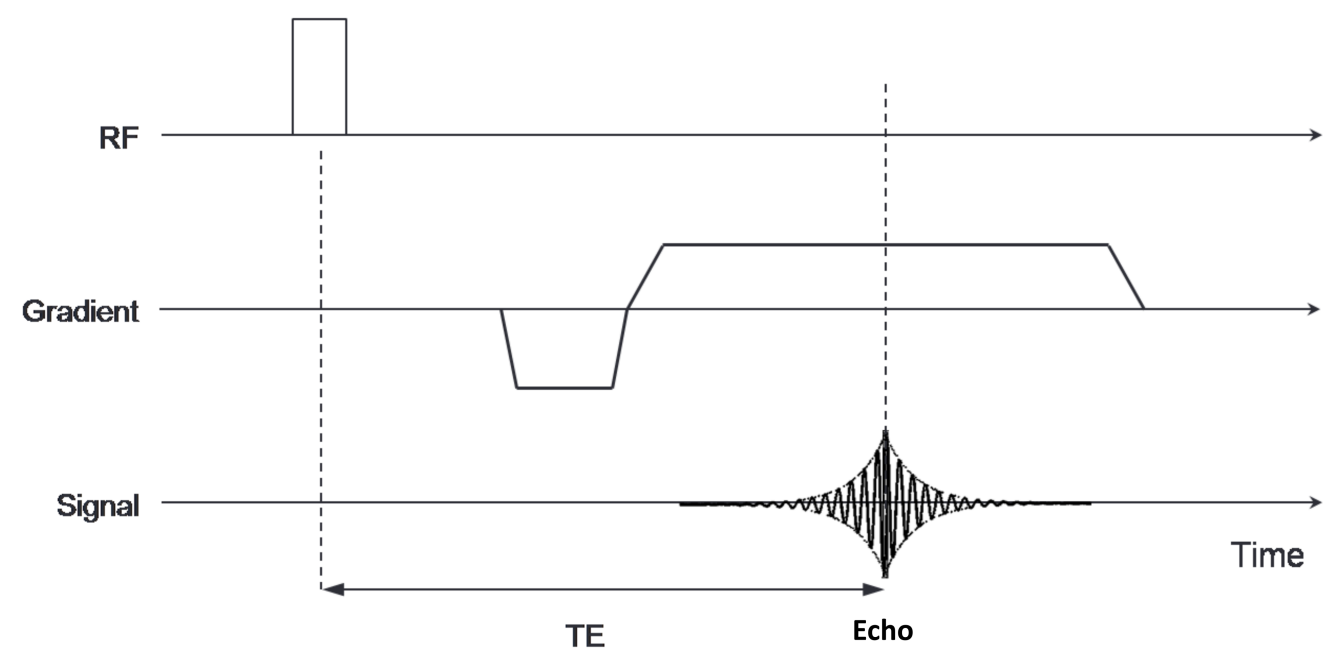

Figure 2.2.: Gradient echo: A dephasing gradient followed by an echo-recall gradient for signal generation. 


\subsection{Spatial Encoding}

If one only applies an uniformed RF pulse in the target volume, all the spins would process in the same resonance frequency (assuming in a homogeneous magnetic field). In order to distinguish spins from different spatial locations for two dimensional (2D) or three dimensional (3D) imaging, P. Lauterbur, one of the Nobel Prize winners in 2003, introduced an idea that acquires signal by applying additional magnetic gradient fields along different directions [10]. Therefore, the superimposed spatial magnetic field of a desired location can be written as:

$$
\vec{B}(x, y, z)=\vec{B}_{0}+\vec{G}_{x} \cdot x+\vec{G}_{y} \cdot y+\vec{G}_{z} \cdot z
$$

where $\vec{G}_{x}, \vec{G}_{y}, \vec{G}_{z}$ represent different gradient directions (along $x, y$ and $z$ axis, respectively), the units of the gradient field is usually measured in $\mathrm{mT} / \mathrm{m}$.

In the present work, attentions are focused on 2D MR images, therefore two necessary gradients are required: the slice selection gradient and the Fourier encoding gradient.

\subsubsection{Slice Selection}

For convenience, it is assumed that the 2D image is located in the $x-y$ plane and the thickness of the chosen slice is along the $z$ axis. In order to only excite spins in desired slice, a linear gradient in $z$ direction and a RF pulse with a certain bandwidth are combined.

According to Equation (2.18), under a gradient magnetic field, the spin resonance frequency becomes spatial dependent:

$$
\omega(z)=\omega_{0}+\gamma G_{z}(z)
$$

Meanwhile, assume the desired slice thickness is $\Delta z$, the frequency range of the RF pulse should be from $\gamma G_{z} z_{0}-\gamma G_{z} \Delta z / 2$ to $\gamma G_{z} z_{0}+\gamma G_{z} \Delta z / 2$ and the relationship between bandwidth $(\mathrm{BW})$ and $\Delta z$ is expressed as:

$$
\begin{aligned}
B W & =\left(\gamma G_{z} z_{0}-\gamma G_{z} \Delta z / 2\right)-\left(\gamma G_{z} z_{0}+\gamma G_{z} \Delta z / 2\right) \\
& =\gamma G_{z} \Delta z
\end{aligned}
$$

As demonstrated in Equation (2.20), it is clear that both BW and the gradient field can influence the slice thickness (also seen in Figure 2.3). Ideally, the hard RF pulse would 
be suitable for a perfect slice excitation, the inverse Fourier transform of which is an infinite sinc function in the spatial domain. However, not all the side lobes of the sinc function can be included when applying the RF pulse, leading to an imperfect slice profile in practice.

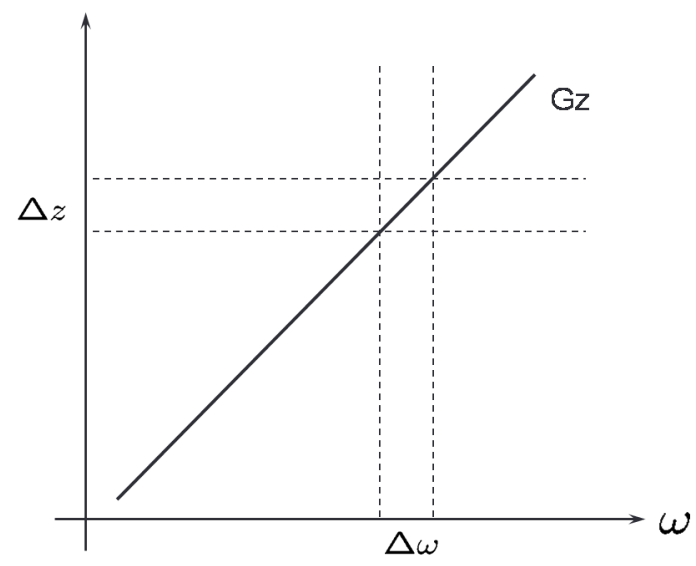

Figure 2.3.: The relationship between the bandwidth of the RF pulse and the slice thickness. By switching the slope of the gradient or the RF bandwidth can manipulate the slice thickness.

\subsection{2. k-Space Sampling}

After the slice selective excitation, the desired spins are flipped by a certain angle (e.g. $90^{\circ}$ ) onto the $x-y$ plane. The remaining issue is to distinguish the signal from targeted spins within the two dimensional region. Here, Cartesian trajectory is used as an example to introduce data sampling and the related technique to acquire spatial information is the so called Fourier encoding that consists of the frequency and the phase encoding.

\section{Frequency Encoding}

Focusing on one line along the $x$ axis of the measured 2D sample and without considering the proton relaxation process for simplification reasons, under the static magnetic field, the Larmor frequency of the rotating spins is $\omega_{0}$. Similar to Equation (2.19), if a gradient along the $x$ axis $\left(G_{x}\right)$ is applied during signal acquisition interval, the corresponding Larmor frequency can be written as:

$$
\omega(x)=\gamma\left(B_{0}+G_{x}(x)\right)
$$

Therefore, the detected complex signal is proportional to the magnetization in the transverse plane and can be expressed by a complex model: 


$$
s(t) \propto M(x, t)=M_{0}(x) \cdot e^{-i \omega(x) t}
$$

where $M_{0}(x)$ is the magnitude of the magnetization at the position $x$ and $t$ represents the total time interval of $G_{x}$. Substituting Equation (2.21) into (2.22), the signal of the sampled one dimensional line can be described as:

$$
s(t) \propto \int M_{0}(x) \cdot e^{-i\left(\omega_{0}+\gamma G_{x}(x)\right) t}
$$

which indicates that both static field $B_{0}$ and applied gradient $G_{x}$ contribute to the phase accumulation.

\section{Phase Encoding}

By employing frequency encoding gradient of the desired slice, only one dimensional signal can be acquired. In order to cover the whole 2D slice, another gradient (along $y$ axis) must be applied to switch the acquisition from one 'line' to another. Such a gradient is called phase encoding gradient.

Phase encoding gradient $\left(G_{y}\right)$ is activated between the slice excitation and the frequency encoding gradient with a short duration to generate a unique precession frequency of a specific 'line'. After turning off the gradient, the initial phase before the frequency encoding is given by:

$$
\phi(y)=\gamma \int_{0}^{T} y G_{y}(t) d t
$$

where $T$ is the total duration of $G_{y}$. The phase encoding gradient is introduced repetitively with different strength to achieve encoding different one dimension 'lines' in the target slice.

\section{k-Space Sampling}

As for two dimensional encoding, one can combine Equations (2.23) and (2.24) to derive the relationship between the obtained signal $S$ and the sampled location $p$ :

$$
S(p)=\iint M(p) \cdot e^{-i 2 \pi(k(t) \cdot p)} d p
$$

where $k(t)$ is defined as k-space position, with:

$$
k(t)=\frac{\gamma}{2 \pi} \int_{0}^{t} G(t) d t
$$


To be more specific, by varying and combining different gradient fields, the sampled object in spatial domain is encoded in the frequency domain via Fourier transformation. Equation (2.25) indicates that MRI signal is equivalent to the Fourier transform of the magnetization. According to Equation (2.26), the k-space position, $k(t)$, represents the sampled frequency at time $t$ during data acquisition.

\section{Cartesian Trajectory}

The conventional scheme of sampling the k-space data is a line-by-line pattern, which is referred as the Cartesian trajectory. As discussed in the previous part of this section, frequency encoding is applied to acquire one single 'line' in k-space and phase encoding is applied to shift one 'line' to another in order to cover the whole k-space. Figure 2.4 illustrates the Cartesian sampled sequence and the corresponding k-space trajectory. The time period between two consecutive RF excitations is defined as repetition time (TR), and the duration from RF pulse center to the echo is defined as echo time (TE).

Nowadays, due to the improvement of MRI hardware, more alternative k-space trajectories are also developed for clinical practice, such as echo-planar imaging [11, 12], spiral $[13,14]$ and radial [10] schemes. More details about the radial sampling pattern, which is adopted in real-time MRI, will be introduced later in this chapter.

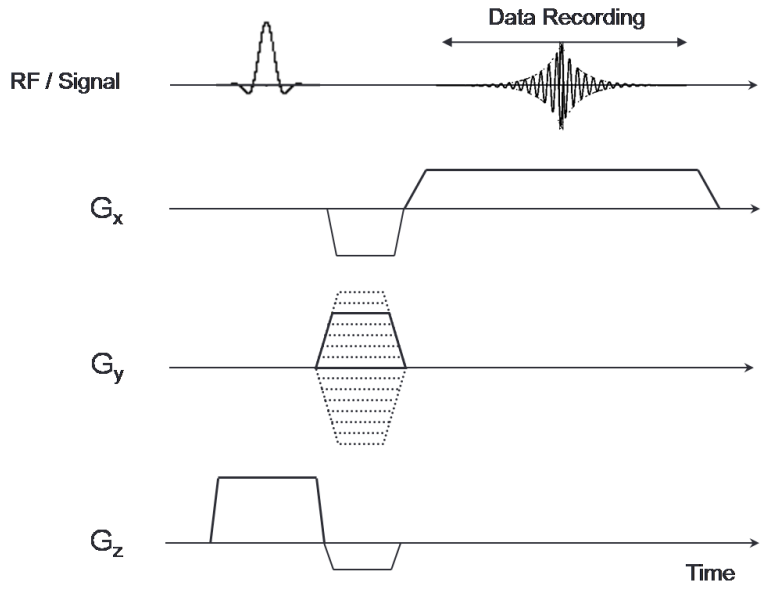

(a)

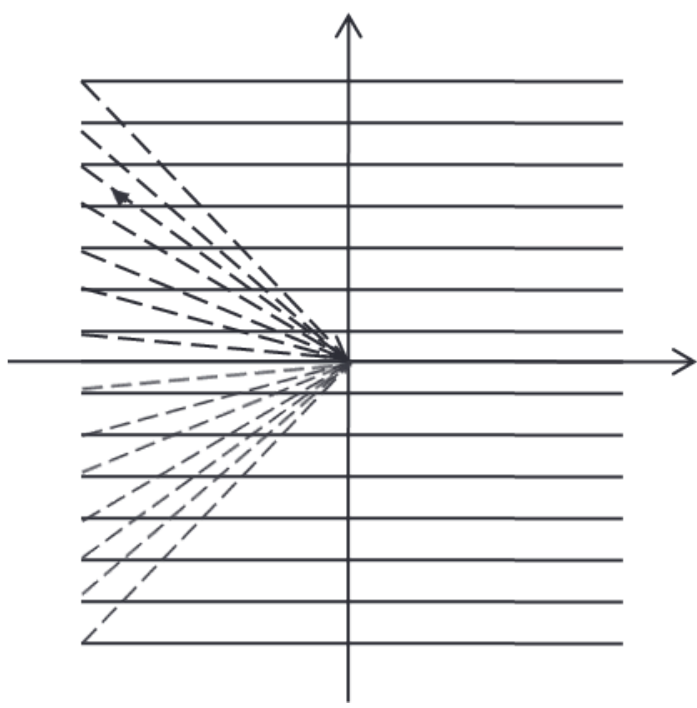

(b)

Figure 2.4.: (a) Sequence timing diagram of a 2D Cartesian acquisition. (b) The corresponding sampling trajectory. 


\subsection{Sampling Requirements}

Practically, the MR signal is sampled in a discretized way with a certain sampling rate and thus the target $2 \mathrm{D} \mathrm{k}$-space is formulated as gridded points. The distance between the two neighboring discrete points, $\Delta k$, in a given field of view (FOV) should fulfill the following relationship:

$$
\Delta k=\frac{1}{F O V}
$$

In order to increase the signal to noise ratio (SNR), a smaller $\Delta k$ is preferred for data acquisition. Meanwhile, the sampling interval or the dwell-time of the signal receivers $(\Delta t)$ is inversely proportional to the readout bandwidth $(\mathrm{BW})$, shown as:

$$
\Delta t=\frac{1}{B W}
$$

Equation (2.28) shows that a higher BW corresponds to a shorter sampling interval which leads to a low image SNR. Conversely, a lower BW corresponds to a longer sampling interval which leads to a high image SNR. With a given FOV, the spatial resolution $(\Delta x)$ is determined by the total sampled points or the base resolution (n) and the corresponding relationship is expressed as:

$$
\Delta x=\frac{\mathrm{FOV}}{n}
$$

In order to prevent aliasing artifacts due to insufficient sampling distance, Nyquist criterion has to be satisfied:

$$
\Delta k \leq \frac{1}{\mathrm{FOV}}
$$

Generally, based on the requirements mentioned above, one can reconstruct images directly from the Cartesian sampled k-space data by applying inverse Fast Fourier Transformation (FFT) [15].

\subsection{Real-time MRI}

Nowadays, more attention is paid on developing rapid imaging techniques to reduce motion artifacts for monitoring fast physiological processes without breath holding and/or cardiac gating. In this section, real-time MRI is introduced, which is a combination of the 
radial FLASH data acquisition and the nonlinear inverse image reconstruction algorithm.

\subsubsection{Radial FLASH Sequence}

Fast low angle shot (FLASH) technique, which is a gradient echo sequence, was first proposed in 1985 [8, 9]. Instead of using intense RF excitation pulses, FLASH provides $T_{1}$ weighted images [16] by tilting the rotating spins with a low angle to reduce the scanning time without sacrificing the image SNR and the spatial resolution. Generally, FLASH sequence is used to obtain images in steady state [17]. RF spoiling [18] and/or gradient spoiling [19] are also commonly applied to overcome the problem caused by the incomplete magnetization dephasing in the transverse plane. Moreover, to improve the temporal resolution, T. Block [20] and S. Zhang [21] extended FLASH into radial FLASH from the perspective of data sampling trajectory.

Figure 2.5 shows the radial sampling trajectory in k-space. Each trajectory line (spoke) crosses the $\mathrm{k}$-space center. This trajectory is achieved by varying the gradient amplitudes in both $x$ and $y$ axises:

$$
\begin{aligned}
G_{x} & =G_{\text {max }} \cdot \cos \theta \\
G_{y} & =G_{\text {max }} \cdot \sin \theta
\end{aligned}
$$

where $G_{\max }$ represents the maximum gradient amplitude, and $\theta$ denotes the angle of the corresponding spoke. Even though radial trajectory is proposed by P. Lauterbur [10] as early in 1973, it has only regained major interests since the last two decades as radial sampling has higher technical requirements. On one hand, higher $B_{0}$ field homogeneity and precise timing of the gradient fields are demanded. On the other hand, instead of applying FFT directly on the radial sampled data, a more sophisticated algorithm for image reconstruction needs to be applied as the sampled data points on each radial spoke are not located on the grids as Cartesian sampling. The first issue is solved by the improvement of modern MR hardware, and the correction of predictable gradient delays [22]. The second issue is overcomed by properly interpolating the data points onto neighboring grid positions before the FFT reconstruction, and more details are discussed in the next section.

The key advantages of radial sampling scheme are that it is more robust to motion artifacts and tolerance against data undersampling. To further increase the temporal resolution of radial FLASH sequence, a sliding window technique is applied for data acquisition to reconstruct images from highly undersampled k-space data [21, 23, 24]. With the application of such a view sharing method, one can monitor the cardiac function with a temporal resolution of 50 frames per second. 


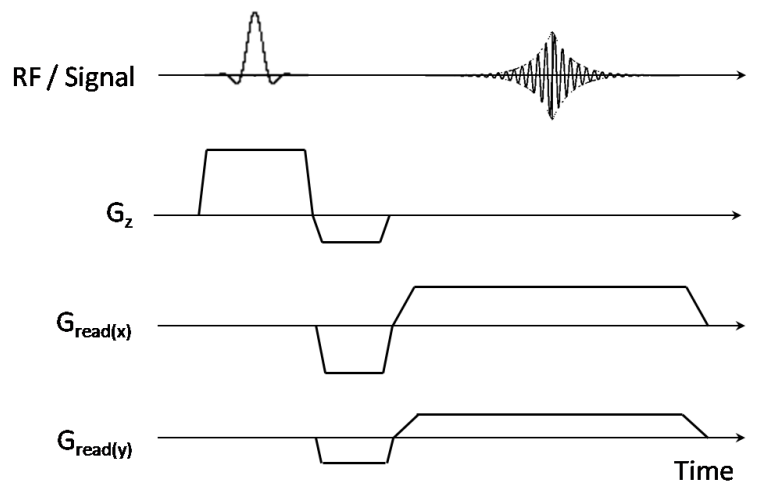

(a)

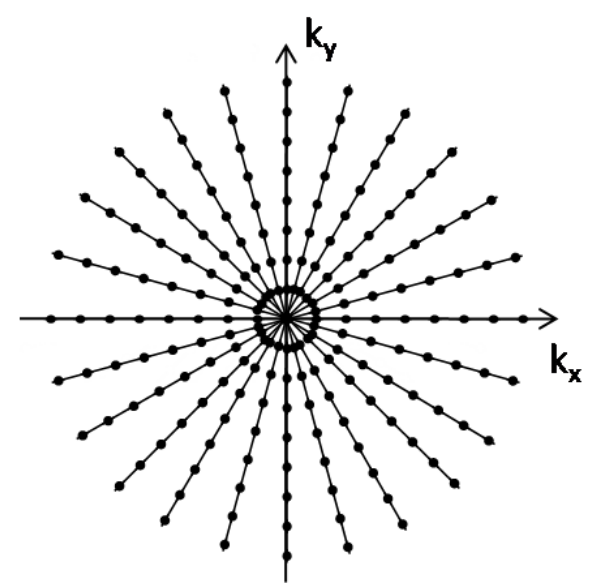

(b)

Figure 2.5.: (a) Sequence timing diagram of a 2D radial FLASH acquisition. (b) The corresponding sampling trajectory.

\subsubsection{Data Undersampling \& Nonlinear Inverse Reconstruction}

\section{Gridding Reconstruction}

For non-Cartesian schemes, such as radial encoding, FFT can not be performed directly on the acquired raw data for image reconstruction, because the collected raw data points are not necessarily on the Cartesian grids. Therefore, the data points need to be interpolated onto the neighboring Cartesian grids before performing FFT, which is called gridding [25-28] or non-uniform FFT (NUFFT) [29].

Generally, in order to perform gridding on radial sampled data sets, three steps are involved. Firstly, as shown in Figure 2.5(b), the radially sampled data points in the central $\mathrm{k}$-space are more condensed than that in the outer k-space. Therefore, a proper density compensation is required to weight every data point properly. Secondly, the weighted data needs to be equally interpolated onto the Cartesian grids before perform FFT. The Kaiser-Bessel window is considered as an optimal interpolation kernel, which is convolved with the k-space data as:

$$
K_{K B}(x)=\left\{\begin{aligned}
\frac{1}{L} I_{0}\left(\beta \sqrt{1-(2 x / L)^{2}}\right) & |x| \leq \frac{L}{2} \\
0 & |x|>\frac{L}{2}
\end{aligned}\right.
$$

where $L$ denotes the kernel width, $I_{0}$ is the zero-order modified Bessel function and $\beta$ is the shape factor. Furthermore, aliasing artifacts appear when applying FFT after data 
interpolation, because Equation (2.32) is a finite kernel which leads the side lobes outside of the desired image plane. This artifact is removed by oversampling the data points on each spoke and followed by a cropping operation after FFT.

\section{Parallel Imaging}

Modern MRI systems are equipped with multiple signal receiver coils for data acquisition. The simultaneous acquisition with multiple surface phase-array coils can not only reduce the image updating time but also improve the image SNR [30]. As a consequence, different from Equation (2.25), the actual acquired signal of each coil can be considered as the image content $\rho$ weighted by the corresponding coil sensitivity $c_{j}$ :

$$
s_{j}(t)=\int \rho(\vec{x}) \cdot c_{j}(\vec{x}) \cdot e^{-i 2 \pi \vec{k}(t) \vec{x}} d \vec{x}
$$

where $s_{j}(t)$ is the acquired raw data of the $j^{\text {th }}$ coil. The final anatomical image is usually carried out by applying FFT reconstruction on the k-space data of each coil and followed with a root of sum of squares (RSS) of all the coils.

According to Nyquist criterion for radial sampled trajectory, fully sampled k-space data has to fulfill the following relationship:

$$
n_{\text {spoke }}=\frac{\pi}{2} \cdot n
$$

where $n_{\text {spoke }}$ denotes the total number of spokes, and $\mathrm{n}$ is the desired matrix size. For instance, in order to reconstruct an image with a matrix size of $256 \times 256,402$ spokes are required so that the image does not suffer from streaking artifacts which is the typical artifact of undersampled radial trajectory. However, fully sampled k-space data leads to a temporal resolution of around one second, which is too slow to observe dynamic physiological motion, such as a beating heart.

A reasonable approach to accelerate the scanning time is to undersample the data in $\mathrm{k}$-space. According to Equation (2.33), if the coil sensitivity maps can be calculated or estimated, an artifact free image could be produced as the coil profiles provide additional spatial information that compensate the missing k-space data. Many studies consider Equation (2.33) as an inverse problem that estimates the image content and/or the coil sensitivities from the undersampled raw data and the k-space trajectory. The corresponding forward operator can be mathematically represented as: 


$$
F: x \mapsto\left(\begin{array}{c}
P \mathcal{F}\left\{\rho \cdot c_{1}\right\} \\
\vdots \\
P \mathcal{F}\left\{\rho \cdot c_{N}\right\}
\end{array}\right) \text {, with } x=\left(\begin{array}{c}
\rho \\
c_{1} \\
\vdots \\
c_{N}
\end{array}\right)
$$

where $x$ denotes all the unknown parameter maps, specifically image content $(\rho)$ and coil profiles $\left(c_{j}\right), \mathcal{F}$ is FFT and $P$ projects the data onto the trajectory.

Many algorithms have been proposed to eliminate artifacts due to data undersampling. Generally, based on the working domain, such approaches can be divided into two categories. For the first kind, reconstructions are performed in the Fourier domain, such as SMASH [31] and GRAPPA [32], which assume that the skipped k-space data are correlated with the neighboring sampled k-space data and can be extrapolated from the existing information with proper weights. For the second kind, reconstructions could also be performed in the image domain, such as SENSE [33, 34]. Coil sensitivities are calculated by a calibration scan or only fully sample data of k-space center. As a consequence, Equation (2.33) can be treated as a linear inverse problem and commonly solved by conjugate gradient $(\mathrm{CG})$ algorithm. However, the use of algorithm mentioned above could only achieve small acceleration factors. In the next section, a more complicated algorithm employed as the image reconstruction method for real-time MRI is introduced.

\section{Nonlinear Inverse Reconstruction}

Nonlinear inverse (NLINV) reconstruction, which updates the image $(\rho)$ and the coil information $\left(c_{j}\right)$ for each data acquisition to improve the MR temporal resolution, was first proposed for Cartesian sampled data [35, 36] and was later extended for radial sampling pattern $[1,37]$.

To estimate all the unknown parameters simultaneously, Equation (2.33) becomes a nonlinear system. NLINV employs the Iteratively Regularized Gauss Newton Method (IRGNM) to linearize the problem, presented as:

$$
\mathrm{y}=D F\left(\mathrm{x}_{n}\right) d \mathrm{x}+F\left(\mathrm{x}_{n}\right)
$$

where $\mathrm{x}_{n}$ denotes the estimation of the current Newton step, $D F\left(\mathrm{x}_{n}\right)$ represents the Fréchet derivative of the forward operator $F, d \mathrm{x}$ is the new update as $\mathrm{x}_{n+1}=\mathrm{x}_{n}+d \mathrm{x}$ and $\mathrm{y}$ is the MRI signal. Equation (2.36) can be considered as a reformulated quadratic optimization problem, which leads the cost function to:

$$
\Phi(d \mathrm{x})=\operatorname{argmin}\left\|D F\left(\mathrm{x}_{n}\right) d \mathrm{x}-\left(\mathrm{y}-F\left(\mathrm{x}_{n}\right)\right)\right\|_{2}^{2}+\alpha\left\|\mathrm{x}_{n}+d \mathrm{x}-\mathrm{x}_{0}\right\|_{2}^{2}
$$


with $\mathrm{x}_{0}$ being the initial guess which is weighted by a damping factor $(p, 0 \leq p \leq 1)$ with the estimation from the previous frame. On the right hand side of Equation (2.37), the first term represents the data consistency, the second term is the temporal regularization which is a conventional $l_{2}$ norm. The update rule of $d \mathrm{x}$ is:

$$
d \mathrm{x}=\left[D F\left(\mathrm{x}_{n}\right)^{H} D F\left(\mathrm{x}_{n}\right)+\alpha I\right]^{-1}\left[D F\left(\mathrm{x}_{n}\right)^{H}\left(\mathrm{y}-F\left(\mathrm{x}_{n}\right)\right)+\alpha\left(\mathrm{x}_{0}-\mathrm{x}_{n}\right)\right]
$$

with $D F(\mathrm{x})$ and $D F^{H}$ being the derivative and the adjoint derivative of $F(\mathrm{x})$, respectively.

Applying the IRGNM algorithm directly to solve the nonlinear inverse equation might yield undesired parameter maps, because the information of either coil sensitivities or the object could be unfolded inaccurately into each other without changing the result of $\rho \cdot c_{j}$. Such an ill-condition problem is overcome by taking a ground truth precondition knowledge into account, i.e. the coil profiles are rather smooth. With the application of the preconditioning matrix, the actual estimated parameters $(\hat{\mathrm{x}})$ become:

$$
\hat{\mathrm{x}}=\left(\begin{array}{c}
\rho \\
\hat{c_{1}} \\
\vdots \\
\hat{c_{N}}
\end{array}\right)=\left(\begin{array}{cccc}
I & & & \\
& \left(1+s\|\vec{k}\|^{2}\right)^{-l} \mathcal{F} & & \\
& & \ddots & \\
& & & \left(1+s\|\vec{k}\|^{2}\right)^{-l} \mathcal{F}
\end{array}\right)\left(\begin{array}{c}
\rho \\
c_{1} \\
\vdots \\
c_{N}
\end{array}\right)
$$

where $\vec{k}$ is Cartesian grids, practically $s=440$ and $l=16$ are used for algorithm implementation. In addition, in order to reduce the reconstruction time of NLINV method, multi graphical processing units (GPU), which communicate with the MRI scanner directly, are employed to provide on-line images [38].

With the combination of radial FLASH sequence and NLINV reconstruction algorithm, tens of images can be produced within one second. Real-time MRI technique has been extended into many clinical applications, such as cardiac function, dynamic joint studies and swallowing [39], speaking [40], cardiac flow [41] and tissue characterization [42]. Therefore, real-time MRI has a huge potential for dynamic temperature mapping during tissue thermal therapy. 



\section{Laser Induced Thermal Therapy and MR Thermometry Methods}

As an alternative of some conventional surgeries, nowadays routine clinical practice usually employs minimally invasive surgeries (e.g. laser induced thermal therapy (LITT) [43] and radio frequency (RF) ablation [44]) or noninvasive surgeries(high intensity focused ultrasound (HIFU) treatment [45]) on the target tissue to cure cancer. In the present work, a laser system is applied to generate a focal heating area and its working principle is introduced in this chapter. (More information can be found in [46].) Moreover, physical principles of MR temperature sensitive parameters are also reviewed in the later part.

\subsection{Laser Induced Thermal Therapy}

\subsubsection{Laser}

Laser is an acronym of 'light amplification by stimulated emission of radiation'. The theoretical foundation of laser has been described by A. Einstein in the early 1910's that electrons, which are excited into further orbits away from the nucleus of an atom, are prone to decay to their original state accompanied with emitting photons. This physical phenomenon was first discovered by C. Townes and A. Schawlow in 1958 [47], whose work was honored by Nobel Prize in 1964 and 1981, respectively.

Generally, a laser system consists of at least three components: a gain medium allows for stimulated emission, a pumping source provides energy into lasing medium, and a resonator adjusts laser output power, wavelength and direction. Compared with the ordinary light, laser enjoys many unique properties, such as high level of monochromaticity, coherence, directionality and brightness. Therefore, laser has a great number of applications in the field of science, medicine, military, industry and commerce.

\section{Semiconductor Lasers}

According to the active medium, laser can be characterized as gas, liquid or solid-state laser. A semiconductor laser system, which belongs to solid-state laser, is employed in the present work. 
The working principle of the semiconductor lasers is demonstrated in Figure 3.1. Generally, materials such as GaAs and InAs are used in the semiconductor laser. Due to the theory of electronic band structures, in those materials, the valence band and the conduction band are the highest and the lowest energy states of a vacant electronic, respectively and the two bands are separated by the band gap. As shown in Figure 3.1 (a), the valence band is fully occupied by electrons (in the shadow region), whereas the conduction band is completely free of electrons. Subsequently, assuming that a number of electrons are excited by proper external energy to the conduction band (Figure 3.1 (b)), the energy of electrons in the valence and the conduction band are expressed as $E_{F v}^{\prime}$ and $E_{F c}^{\prime}$ respectively. With the electrons fall back to the valence band, light emission also occurs. Note that the excitation energy and the emission energy of an electron fulfill the following relationship:

$$
E_{g} \leq h \nu \leq E_{F c}^{\prime}-E_{F v}^{\prime}
$$

where $E_{g}$ is the energy gap between the two bands, $h \nu$ is the electron transition energy with $h$ being the Planck's constant and $\nu$ is the emission frequency.

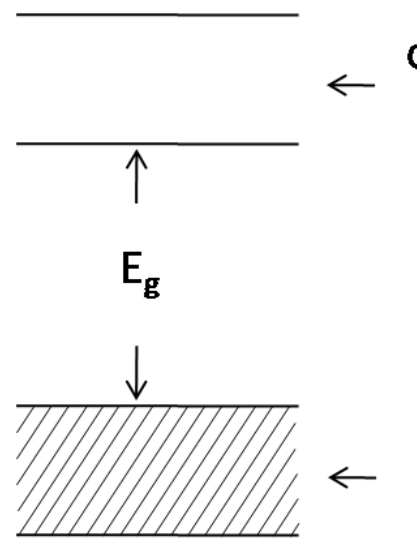

(a)

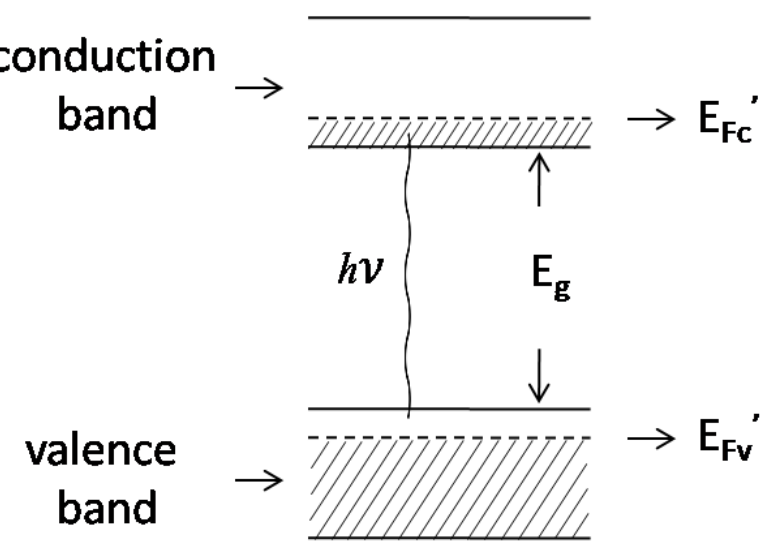

(b)

Figure 3.1.: The working principle of the semiconductor laser.

\section{Laser System}

As shown in Figure 3.2, a commercial semiconductor laser system (RevoLix jr.30, Lisa Laser, Katlenburg-Lindau, Germany) is employed in the present work for many experiments to create a focal heating area and it can produce continuous, pulsed and single pulse laser energy. The system is located outside the MR scanner room and its energy is introduced into the target tissue via a ten-meters fiber. More technical details of the 
laser system can be found in table 3.1.

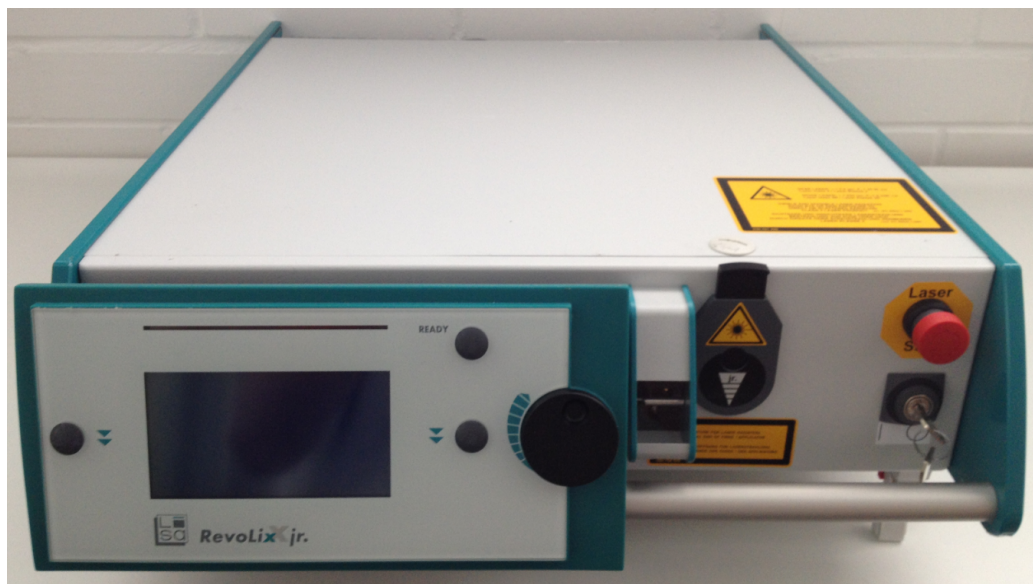

(a)

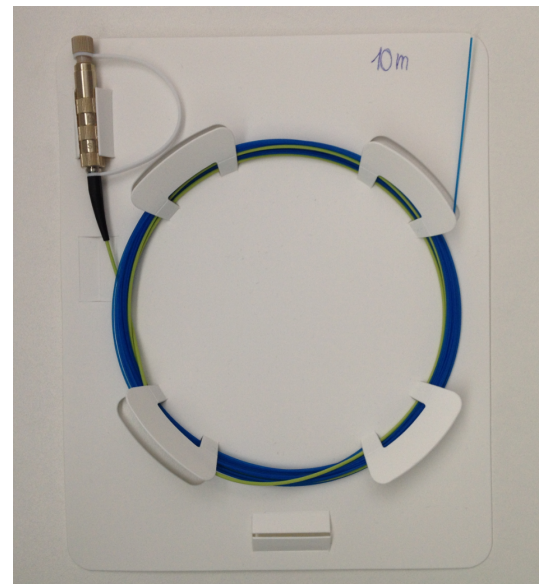

(b)

Figure 3.2.: (a) The laser generator. (b) The laser fiber.

Table 3.1.: Technical parameters of laser system.

\begin{tabular}{cc}
\hline \multicolumn{2}{c}{ RevoLix jr.30 Laser } \\
\hline Wavelength & $\approx 2 \mu \mathrm{m}$ \\
Penetration depth & $\approx 1 \mathrm{~mm}$ \\
Output power & $1 \sim 30 \mathrm{w}$ \\
Pulse duration & $20 \sim 1000 \mathrm{~ms}$ \\
Repetition rate & $0.5 \sim 25 \mathrm{~Hz}$ \\
\hline \multicolumn{2}{c}{ Fiber } \\
\hline Length & $10 \mathrm{~m}$ \\
Outer diameter & $0.75 \mathrm{~mm}$
\end{tabular}

\subsubsection{Heat Transfer in Tissue}

Generally, thermal therapy is categorized into two types. The first kind is hyperthermia, which damages the cancer tissue by increasing the temperature of the target region to $43-45^{\circ} \mathrm{C}$ in tens of minutes [48]. While the other kind, tissue coagulation, kills the tumor only in a few minutes by increasing the temperature of the target tissue to $50-80{ }^{\circ} \mathrm{C}$ [49]. Table 3.2 shows an overview of tissue interactions under different temperature range $[50,51]$. The present work pays more attention on tissue coagulation experiments, because real-time MRI is more suitable for monitoring rapid surgery procedures. 
Table 3.2.: Tissue interaction under different temperature ranges.

\begin{tabular}{cc}
\hline Temperature Range $\left({ }^{\circ} \mathrm{C}\right)$ & Tissue Interaction \\
\hline $35-40$ & Normothermia \\
$42-46$ & Hyperthermia \\
$46-48$ & Irreversible cellular damage at $45 \mathrm{~min}$ \\
$50-52$ & Coagulation necrosis at $4-6$ min \\
$60-100$ & Instantaneous coagulation necrosis \\
110 & Tissue vaporization \\
\hline
\end{tabular}

To have a deep understanding of temperature distribution during thermal therapy, $\mathrm{H}$. Pennes [52] proposed a so-called bio-heat transfer (BHT) model, expressed as:

$$
\rho_{t} c_{t} \frac{\partial T(\vec{r}, t)}{\partial t}=\nabla \cdot k \nabla T(\vec{r}, t)-w_{b} \rho_{b} c_{b}\left[T(\vec{r}, t)-T_{a}\right]+p(\vec{r})
$$

where $\rho_{t}$ and $\rho_{b}\left(\mathrm{~kg} / \mathrm{m}^{3}\right)$ represent the tissue and blood density, $c_{t}$ and $c_{b}\left(\mathrm{~J} / \mathrm{kg} /{ }^{\circ} \mathrm{C}\right)$ are the specific heat of tissue and blood, $T(\vec{r}, t)$ and $T_{a}\left({ }^{\circ} \mathrm{C}\right)$ are the temperature of local tissue and arterial blood, respectively, $k_{t}\left(\mathrm{~W} / \mathrm{m} /{ }^{\circ} \mathrm{C}\right)$ is the tissue thermal conductivity, $w_{b}\left(\mathrm{~kg} / \mathrm{m}^{3} / \mathrm{s}\right)$ represents the tissue-blood perfusion rate and $p\left(\mathrm{~W} / \mathrm{m}^{3}\right)$ can be understood as a constant power density absorbed by the local tissue. Equation (3.2) holds true under two assumptions: a) the tissue of interest should be absent from large blood vessels, b) blood in the capillaries enters the heated region at the body temperature and moves out at the local temperature. Assuming that the local tissue and blood share the same density and specific heat $\left(\rho_{t}=\rho_{b}, c_{t}=c_{b}\right)$, Equation (3.2) can be simplified as:

$$
\frac{\partial \hat{T}(\vec{r}, t)}{\partial t}=D \nabla^{2} \hat{T}(\vec{r}, t)-w_{b} \hat{T}(\vec{r}, t)+\alpha p(\vec{r})
$$

with $\hat{T}=T-T_{a}, \alpha=1 /\left(\rho_{t} c_{t}\right), \mathrm{D}\left(\mathrm{m}^{2} / \mathrm{s}\right)$ representing the thermal diffusivity and $\nabla^{2}$ being the Laplacian operator. Similar to Equation (3.3), the temporal-spatial temperature evolution without thermal deposition can be written as:

$$
\frac{\partial \hat{T}(\vec{r}, t)}{\partial t}=D \nabla^{2} \hat{T}(\vec{r}, t)-w_{b} \hat{T}(\vec{r}, t)
$$

The BTH model demonstrates that the spatial distribution of temperature depends on the thermal diffusivity, and the tissue perfusion rate can be considered as the scaling factor of temperature changes [53]. In the present work, non-perfused ev vivo tissues are used as the experimental materials. A more comprehensive analysis with respect 
to Equation (3.3) and (3.4) can be found in appendix A. More details are referred to $[50,53,54]$.

\subsection{MR Thermometry Methods}

Different temperature sensitive MR parameters represent different MR temperature mapping methods, such as proton resonance frequency (PRF), $T_{1}$ relaxation time, diffusion coefficient, proton density and so on. The relationships between temperature and those parameters are demonstrated in this section. More attentions are paid on PRF and $T_{1}$ relaxation time methods, due to their high temperature sensitivities and rapid data acquisition schemes.

\subsubsection{Proton Resonance Frequency}

For a signal proton, as shown in Equation (2.8), the corresponding Larmor frequency is proportional to the superposition of the external magnetic field $\left(B_{\text {ext }}\right)$ and the screening effect by the free electron cloud, written as:

$$
\omega=\gamma(1-\sigma) B_{\text {ext }}
$$

with $\sigma$ being the screening factor. In 1966, J.C Hindman discovered that the screening effect is enhanced with the increase of temperature [55]. To be more specific, in liquid phase water, free protons provide screening effect with respect to the external magnetic field. Due to the fact that many protons in the neighboring water molecules are connected in the form of hydrogen bonds, the total number of free protons decreases leading to a reduction of the screen effect. By increasing the temperature, the fraction of hydrogen bonds releases more free protons and thus the shielding becomes more effective which results in a lower proton resonance frequency. Therefore, Equation (3.5) can be rewritten as:

$$
\omega=\gamma(1-\sigma(T)) B_{\text {ext }}
$$

Hindman also reported that $\Delta \sigma(T)$ is proportional to the temperature changes (from - 15 to $100{ }^{\circ} \mathrm{C}$ ), expressed as:

$$
\Delta \sigma(T)=\alpha \Delta T
$$

with a temperature coefficient $\alpha=-0.01 \mathrm{ppm} /{ }^{\circ} \mathrm{C}[55-57]$, which is adapted for all the 
experiments in the present work. Based on Equation (3.7), at a given TE, the phase contrast map can be derived as:

$$
\Delta \phi=\gamma\left[\sigma(T)-\sigma\left(T_{0}\right)\right] B_{e x t} \mathrm{TE}
$$

where $T$ is the current temperature and $T_{0}$ represents the reference temperature. Substituting Equation (3.7) into (3.8) yields:

$$
\Delta T=T-T_{0}=\frac{\Delta \phi}{\alpha \gamma B_{\text {ext }} \mathrm{TE}}
$$

In this study, $\gamma B_{\text {ext }}$ equals to $123.251 \mathrm{MHz}$, which is confirmed from the used MRI system in this work. Note that PRF method generates relative temperature differences, therefore, a reference data either at the body (in vivo study) or the room temperature (ex vivo study) is required for the calculation.

The PRF method was first employed in MR thermometry by Y. Ishihara [58] and J. De Poorter [59, 60] and it has been proved to be a tissue type independent mechanism [61] (except for adipose tissue) for temperature mapping. Compared with aqueous tissue, the PRF shifts with temperature in lipid could be neglected $\left(\alpha_{f a t}=0.00019 \mathrm{ppm} /{ }^{\circ} \mathrm{C}\right)$ [62], because MR signal of fat is supplied by methylene $\left(\mathrm{CH}_{2}\right)_{n}$, rather than hydrogen bond filled with water proton $[63,64]$.

\subsection{2. $\mathrm{T}_{1}$ Relaxation Time}

$T_{1}$, the longitudinal relaxation time, is also dependent on the temperature changes and it was first reported by N. Bloembergen [65]. In biological tissues, spin lattice relaxation $\left(T_{1}\right)$ depends on the dipolar interaction between macromolecules and water molecules in different compartment (free and bound state). The translational and the rotational motion of free state water molecules dominate such an interaction process and they are effected by temperature. Previous studies have already proven that the relationship between temperature and $T_{1}$ can be expressed as [66-68]:

$$
T_{1} \propto e^{\left(-E_{a} / k T\right)}
$$

with $E_{a}$ being the activation energy during relaxation process, $k$ being the Boltzmann constant and $T$ representing the absolute temperature. The derivative of Equation (3.10) on temperature is:

$$
\frac{d T_{1}}{d T}=\frac{E_{a} T_{1}}{k T^{2}}
$$


Substituting $d T_{1}=T_{1}-T_{1, \text { ref }}$ and $d T=T-T_{\text {ref }}$ into Equation (3.11), with $T_{1}$ and $T_{1, \text { ref }}$ being the longitudinal relaxation time under current $(T)$ and baseline temperature $\left(T_{r e f}\right)$, respectively, leads to the following expression:

$$
\Delta T=\frac{k T_{r e f}^{2}}{E_{a}}\left(\frac{\Delta T_{1}}{T_{1, r e f}}\right)
$$

Consequently, within a small temperature range, $T_{1}$ linearly changes with temperature, which can be written as:

$$
T_{1}=T_{1, r e f}+\beta\left(T-T_{r e f}\right)
$$

where $\beta$ is a tissue type dependent constant which needs to be determined empirically. Temperature dependency $(\beta)$ on $T_{1}$ is experimentally confirmed about at $1 \% /{ }^{\circ} \mathrm{C}[64,69-$ 71]. $T_{1}$ MR thermometry has been successfully performed on both ex vivo as well as in vivo tissues during the past two decades [72-74].

\subsubsection{Alternative Temperature-sensitive MR Parameters}

Many other MR parameters are also discovered to be sensitive to temperature, such as magnetization $\left(M_{0}\right)$, diffusion coefficient $(D)$ and transversal relaxation time $\left(T_{2}\right)$. However, they all show considerable practical limitations for clinical applications. A further overview of all the available methods can be found in [75-77].

\section{Proton Density}

According to Equation (2.7), the thermal equilibrium magnetization $\left(M_{0}\right)$ is proportional to the proton density $(\rho)$ and can be expressed as:

$$
M_{0}=\rho \frac{\gamma^{2} \hbar^{2}}{4 k T} B_{0}
$$

Equation (3.14) indicates that $M_{0}$ is inverse proportional to the absolute temperature with a sensitivity of $-0.3 \% /{ }^{\circ} \mathrm{C}[78]$. The drawbacks of this physical parameter are: on one hand, long TR is required for obtaining pure proton density weighted images which leads to a long data acquisition; on the other hand, compared with other methods, its temperature dependency is rather small $\left(-0.3 \% /{ }^{\circ} \mathrm{C}\right)$. 


\section{Diffusion Coefficient}

The Brownian motion of molecules in human tissues is described as diffusion coefficient $(D)$ and its relationship with temperature is written as:

$$
D \approx e^{-E_{a}(D) / k T}
$$

with $E_{a}(D)$ being the activation energy of diffusion of water molecules, $k$ being the Boltzmann constant, $T$ representing the absolute temperature. The temperature sensitivity of diffusion is about $2 \% /{ }^{\circ} \mathrm{C}[79,80]$. A major disadvantage of applying diffusion in in vivo temperature mapping is that diffusion coefficient varies dramatically when the target tissue is coagulated under high temperature.

\section{$\mathrm{T}_{2}$ Relaxation Time}

Similar to $T_{1}$, spin-spin relaxation time $\left(T_{2}\right)$ also shows a temperature dependency. However, $T_{2}$ in biological tissue is normally much shorter than that in pure water, which might be effected by the interactions between water molecules in tissue. Thus, $T_{2}$ has a lower temperature sensitivity [68] and is not suitable for temperature mapping.

In conclusion, compared with other parameters, both PRF and $T_{1}$ enjoy the merits of high temperature sensitivities and the possibility of fast data acquisition scheme. Therefore, in this thesis, these two methods will be studied and compared comprehensively for real-time MR temperature mapping. 


\section{Real-time MR Thermometry - Phantom Studies}

In order to quantitatively evaluate and compare different temperature mapping methods, phantom studies play an important role. Therefore, in this chapter, the construction of a home made phantom applicable for temperature mapping is introduced. For the second part, based on the phantom, methodologies and results of PRF and $T_{1}$ MR thermometry are demonstrated.

\subsection{Phantom Development}

To mimic tissue thermal therapy, an appropriate phantom must fulfill the following properties: a) MR compatibility, b) resistance to heat, c) equivalent $T_{1}$ value as in human tissue, d) the ability to mimic tissue coagulation procedure. Several versions of phantoms have been developed and tested, and the final design is shown in Figure 4.1.

The phantom (Figure 4.1) consists of three components: a central tube is connected with a pump (ED(v.2), JULABO GmbG, Seelbach, Germany) that provides high temperature water $\left(50{ }^{\circ} \mathrm{C}\right)$ with constant flow; a cylinder container is filled with doped agarose; a outer container is connected with another pump (FL601, JULABO GmbG, Seelbach, Germany) that offers low temperature water $\left(22{ }^{\circ} \mathrm{C}\right)$ with constant flow. Under the influences of both the heating and the cooling systems, a temperature gradient can be generated within the middle cylinder. In order to record the temperature changes, three fiber optic temperature sensors (Figure 4.1 (b)) (OPT-M, OPSENS, Quebec, Canada) are inserted into the agarose parallel to the central tube. The tips of sensors are kept in the same line at a radius of 1, 2, $3 \mathrm{~cm}$ to the central tube. Moreover, four small reference phantoms (indicated in Figure 4.1 (c)) are used to compensate the field drift for PRF method (more details seen in chapter 4.2) and equally positioned surrounding the main phantom. The cylinder container and the reference tubes are filled with doped agarose, which is often considered as a tissue equivalent material for MRI studies [81-84]. In the present work, $2 \%$ agarose with $0.5 \mathrm{mMol} / \mathrm{L} \mathrm{CuSO}_{4}$ is used as the filling material, which has a $T_{1}$ value of about $1000 \mathrm{~ms}$ at room temperature $[81,82]$ 
After systematic tests, the phantom can be heated from room temperature to at least $36{ }^{\circ} \mathrm{C}$, and generate a temperature gradient of at least $8{ }^{\circ} \mathrm{C}$ from the location of fiber optic sensor 1 to 3. Moreover, as demonstrated in Figure 4.2, the phantom reaches a new thermal equilibrium after about two hours. Due to the different relative distances to the central tube, a quick temperature increase is observe with fiber optic sensor 1, and delayed increases are recorded with fiber optic sensor 2 and 3.

\subsection{PRF Thermometry}

\subsubsection{Methods and Materials}

\section{Sequence Design and Optimization}

RF spoiled radial FLASH is used for data acquisition, which applies continuous shifted RF pulses to prevent the transversal magnetization from reaching steady state and does not require additional gradients to achieve a higher temporal resolution [85, 86]. A spin echo based sequence can not be employed for PRF thermometry, because the temperature dependent phase information will be refocused during data acquisition. A large number of sequence parameters, which can influence the quality of the anatomical images and temperature maps, are discussed and evaluated in this section.

Flow Compensation Gradient: Once the pumps for heating and cooling connected with the phantom are switched on, the water from the two pumps can be considered with a constant velocity. In order to eliminate the phase artifacts induced by the flowing spins, a flow compensation (or motion compensation) gradient is employed for data acquisition. As illustrated in Figure 4.3, for both static and moving spins, the accumulated phase is zero at the end of the gradient $[87,88]$. Figure 4.4 shows the magnitude images acquired without and with the flow compensation gradient implemented in the sequence. The image acquired with the flow compensation has an increased signal intensity in the areas indicated by the red arrows. However, turbulent flow is still observed in the outer container (shown by the white arrows), because the applied motion compensation gradient only gains zero net phase for the spins with constant velocity.

Echo Time and Bandwidth: The relationship between TE and SNR with respect to phase difference is described as:

$$
\mathrm{SNR}_{\Delta \phi} \propto \mathrm{TE} e^{-\mathrm{TE} / T_{2}^{*}}
$$

with $T_{2}^{*}$ being the effective magnetization decay in the transversal plane. According to 


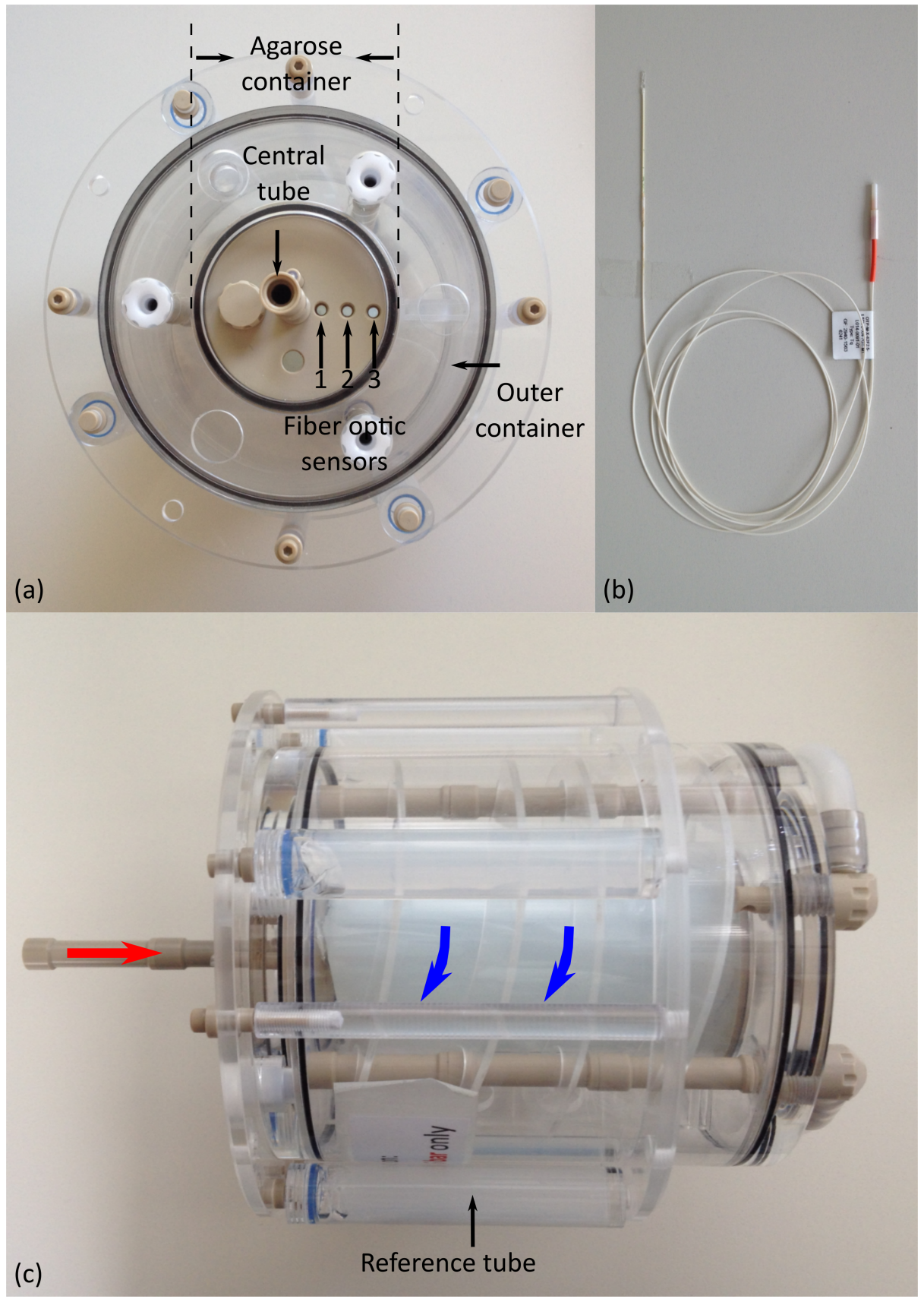

Figure 4.1.: Home made temperature phantom. (a) Front view of the phantom. The inner container is filled with doped agarose. Three fiber optic sensors are inserted into the phantom to record the temperature changes. The central tube and the outer container are connected with a cycled warm $\left(50^{\circ} \mathrm{C}\right)$ or cold $\left(22^{\circ} \mathrm{C}\right)$ water system, respectively. (b) A fiber optic sensor used for temperature recording. (c) Side view of the phantom. Red and blue arrows represent the flow direction of the warm and cold water, respectively. One of the four reference tubes is indicated. 


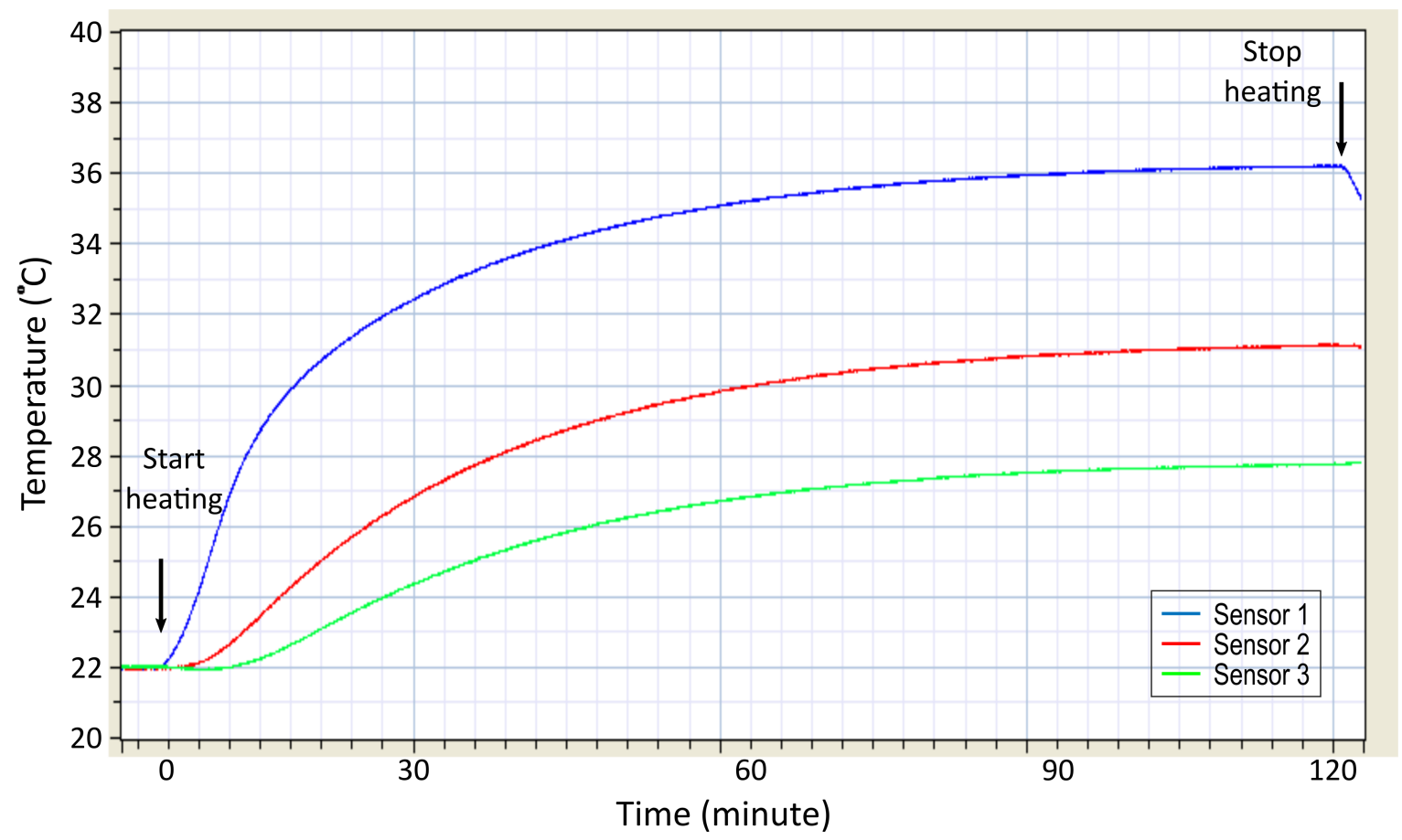

Figure 4.2.: Temperature recorded by the three fiber optic sensors during two hours of heating. The experiment is performed outside the magnet in the MR scanner room. The sensors are inserted into the phantom as shown in Figure 4.1 (a). The three curves demonstrate that the agarose container reaches a new thermal equilibrium after two hours.

Equation 4.1, in order to acquire phase contrast maps with high SNR, two issues need to be concerned. On one hand, the phase difference between the current processed and the reference datasets increases linearly with TE based on Equation (3.8). One the other hand, signal SNR exponentially decays with the increase of TE. Ideally, the optimal TE can be obtained by differentiating the equation above on TE, which is $\mathrm{TE}=T_{2}^{*}[86,89]$. However, the $T_{2}^{*}$ of kidney, which is often used as the ex vivo tissue material in later experiments, is around $25 \mathrm{~ms}$ at $3 \mathrm{~T}$ [90]. Two major disadvantages appear when a TE of $25 \mathrm{~ms}$ is performed in the PRF thermometry sequence. Firstly, base on Equation (3.9), the maximum detectable temperature rise in a $3 \mathrm{~T}$ MRI system is $\Delta \mathrm{T}=16{ }^{\circ} \mathrm{C}$ which is not sufficient for the tissue coagulation therapy (normally from body temperature $\left(37^{\circ} \mathrm{C}\right.$ ) to almost $60{ }^{\circ} \mathrm{C}$ ), a temperature rise above $16{ }^{\circ} \mathrm{C}$ leads to a phase wrapping problem and might be considered as an insufficient temperature increase in the target tissue. Secondly, anatomical images suffer from low SNR, due to the effects of $T_{2}^{*}$ decay and the data undersampling of real-time purpose. In the present work, TE is set as $10 \mathrm{~ms}$, which leads to a detectable maximum temperature increase of $39{ }^{\circ} \mathrm{C}$. The temperature accuracy is evaluated in this section.

In general, bandwidth (BW) of the receiver coil is a sequence parameter that balances the temporal resolution and the image SNR. An increase of BW can shorten the TE so that 


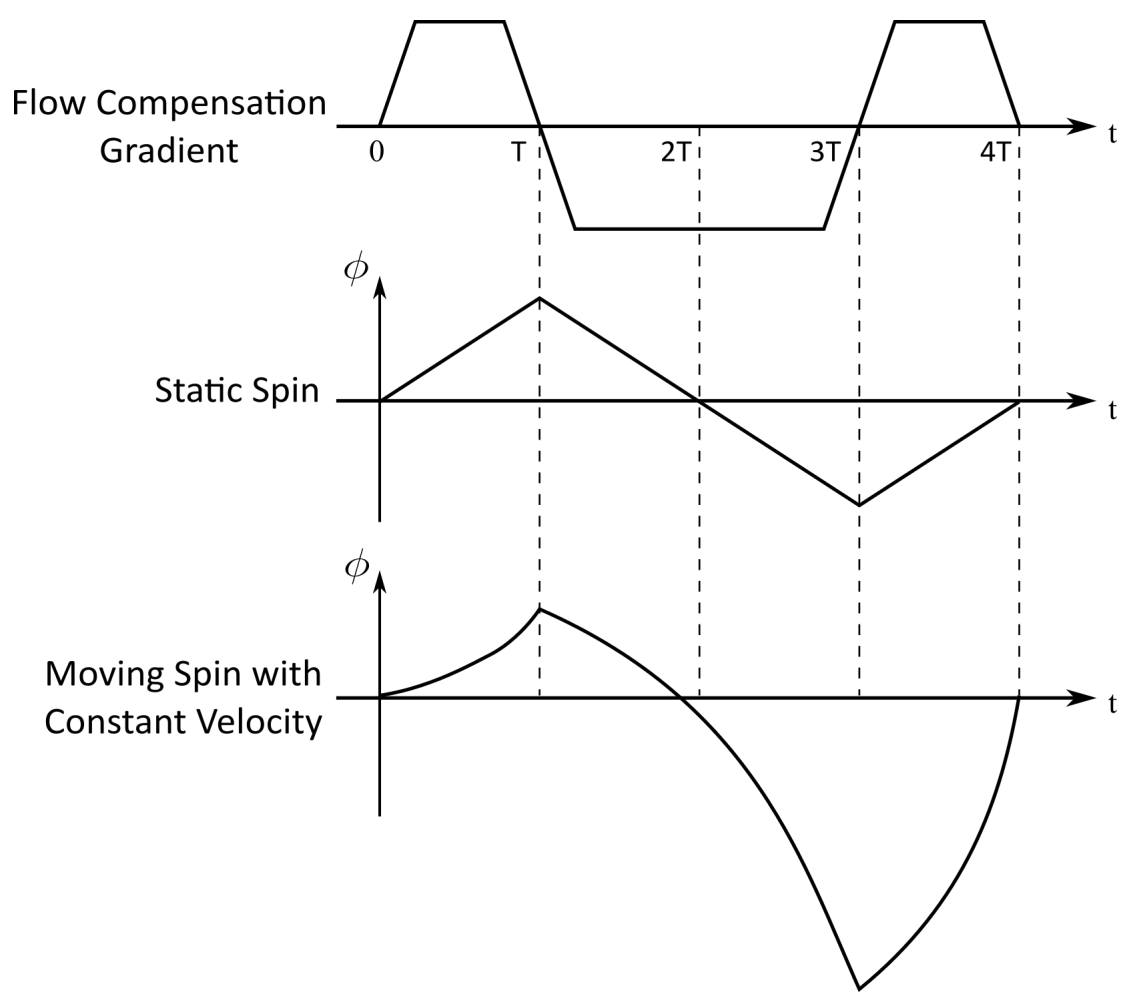

Figure 4.3.: (Top) Flow compensation gradient with $1 \overline{2} 1$ waveform. (Middle) Accumulated phase of a static spin shows a linear relationship with the duration of the flow compensation gradient. (Bottom) Accumulated phase of a moving spin with constant velocity shows a quadratic relationship with the duration of the flow compensation gradient.

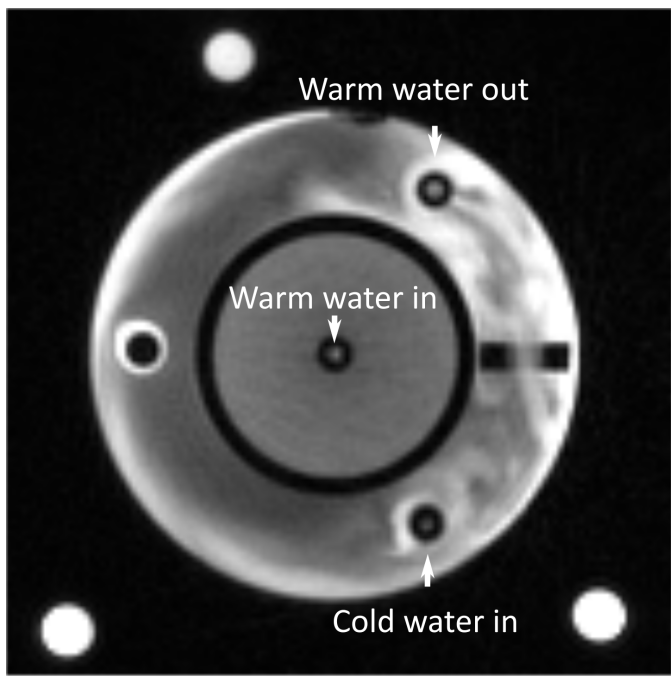

(a)

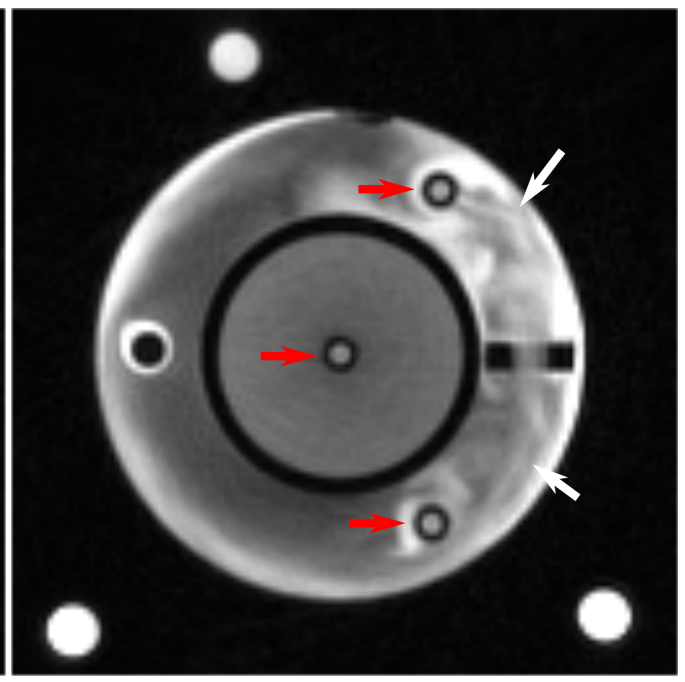

(b)

Figure 4.4.: (a) Magnitude images of the temperature phantom acquired without and (b) with a flow compensation gradient. The red arrows indicate the expected signal increase due to the application of the gradient. The white arrows indicate uncompensated turbulent flow. 
leads to a shorter TR and vice versa. However in the temperature mapping sequence, TE is already chosen as $10 \mathrm{~ms}$, in order to compensate the long TE induced SNR decrease, a relative small BW (compared with $T_{1}$ weighted radial FLASH sequence [42]) is used for data acquisition $(\mathrm{BW}=650 \mathrm{~Hz} /$ Pixel).

Other MR Parameters used for PRF thermometry of the temperature phantom are summarized in Table 4.1. A TR of $11.8 \mathrm{~ms}$ and 17 radial spokes lead to an image updating time of $200 \mathrm{~ms}$ for both anatomy and temperature images. This is fast enough to monitor tissue motion and temperature change [77]. The in-plane resolution is set to $1.5 \times 1.5 \mathrm{~mm}^{2}$ and the slice thickness is $6 \mathrm{~mm}$.

Table 4.1.: MR parameters used for PRF thermometry on the phantom.

\begin{tabular}{cc|cc}
\hline Sequence & Radial FLASH & FOV & $192 \times 192 \mathrm{~mm}^{2}$ \\
\hline TE/TR & $10 / 11.8 \mathrm{~ms}$ & In-plane resolution & $1.5 \times 1.5 \mathrm{~mm}^{2}$ \\
\hline Flip angle & $10^{\circ}$ & Number of spokes & 17 per frame \\
\hline Slice thickness & $6 \mathrm{~mm}$ & Temporal resolution & $200 \mathrm{~ms}$ \\
\hline
\end{tabular}

\section{Image Reconstruction}

NLINV Parameters: As introduced in Chapter 3, real-time MRI applies Gauss Newton method to joint estimate the image content and the corresponding coil sensitivity maps iteratively. According to Equation (2.37), two reconstruction parameters have a strong influence on the image quality, which are the number of Newton steps and the damping factor. A low number of Newton steps can generate blurring images and thus different tissue structure may not be distinguished properly. Conversely, too many iterations generate more noise in the image, because the data consistency term in Equation (2.37) dominates the reconstruction process in later Gauss Newton steps. Damping factor, which is involved in the temporal regularization term, can be varied from 0 to 1 and represents the similarity between the current and the previous image content. In the present work, in order to compute accurate phase contrast maps, 7 Newton steps and a damping factor of 0.7 are performed for image reconstructions.

Multi-channel Phase Contrast Mapping: As discussed in Chapter 2, the used MRI system is equipped with multi-receiver coils. In order to reduce the computation time of NLINV reconstruction, ten virtual coils are selected from the first frame by applying principal component analysis (PCA) method and are kept for the entire data stream acquired during the heating procedure. For image reconstruction, different from SENSE method [33, 34], NLINV updates the proton density and the coil profiles simultaneously 
for every frame. Therefore, all the coil sensitivity maps have to be taken into account for the phase difference mapping.

The reconstructed complex image of a single coil $\left(\vec{M}_{i}\right)$ can be calculated by multiplying the image content $(\vec{\rho})$ with the corresponding coil profile $\left(\vec{c}_{i}\right)$ :

$$
\begin{aligned}
\vec{M}_{0, i} & =\vec{\rho}_{0} \cdot \vec{c}_{0, i} \\
\vec{M}_{1, i} & =\vec{\rho}_{1} \cdot \vec{c}_{1, i}
\end{aligned}
$$

where the index 0 and 1 represent the baseline and the current processed temperature data, respectively. The phase difference map of a single coil $\left(\Delta \phi_{i}\right)$ can be calculated by the following equations:

$$
\begin{gathered}
\Delta \phi_{i}=\arg \left(\vec{M}_{1, i} \cdot \vec{M}_{0, i}^{*}\right) \\
\text { with: } \vec{M}_{1, i} \cdot \vec{M}_{0, i}^{*}=\left|\vec{M}_{1, i}\right| \cdot\left|\vec{M}_{0, i}\right| \cdot e^{i\left(\phi_{1}-\phi_{0}\right)}
\end{gathered}
$$

where $\phi_{1}$ and $\phi_{0}$ are the phase information of the reference and the current temperature, respectively and $*$ represents the complex conjugate operation on the matrix. The phase contrast map over all the receiver channels is expressed as [91]:

$$
\Delta \phi=\arg \left[\sum_{i=0}^{n}\left(\vec{M}_{1, i} \cdot \vec{M}_{0, i}^{*}\right)\right]
$$

with $n$ being the number of the receiver coils. Once the phase contrast maps are computed by the formulas above, based on Equation (3.9), temperature maps can also be calculated.

\section{Experimental Procedures}

A 64-channel head coil was employed for data acquisition. The temperature phantom was located in the head coil with the central tube parallel to the main magnetic field (longitudinal axis) and data acquired in the transverse plane were always employed for temperature calculations. Before each experiment, the phantom was placed in the scanner room for at least 8 hours to ensure thermal equilibrium. During the experiments, water with $50{ }^{\circ} \mathrm{C}$ and $22{ }^{\circ} \mathrm{C}$ (room temperature) were provided by the two pumps. For postprocessing, in order to remove streaking artifacts from data undersampling, median filter technique was performed on both the magnitude and temperature images [21]. Moreover, the phase information of the four reference tubes were used to correct the phase error caused by magnetic field drift [61]. 


\section{Real-time MR Thermometry - Phantom Studies}

Evaluation of PRF Method: In order to validate the accuracy of the PRF method, the reference data was collected before switching on the cycled water. When the phantom reached a new thermal equilibrium (two hours later, as demonstrated in Figure 4.2), the pumps were switched off and another data set was measured. Additionally, for each acquisition, two slices were sampled. The first slice contained the tips of temperature sensors, which record the true temperature (Figure 4.5 (a)), and the second is the neighboring slice, which was used for data calculation (Figure 4.5 (b)). Relative temperature difference maps were calculated based on Equation (4.4) and (3.9). Three regions of interest (ROI) were chosen in the second slice according to the location of the sensors for data validation (indicated in Figure 4.5 (d)).

Dynamic Temperature Mapping by PRF Method: For the purpose of monitoring temperature change in real-time, after acquiring reference images, the two pumps and the MR sequence were started simultaneously for half an hour. The movie of temperature increase was generated based on Equation (4.4) and (3.9).

\subsubsection{Results}

The results of the assessment of PRF thermometry are shown in Figure 4.5. Due to the fact that the reconstructed complex images only differ in phase between the baseline and the current temperature data, the corresponding magnitude images (Figure 4.5 (b) and (c)) are almost visually identical (both pumps are switched off). The calculated temperature difference map shows a symmetrical temperature gradient from inside to outside of the agarose container (Figure $4.5(\mathrm{~d})$ ). The temperature profile along a reference line (indicated by the dashed line in Figure $4.5(\mathrm{~d})$ ) is depicted in Figure 4.6. The temperature near the central tube is increased by around $19{ }^{\circ} \mathrm{C}$ and at the outer edge, an increase of more than $2{ }^{\circ} \mathrm{C}$ can be observed. In addition, the quantitative analysis is performed by using 3 ROIs in the agarose container according to the locations of the temperature sensors (Figure $4.5(\mathrm{a})$ and $(\mathrm{d})$ ). The mean value and the standard deviation (SD) of each ROI are summarized in Table 4.2 and show very good agreement with the recorded data from the sensors. All the temperature uncertainties indicated by SDs of the calculated data are below $10 \%$.

As for the dynamic monitoring experiment, half an hour data acquisition with a temporal resolution of $200 \mathrm{~ms} /$ frame yields 9000 frames in total. The magnitude images and the corresponding temperature maps for every five minutes are displayed in Figure 4.7. The development of the temperature gradient can be observed. In addition, the temperature changes of the three ROIs (indicated in Figure 4.7) are shown in Figure 4.8. As expected and similar to the results obtained with the fiber optic sensors, due to the different rel- 
ative distances to the central tube, a quick temperature increase is observe with ROI 1 and the temperature is increased by $6{ }^{\circ} \mathrm{C}$ at ten minutes and $9{ }^{\circ} \mathrm{C}$ at 30 minutes. Delayed increases are recorded with ROI 2 and 3, and the temperature is increased by $4{ }^{\circ} \mathrm{C}$ and $2{ }^{\circ} \mathrm{C}$ at 30 minutes, respectively. Because of the cycled cold water runs in a spiral way in the outer container, water turbulence is observed in the magnitude images.

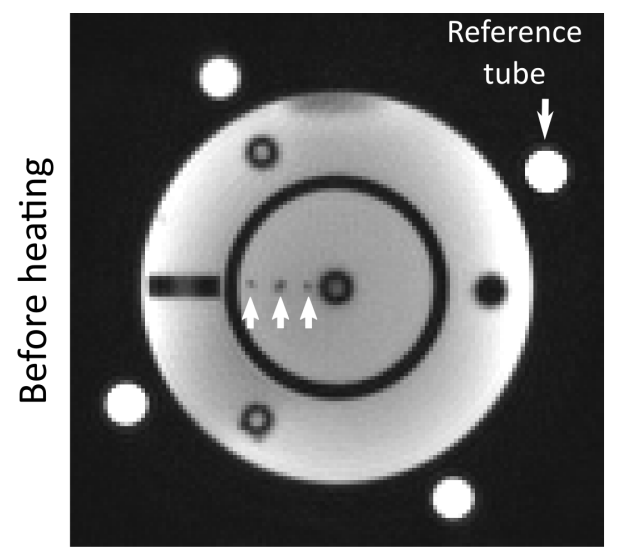

(a)

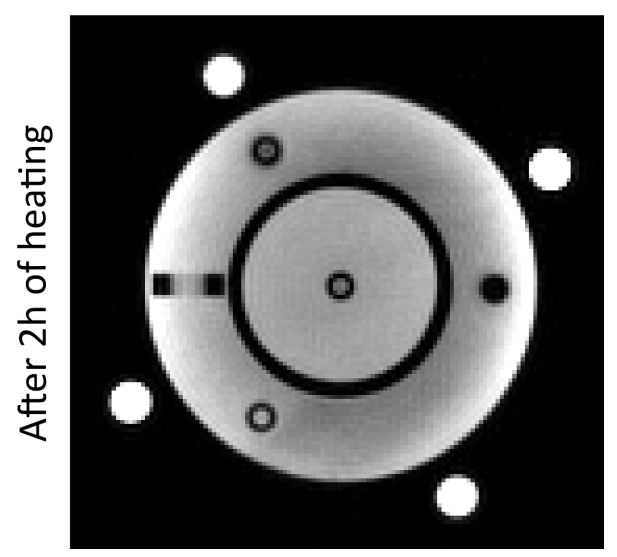

(c)

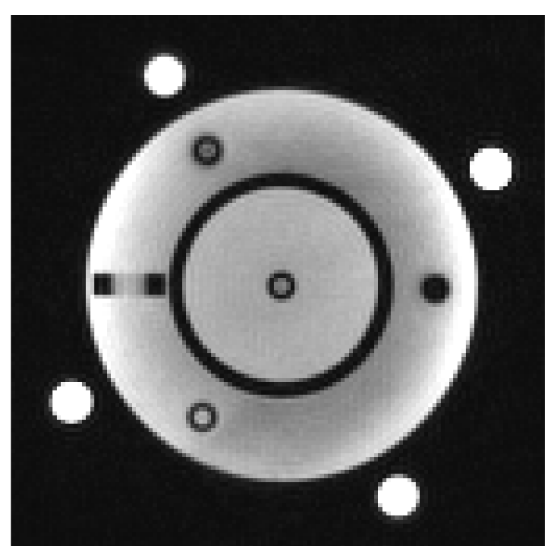

(b)

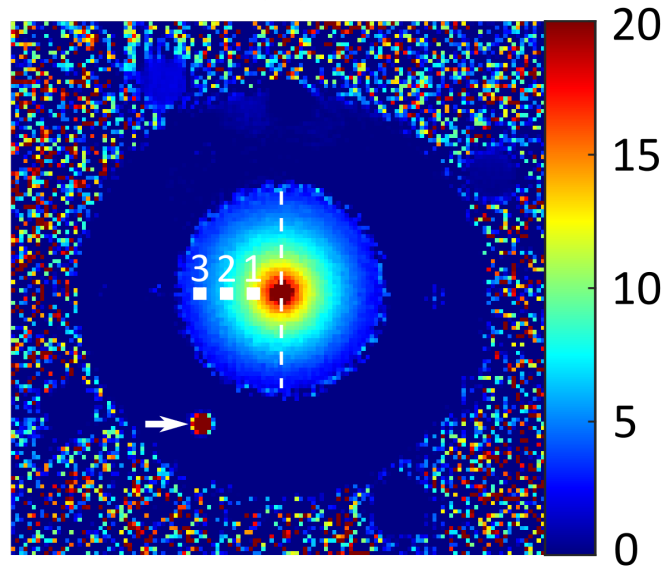

(d)

$\Delta \mathrm{T}\left({ }^{\circ} \mathrm{C}\right)$

Figure 4.5.: (a) A magnitude image of the temperature phantom at the hight of the three tips of the temperature sensors (shown by the arrows). Four reference tubes are located around the main phantom. (b) and (c) The neighboring slice of (a) before and after 2 hours of heating, respectively. (d) The relative temperature map calculated by the PRF method shows concentric increases of the temperature around the central tube. The temperature profile along the dashed line is illustrated in Figure 4.6. The temperature changes of the three ROIs located next to the tips of the fiber optic sensors (indicated by the three squares) are compared with the true temperature changes (Table 4.2). The high temperature increase observed in the cold water container (arrow) is due to the construction-conditioned out flowing warm water. 


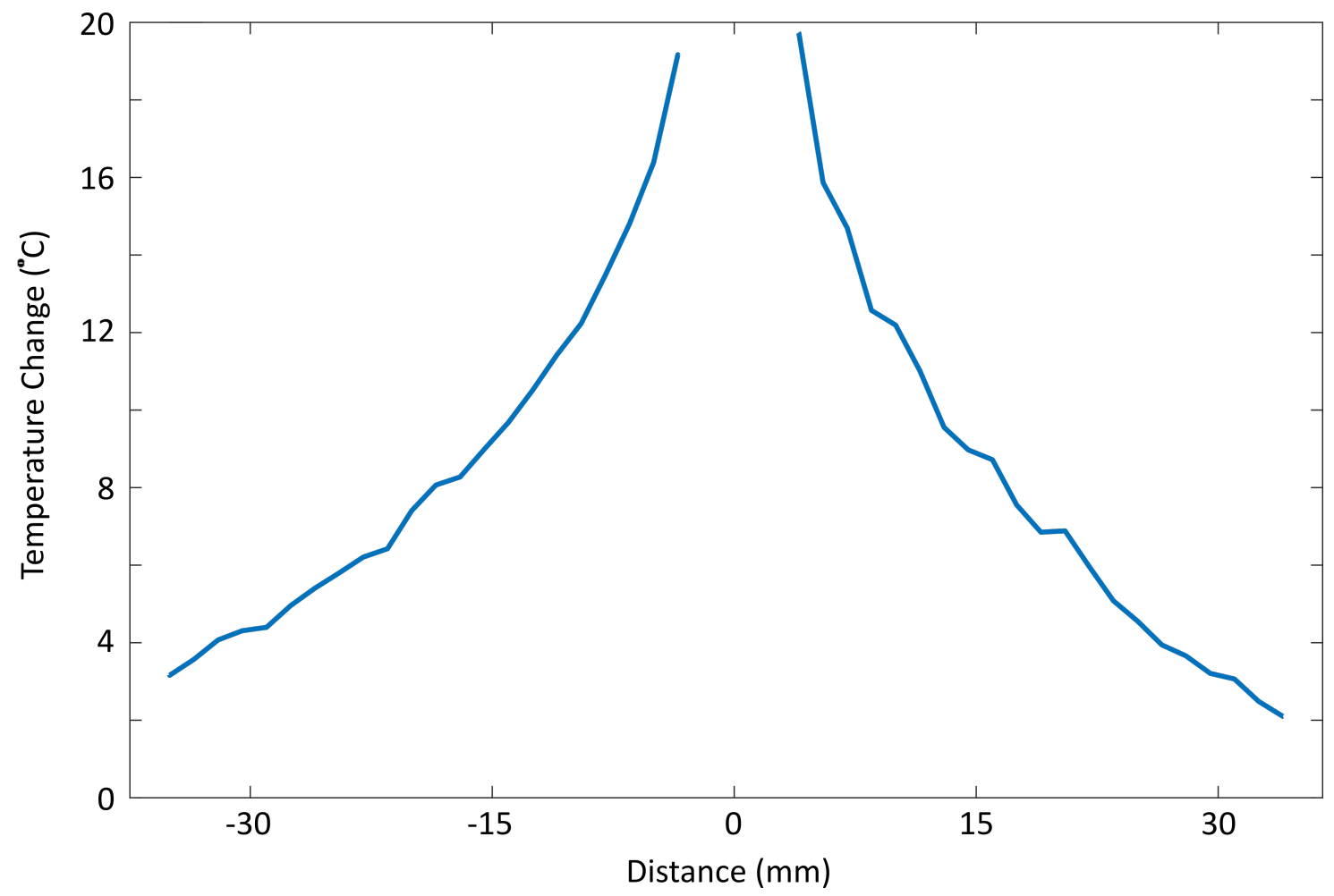

Figure 4.6.: The temperature profile of the dashed line in Figure 4.5 (d) obtained after two hours of heating. Values of the central tube are masked.

Table 4.2.: Comparison between the temperature changes of the three ROIs (see Figure 4.5 (d)) calculated by the PRF method and recorded by the fiber optic sensors.

\begin{tabular}{cccc}
\hline$\Delta \mathrm{T}\left({ }^{\circ} \mathrm{C}\right)$ & ROI 1 & ROI 2 & ROI 3 \\
\hline PRF thermometry & $11.6 \pm 0.9$ & $7.1 \pm 0.5$ & $3.9 \pm 0.3$ \\
\hline Fiber optic sensor & 12.2 & 7.1 & 3.8 \\
\hline
\end{tabular}



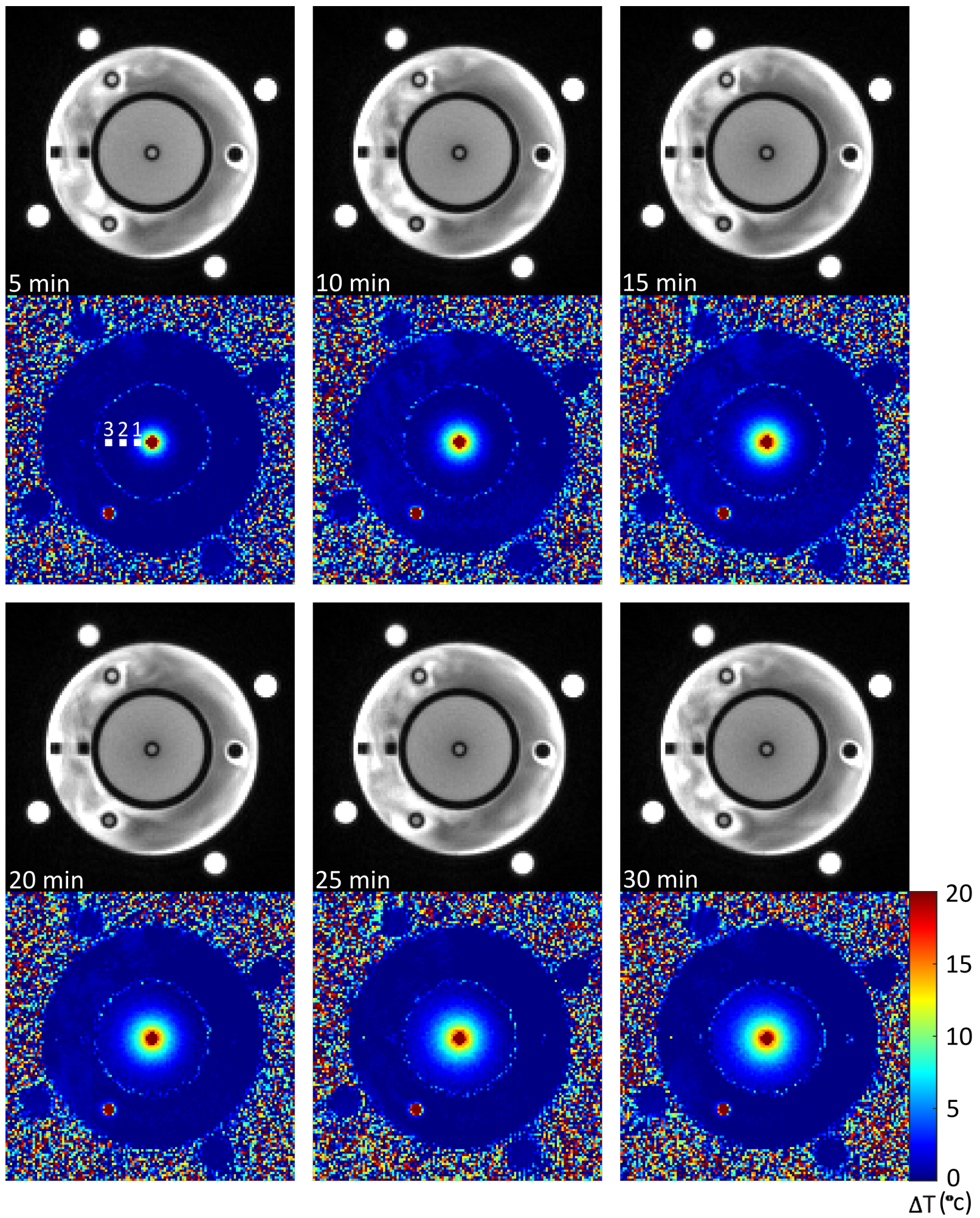

Figure 4.7.: Magnitude images and relative temperature difference maps selected examples from the movie during 30 minutes of heating. The temperature evolution of the three ROIs $(3 \times 3$ pixels, indicated by the three squares) are shown in Figure 4.8 . 


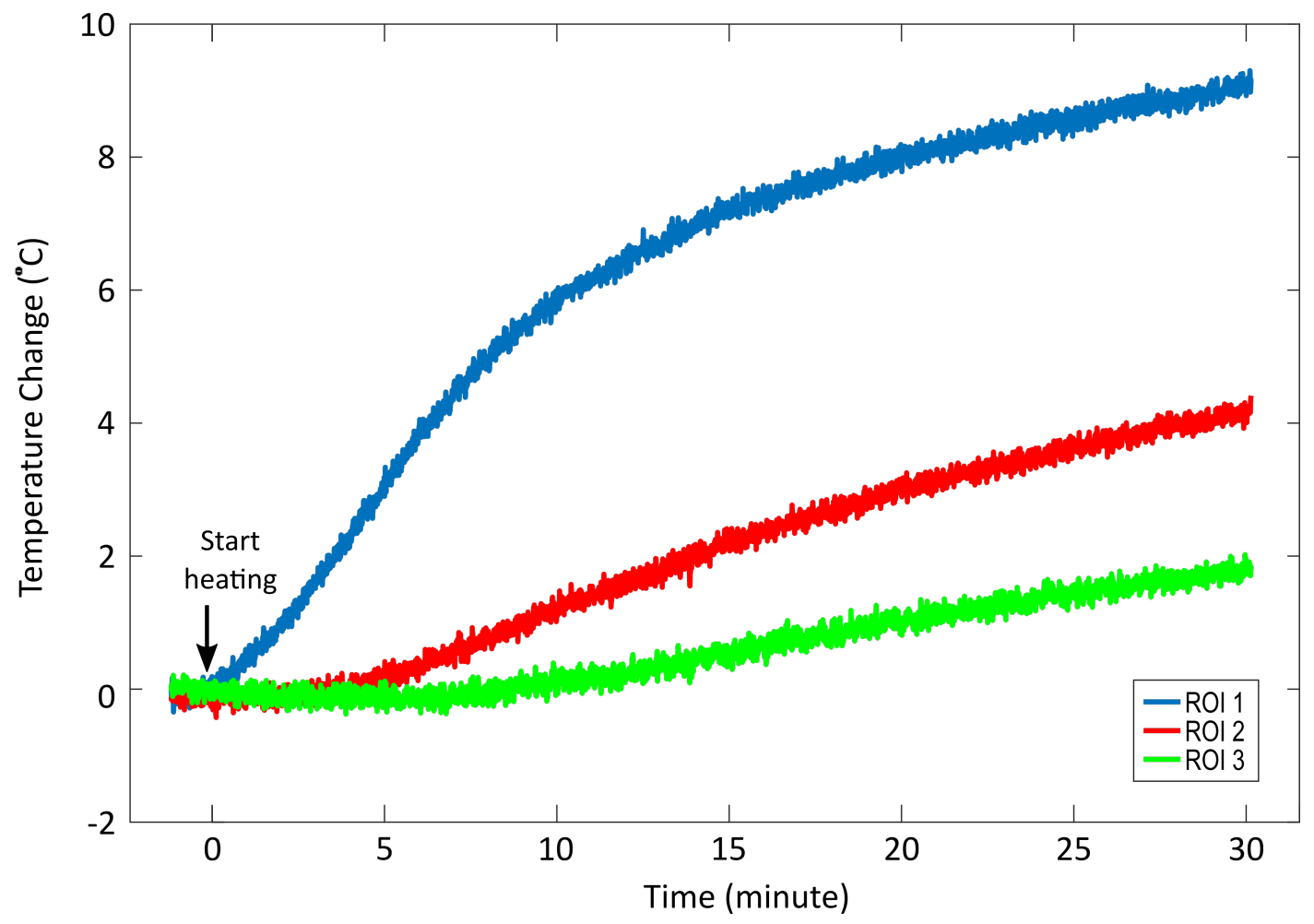

Figure 4.8.: Temperature change of the three ROIs (depicted in Figure 4.7) obtained during 30 minutes of heating.

\section{3. $\mathrm{T}_{1}$ Thermometry}

\subsubsection{Methods and Materials}

\section{Inversion Recovery Sequence}

Single shot inversion recovery (IR) $T_{1}$ mapping method is employed for quantitative $T_{1}$ thermometry. Such a method combines a $180^{\circ}$ pulse and the 'Look-Locker' technique $[92,93]$ for data acquisition. To be more specific, an IR module is applied prior to the data acquisition, which comprises a non-selective adiabatic RF pulse [94] that flips the magnetization with $180^{\circ}$ and a spoiling gradient that dephases the residue magnetization in the transversal plane (as shown in Figure 4.9). During the longitudinal relaxation, a highly undersampled radial FLASH sequence with a low flip angle is applied for signal acquisition to record the recovery process until the spins reach steady state. Moreover, in order to remove artifacts caused by the accumulated magnetization in the transverse plane, random RF is performed for signal excitation [95]. Note that a several seconds break has to be introduced between the neighboring IR acquisitions. The break should be longer than 5 times as the measured $T_{1}$ so that the magnetization can be fully relax. In the present work, a break time of $7 \mathrm{~s}$ is applied which leads to an updating time of $10 \mathrm{~s}$ for the $T_{1}$ method. More MR parameters are summarized in Table 4.3. 


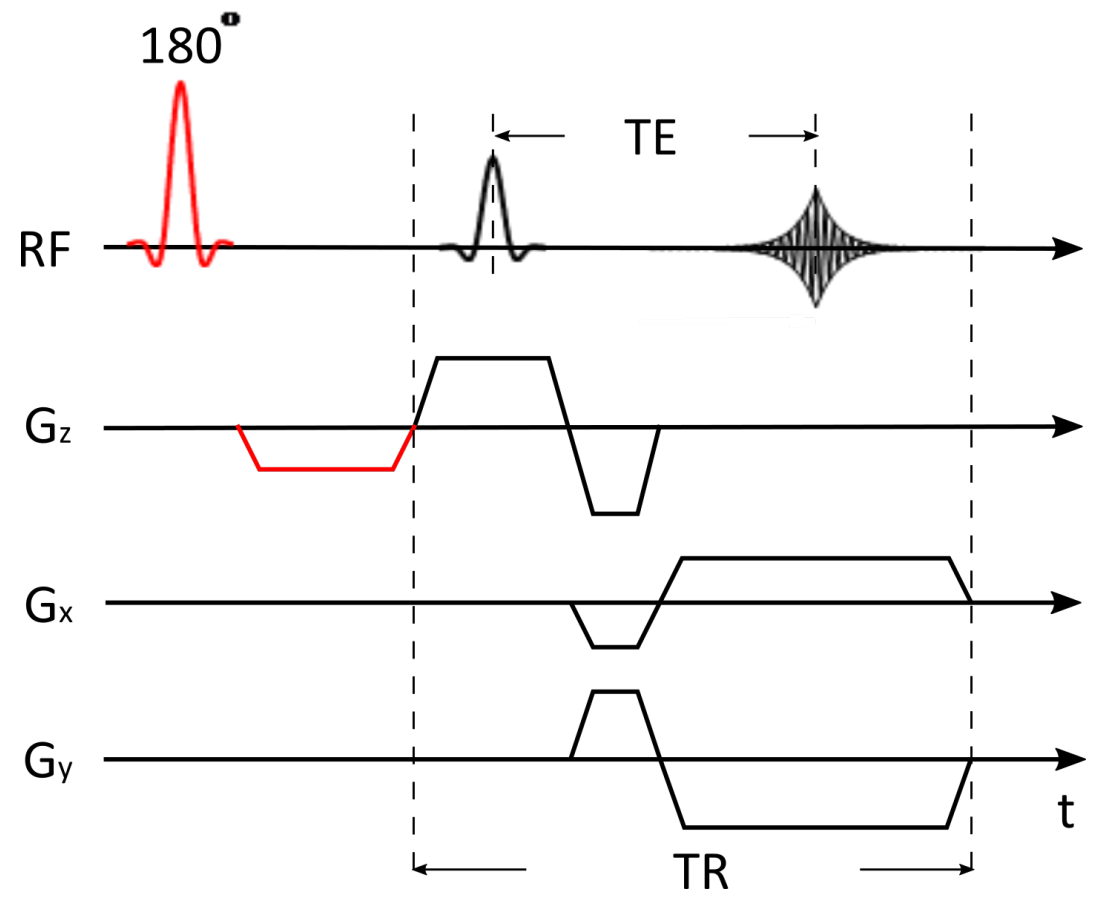

Figure 4.9.: The used single shot inversion recovery (IR) sequence consists of a IR module with a $180^{\circ} \mathrm{RF}$ pulse combined with a spoiling gradient (in red) and a radial FLASH sequence.

Table 4.3.: MR parameters of the single shot IR radial FLASH sequence for $T_{1}$ thermometry on the phantom.

\begin{tabular}{cc|cc}
\hline TE/TR & $1.25 / 2.1 \mathrm{~ms}$ & In-plane resolution & $1.5 \times 1.5 \mathrm{~mm}^{2}$ \\
\hline Flip angle & $4^{\circ}$ & Number of spokes & 19 per frame \\
\hline Slice thickness & $6 \mathrm{~mm}$ & Data acquisition time & $3 \mathrm{~s}$ \\
\hline FOV & $192 \times 192 \mathrm{~mm}^{2}$ & Used temporal resolution & $10 \mathrm{~s}$ \\
\hline
\end{tabular}

\section{Image Reconstruction}

NLINV Reconstruction for Inversion Recovery: Different from the signal acquired from steady state free precession (SSFP) measurements, data acquired by IR sequence covers the longitudinal relaxation which leads to a magnitude changes among the proton density images. As a consequence, for image reconstruction, NLINV takes the reverse chronological order to jointly estimate image content and coil profiles [42, 96]. To be 
more explicit, NLINV reconstruction is performed for the last ten frames of the acquired data, because the signal shows only slightly changes from one to the other and provide high quality coil sensitivities. According to Equation (2.33), once the coil profiles are fixed, the reconstruction can be considered as a linear inverse problem, because only the image content is the unknown parameter in the equation. Subsequently, the linear inverse problem is solved by the regularized iterative conjugate gradient $(\mathrm{CG})$ method. In the present work, 7 Newton steps and a damping factor of 0.7 are applied for computing coil sensitivities and 7 CG iterations and a damping factor of 0.7 are used for the following CG reconstruction.

Physical Model for Inversion Recovery: Under the disturbance of low flip angle $(\alpha)$ RF pulses, the relationship between the current $\left(M_{i}\right)$ and the next longitudinal magnetization $\left(M_{i+1}\right)$ can be expressed as:

$$
M_{i}=M_{s s}-\left(M_{0}+M_{s s}\right)\left[\cos (\alpha) \cdot e^{-\mathrm{TR} / T_{1}}\right]^{i}
$$

where $M_{s s}$ and $M_{0}$ are the steady state and the initial magnetization, respectively, TR is the sequence repetition time. Due to the fact that the applied TE is as short as $1.25 \mathrm{~ms}$ for data acquisition, the influence of the magnetization decay in the transversal plane can be neglected. Moreover, the effective relaxation time $\left(T_{1}^{*}\right)$ is given as:

$$
\frac{1}{T_{1}^{*}}=\frac{1}{T_{1}}-\frac{\ln (\cos (\alpha))}{\mathrm{TR}}
$$

As a consequence, Equation (4.5) can be rewritten as an exponential formula:

$$
M(t)=M_{s s}-\left(M_{s s}-M_{0}\right) \cdot e^{-t / T_{1}^{*}}
$$

with $t=i$. TR. Due to the fact that $\mathrm{TR} \ll T_{1}^{*}<T_{1}$, the true $T_{1}$ value can be derived from the following expression:

$$
T_{1}=T_{1}^{*} \cdot\left[\frac{M_{s s}-M_{0}}{M_{s s}}-1\right]
$$

Once the complex images of the IR process are calculated by the modified NLINV algorithm, an extended nonlocal mean algorithm [97] is applied on the images to further improve the image SNR. Based on Equation (4.7), a pixel-wise fitting is conducted on the denoised image series to determine $M_{s s}, M_{0}$, and $T_{1}^{*}$. Finally, the desired $T_{1}$ is yielded by the determined parameters and Equation (4.8). 


\section{Experimental Procedures}

The location of the phantom and temperature setting of the cycled water followed the same description in Chapter 4.2 and sequence protocols in Table 4.3 were used for data acquisition.

Temperature Dependency of $\mathbf{T}_{\mathbf{1}}$ : In order to determine the temperature dependency of $T_{1}$ of the agarose phantom, one $T_{1}$ map was acquired before switching on the pumps. After the two pumps working for two hours, another $T_{1}$ map was measured. The temperature sensitivity of $T_{1}$ was calculated based on the $T_{1}$ value and the temperature changes recorded from the fiber optic sensors.

Dynamic $\mathbf{T}_{1}$ Thermometry: For the purpose of monitoring $T_{1}$ development in realtime, the two pumps and the single shot IR sequence train were started simultaneously for half an hour. A $T_{1}$ movie was obtained based on Equation (4.7) and (4.8).

\subsubsection{Results}

$T_{1}$ maps used for the temperature sensitivity calculation are illustrated in Figure 4.10. $T_{1}$ values in the agarose container obtained before heating are about $1000 \mathrm{~ms}$ (Figure 4.10 (a)). Similar to the results obtained by PRF method, the $T_{1}$ values acquired after two hours heating shows a symmetrical distribution to the central tube (Figure 4.10 (b)). In addition, Figure 4.11 shows the $T_{1}$ profiles along the reference line acquired before and after heating. Again, $T_{1}$ values acquired before heating are about $1000 \mathrm{~ms}$. For the profile acquired after heating, the $T_{1}$ near the central tube is increased to about $1400 \mathrm{~ms}$ and a $T_{1}$ value of about $1100 \mathrm{~ms}$ can be observed at the outer edge. Moreover, temperature sensitivity of $T_{1}$ is shown in Table 4.4, which is calculated from the recorded temperature changes by the fiber optic sensors and the $T_{1}$ values of the corresponding ROIs (indicated in Figure 4.10 (a), chosen in the same way as described in Chapter 4.2). All the ROIs show an approximate $2 \% /{ }^{\circ} \mathrm{C}$ sensitivity, which matches the results from other studies [75]. The $T_{1}$ uncertainties indicated by SDs are below 2.5\%. The analysis of SDs in the $T_{1}$ difference maps will be performed in the ex vivo studies.

As for the dynamic $T_{1}$ mapping experiment, half an hour data acquisition yields 180 frames in total. The $T_{1}$ maps for every five minutes are displayed in Figure 4.12. The development of the $T_{1}$ gradient can be observed. In general, the $T_{1}$ shows a similar development as the results acquired with PRF method. With the increase of the measurement time, the $T_{1}$ near the central tube increases higher than that near the edge of the agarose container. Additionally, $T_{1}$ development of the three ROIs during heating are shown in Figure 4.13. Similar to the results obtained with the fiber optic sensors, due to the 


\section{Real-time MR Thermometry - Phantom Studies}

different relative distances to the central tube, a quick $T_{1}$ increase is observed with ROI 1 and the $T_{1}$ is increased to $1150 \mathrm{~ms}$ at ten minutes and $1230 \mathrm{~ms}$ at 30 minutes. Delayed increases are recorded with ROI 2 and 3, and the $T_{1}$ is increased to $1100 \mathrm{~ms}$ and $1060 \mathrm{~ms}$ at 30 minutes, respectively.

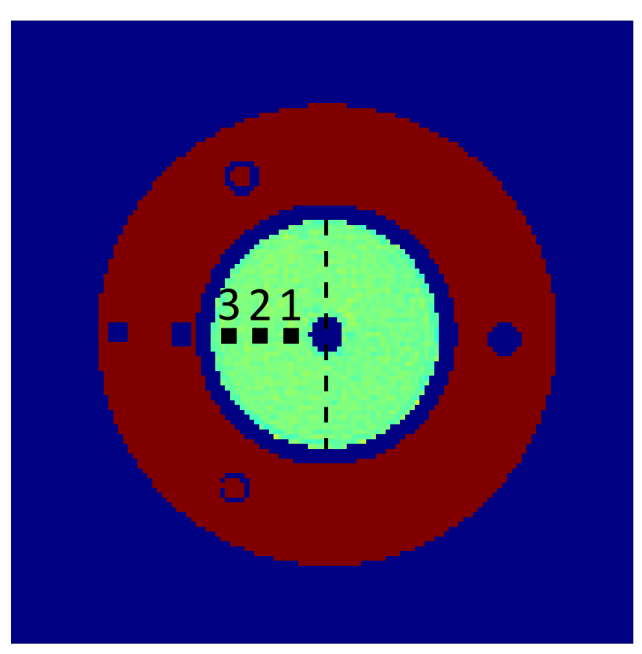

(a)

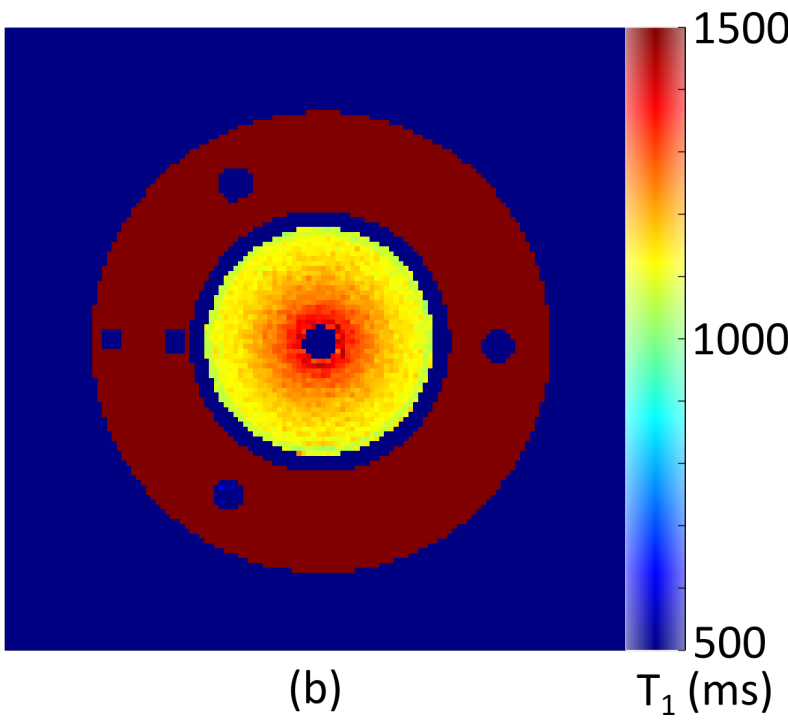

(b) (ms)

Figure 4.10.: $T_{1}$ maps acquired with the single shot IR radial FLASH sequence. (a) $T_{1}$ map before heating. (b) $T_{1}$ map after 120 minutes of heating. $T_{1}$ profiles along the dashed line before and after heating are shown in Figure 4.11. The $T_{1}$ values of the three ROIs (squares) are summarized Table 4.4.

Table 4.4.: Evaluation of the temperature sensitivity of $T_{1}$. The three ROIs are depicted in Figure 4.10.

\begin{tabular}{cccc}
\hline & ROI 1 & ROI 2 & ROI 3 \\
\hline $\begin{array}{c}T_{1} \\
\text { (before heating) }\end{array}$ & $1005 \pm 17 \mathrm{~ms}$ & $995 \pm 17 \mathrm{~ms}$ & $1003 \pm 16 \mathrm{~ms}$ \\
\hline $\begin{array}{c}T_{1} \\
\text { (after 120 min of heating) }\end{array}$ & $1303 \pm 31 \mathrm{~ms}$ & $1197 \pm 20 \mathrm{~ms}$ & $1123 \pm 18 \mathrm{~ms}$ \\
\hline $\begin{array}{c}\text { Temperature change } \\
\text { (from fiber optic sensor) }\end{array}$ & $14.8{ }^{\circ} \mathrm{C}$ & $9.7{ }^{\circ} \mathrm{C}$ & $6.4{ }^{\circ} \mathrm{C}$ \\
\hline $\begin{array}{c}T_{1} \text { change } \\
\text { per }{ }^{\circ} \mathrm{C}\end{array}$ & $2.0 \% /{ }^{\circ} \mathrm{C}$ & $2.1 \% /{ }^{\circ} \mathrm{C}$ & $1.9 \% /{ }^{\circ} \mathrm{C}$ \\
\hline
\end{tabular}




\subsection{Summary}

Three major tasks were accomplished for real-time MRI temperature mapping in this chapter. First of all, a suitable phantom for MR thermometry has been developed. The phantom is $\mathrm{MR}$ compatible and has a homogeneous $T_{1}$ value of around $1000 \mathrm{~ms}$ (at room temperature). More importantly, a temperature gradient of at least $8{ }^{\circ} \mathrm{C}$ can be generated from the fiber optic sensor 1 to 3 . Secondly, PRF thermometry was set up for the phantom study. Radial FLASH sequence was optimized with respect to the determination of an optimal TE as well as the application of an additional flow compensation gradient. For image reconstruction, images are reconstructed from the undersampled MR raw data by the NLINV algorithm with proper Newton steps and the damping factor. A multi-channel phase-contrast algorithm has been implemented to yield corresponding temperature difference maps. As illustrated in the results, with PRF approach, a temperature updating time of $200 \mathrm{~ms}$ is achieved and the calculated temperature changes from different ROIs match the data recorded from fiber optic sensors very well. Furthermore, $T_{1}$ mapping method is also developed for dynamic temperature mapping. Single shot IR sequence is employed for data acquisition. A modified NLINV algorithm is adopted for

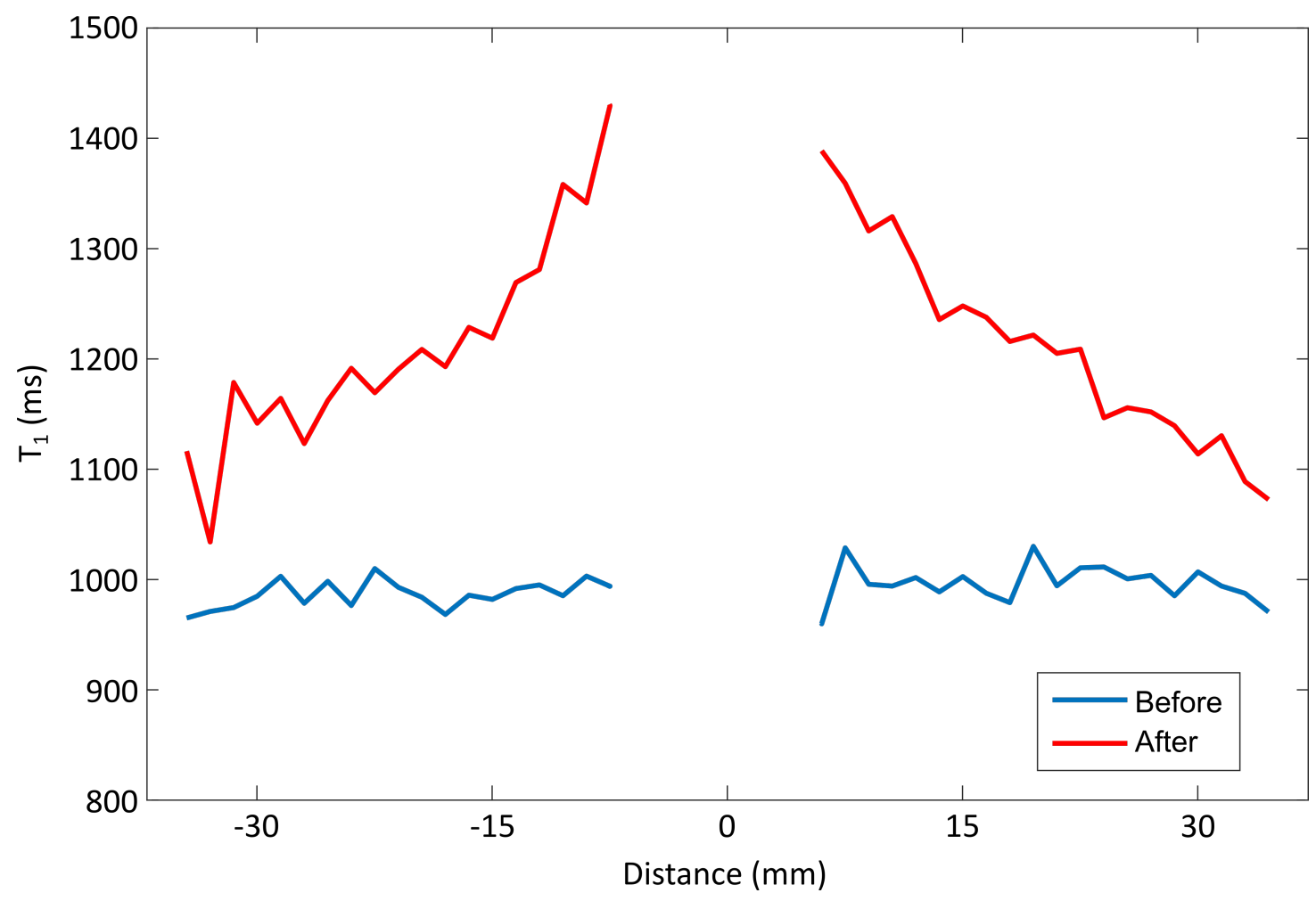

Figure 4.11.: The $T_{1}$ profiles of the dashed line in Figure 4.10 (a) obtained before and after two hours of heating. Values in the central tube are masked. 


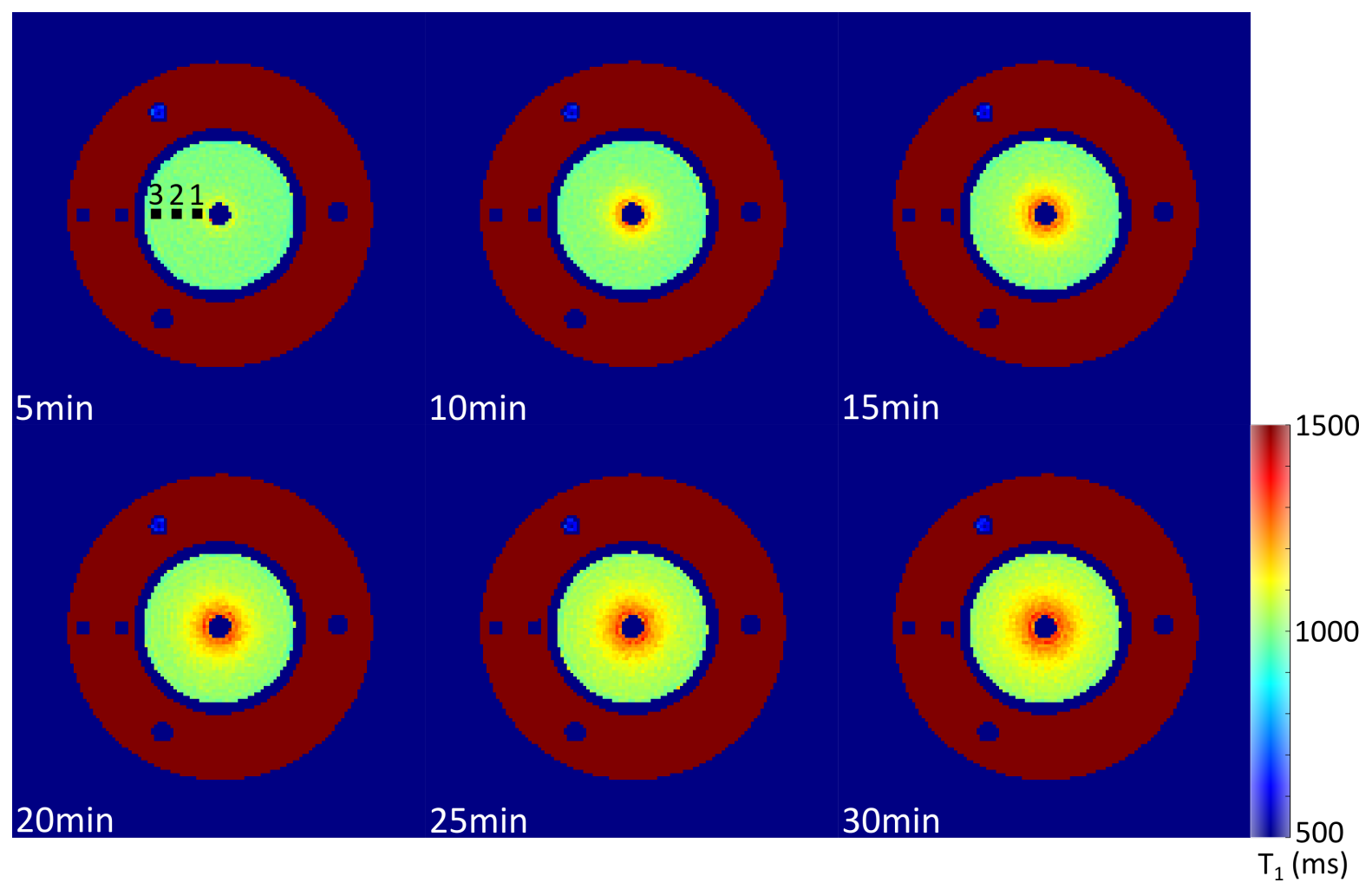

Figure 4.12.: $T_{1}$ maps selected examples from the movie during 30 minutes of heating. The $T_{1}$ changes of the three ROIs $(3 \times 3$ pixels, indicated by the three squares $)$ are shown in Figure 4.13.

image reconstruction. As for the results, with $T_{1}$ method, the temporal resolution of $T_{1}$ map is $10 \mathrm{~s}$ in the phantom study and the pixel wise fitted $T_{1}$ value shows a temperature dependency of $2 \% /{ }^{\circ} \mathrm{C}$, which agrees with the results from other studies [75]. Therefore, it can be concluded that both of the two methods show promising results and abilities for dynamic temperature mapping when combined with real-time MRI. A comprehensive comparison between the two methods will be investigated in the next chapter on ex vivo tissues. 


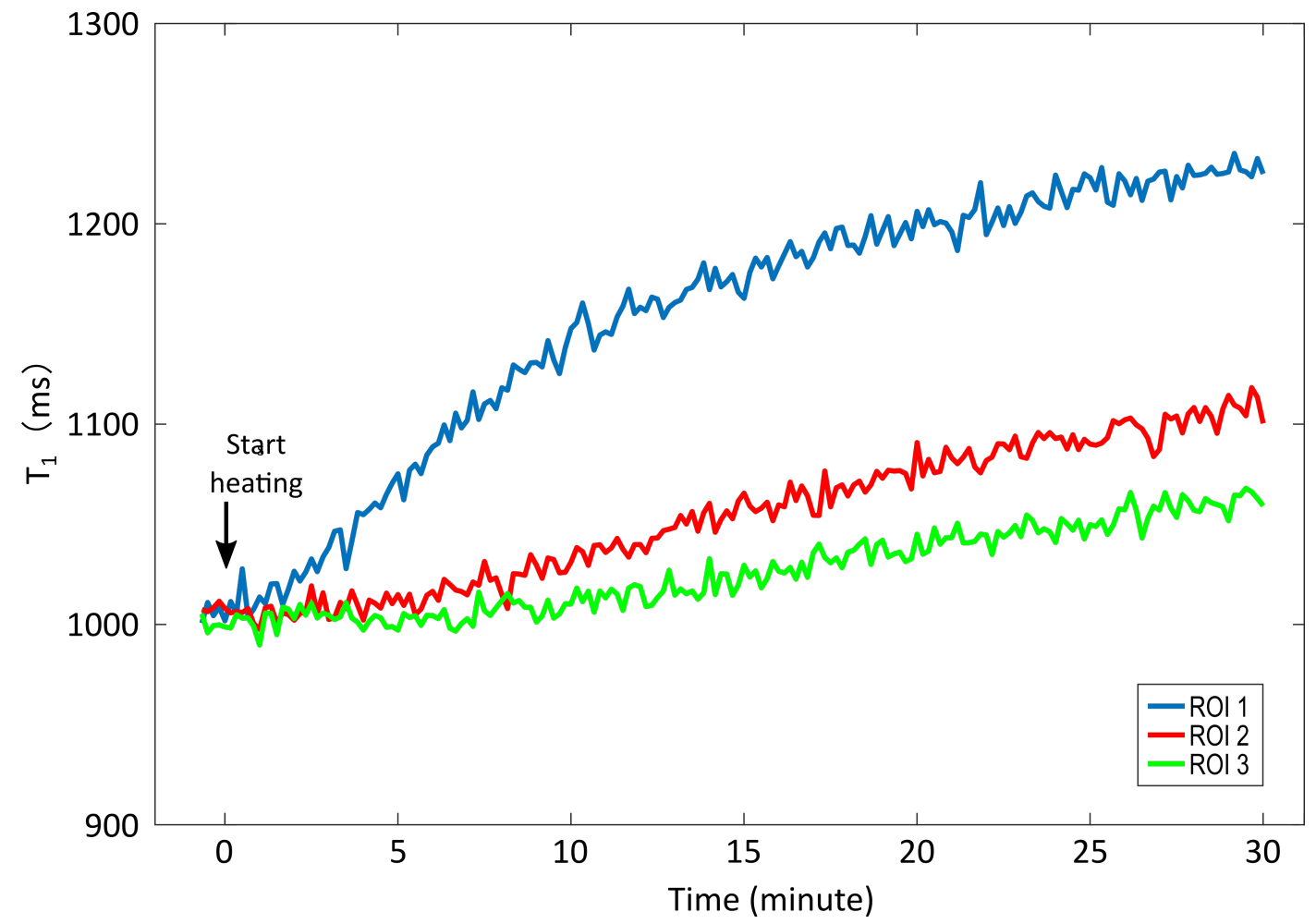

Figure 4.13.: $T_{1}$ changes of three ROIs (depicted in Figure 4.12) obtained during 30 minutes of heating. 



\section{Real-time MR Thermometry - Ex Vivo Tissue Studies with Global Heating}

In the last chapter, both $\mathrm{PRF}$ and $T_{1}$ methods show the ability of dynamic temperature mapping in phantom studies when combined with real-time MRI. Therefore, a further comprehensive investigation especially with respect to ex vivo studies has to be launched in order to compare these two methods. In the first part of this chapter, the kidney is chosen as the experimental subject for the comparison, because it receives a major interest in tissue thermal therapy [98, 99]. The complex physiological structure, which consists of cortex, medulla and a complex vascular system (Figure 5.1), offers more information during MR thermometry. In the second part of this chapter, the temperature accuracy is assessed again on kidney as well as liver of swine. Subsequently, temperature mapping on the tissue containing layers of lipid and muscle is also demonstrated.

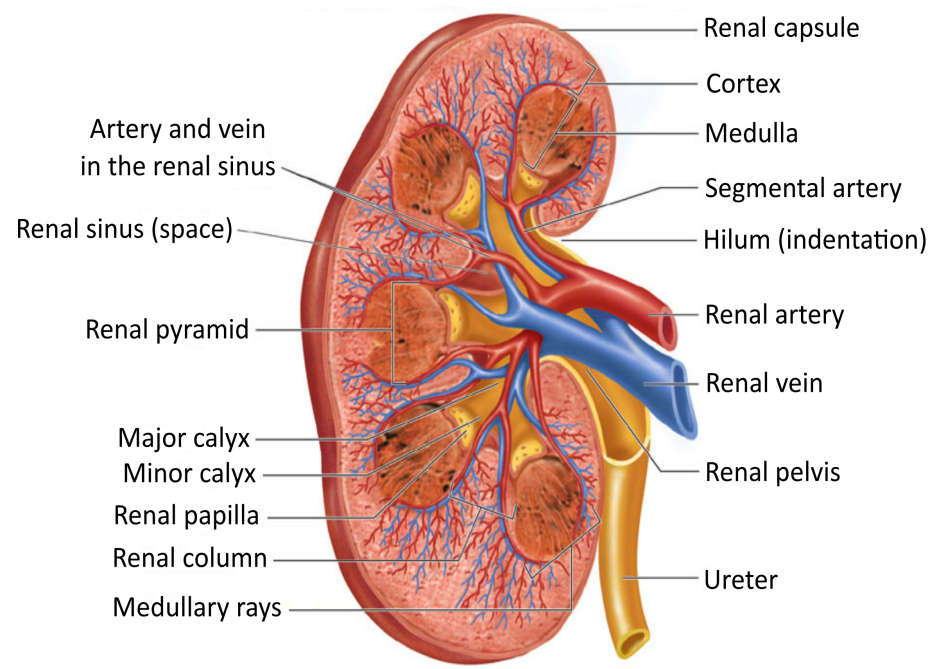

(a)

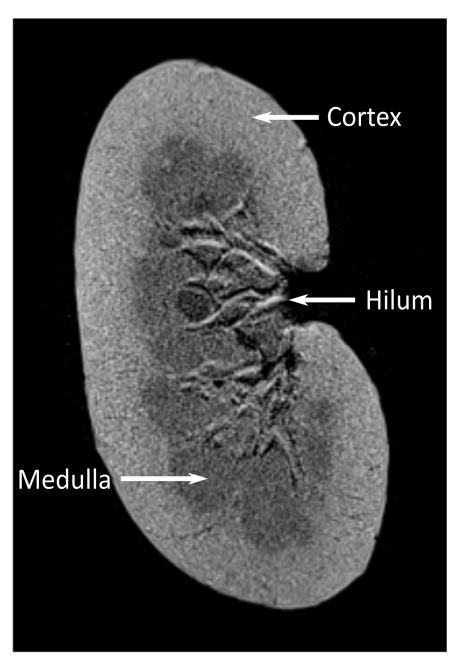

(b)

Figure 5.1.: (a) Anatomy of the human kidney. ${ }^{1}$ (b) $T_{1}$ weighted MR image of a swine kidney with an isotropic spatial resolution of $0.5 \times 0.5 \times 0.5 \mathrm{~mm}^{3}$.

\footnotetext{
${ }^{1}$ www.oustormcrowd.com
} 


\subsection{Comparison between PRF Shift and $T_{1}$ Methods}

\subsubsection{Experimental Setup}

\section{Organ Model and Heating Method}

Fresh porcine kidneys purchased from local supermarkets were used for ex vivo experiments. A water tube (inner diameter: $4 \mathrm{~mm}$, wall thickness: $1 \mathrm{~mm}$ ), which is connected to a pump that provides warm water, was inserted into the kidney along its long axis (Figure 5.2). After covered with plastic wrap, the kidney was positioned in the scanner at least one hour before the experiments to reach thermal steady state. During the experiment, the heating device was switched on for half an hour. The temperature of the cycled water was $70^{\circ} \mathrm{C}$.

\section{MRI Imaging}

MR sequences, which consists of PRF and $T_{1}$ sampling scheme in both the coronal and the transverse planes (image orientations seen in Figure 5.2), were employed for data acquisition. Related protocol details are summarized in Table 5.1. MR data acquired before heating was used as the baseline. Once the pump was turned on, the sequences were started in an interleave scheme every $5 \mathrm{~min}$ up to one hour, so that temperature changes in the kidney can be monitored by both methods during and after the heating procedure. Temperature maps of PRF and $T_{1}$ thermometry were reconstructed as described in Chapter 4. In order to compare the temperature difference maps acquired with the PRF method, $T_{1}$ difference maps were calculated. The $T_{1}$ map acquired before heating was used as the reference. Due to the duration of each acquisition is less than one minute, one can ignore the minor temperature change within each measurement.

\subsubsection{Results}

As shown in Figure 5.2 (a), the water tube is visible in the upper half of the kidney. Coronal and transversal temperature difference maps obtained by the PRF method are depicted in Figure 5.3 and 5.4, respectively. For both imaging orientations, the temperature differences of the kidney show a symmetrical distribution around the water tube. Moreover, during the heating period, in the coronal maps no differences can be observed between cortex and medulla. Outer parts of the kidney do not show temperature changes. Figure 5.5 (a) shows the temperature profile along a reference line (indicated in Figure 5.4 ) acquired after $30 \mathrm{~min}$ of heating. The temperature of the pixels close to the tube is increased by around $15{ }^{\circ} \mathrm{C}$, while no temperature changes can be observed in the outer parts of the kidney. During the period after heating, thermal energy is still transfered from the high to the low temperature area to reach thermal equilibrium. Therefore, in 
Table 5.1.: MR parameters to compare PRF and $T_{1}$ thermometry on ex vivo studies.

\begin{tabular}{cc|cc}
\hline \multicolumn{4}{c}{ PRF thermometry } \\
\hline Sequence & Radial FLASH & In-plane resolution & $1.5 \times 1.5 \mathrm{~mm}^{2}$ \\
\hline TE/TR & $10 / 11.8 \mathrm{~ms}$ & Slice thickness & $6 \mathrm{~mm}$ \\
\hline Flip angle & $10^{\circ}$ & Number of spokes & 17 per frame \\
\hline FOV & $192 \times 192 \mathrm{~mm}^{2}$ & Temporal resolution & $200 \mathrm{~ms}$ \\
\hline \hline \multicolumn{4}{c}{$T_{1}$ thermometry } \\
\hline Sequence & IR radial FLASH & In-plane resolution & $1.5 \times 1.5 \mathrm{~mm}^{2}$ \\
\hline TE/TR & $1.25 / 2.1 \mathrm{~ms}$ & Slice thickness & $6 \mathrm{~mm}$ \\
\hline Flip angle & $4^{\circ}$ & Number of spokes & 19 per frame \\
\hline FOV & $192 \times 192 \mathrm{~mm}^{2}$ & Data acquisition time & $3 \mathrm{~s}$ \\
\hline
\end{tabular}

the last temperature maps which are acquired 30 min after turning off the pump, the calculated temperature changes are still above $0{ }^{\circ} \mathrm{C}$ (room temperature), and this finding is confirmed in Figure 5.5 (b). The temperature changes of the three ROIs (indicated in Figure 5.4) are shown in Figure 5.6. The temperature of ROI 1 ( $0.5 \mathrm{~cm}$ to the tube) is increased by $10{ }^{\circ} \mathrm{C}$ and $14{ }^{\circ} \mathrm{C}$ after 5 and 30 min heating, respectively and drops to a temperature difference of $6{ }^{\circ} \mathrm{C}$ after switching off the pump for $30 \mathrm{~min}$. The SDs of ROI 1 during heating are higher than the ones after heating, which is due to the fact that the chosen ROI is close to the water tube and there is a considerable temperature difference within the ROI. The temperature of ROI 2 (2 cm to the tube) and ROI $3(3.5 \mathrm{~cm}$ to the tube) is raised by $5{ }^{\circ} \mathrm{C}$ and $1{ }^{\circ} \mathrm{C}$ after $30 \mathrm{~min}$ heating, respectively. The temperature in ROI 3 still increases during the period after heating.

Coronal and transversal $T_{1}$ difference maps acquired by single shot IR sequence are shown in Figures 5.7 and 5.8, respectively. For both imaging orientations, $T_{1}$ difference increases with the heating time. However, in contrast to the PRF method, it shows an asymmetrical distribution around the water tube. During the heating period, the $T_{1}$ in the kidney medulla raises more than that in the kidney cortex. ROI A and ROI B (in Figure 5.8) are chosen from the kidney cortex and medulla at 30 min of heating, respectively, which have the same distance to the tube $(7.5 \mathrm{~mm}) . T_{1}$ of the two ROIs are increased by $21 \%$ and $33 \%$, respectively. Figure 5.9 (a) shows the $T_{1}$ difference profile along a reference line (shown in Figure 5.8) after 30 min of heating. The $T_{1}$ of the pixels close to the tube is increased by $600 \mathrm{~ms}$ and the $T_{1}$ is raised by around $100 \mathrm{~ms}$ in the outer parts of the kidney. During the period after heating, the $T_{1}$ difference shows an asymmetrical distribution around the water tube. As illustrated in Figure 5.9 (b), the remaining $T_{1}$ difference in the medulla is around $350 \mathrm{~ms}$ which is higher than that in the cortex (around $150 \mathrm{~ms}$ ) 


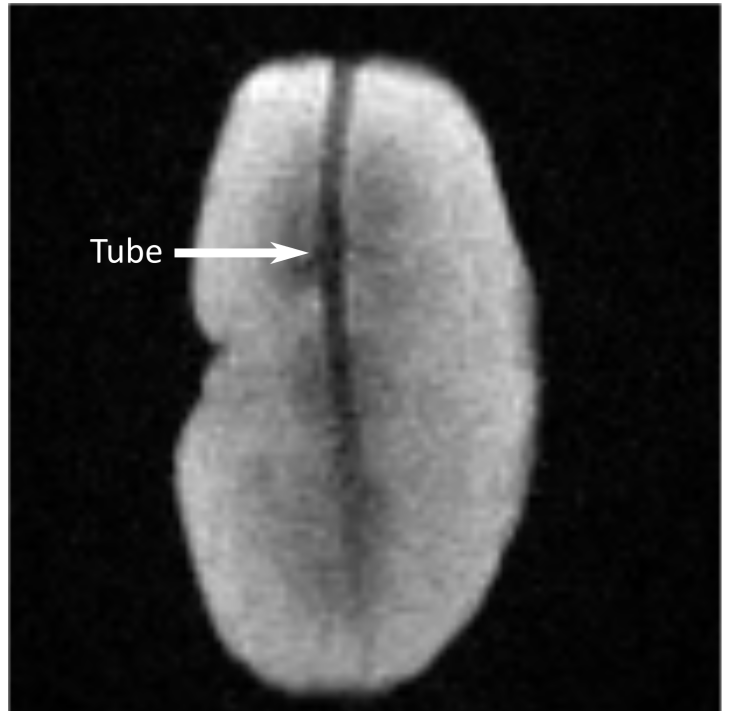

(a)

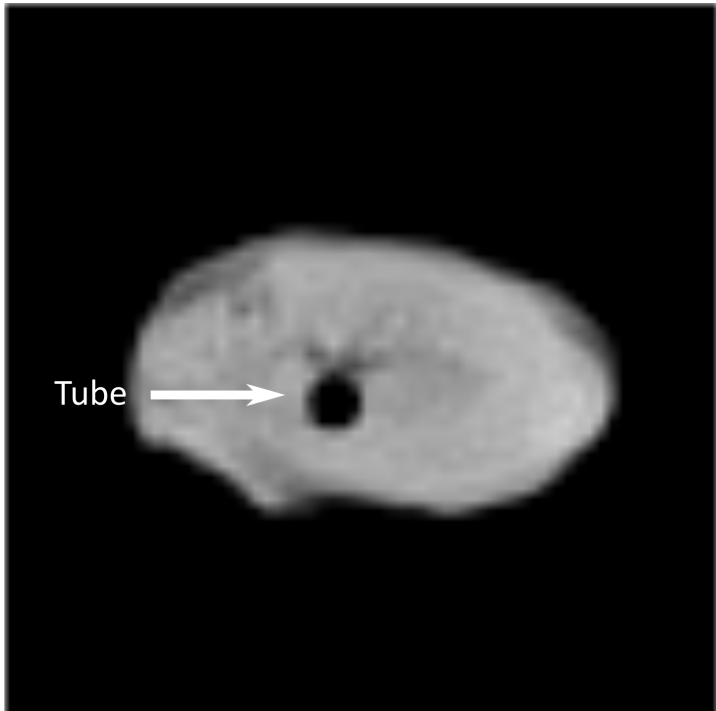

(b)

Figure 5.2.: (a) Coronal and (b) transversal $T_{1}$ weighted MR images of a kidney (spatial resolution of $\left.1.5 \times 1.5 \times 6 \mathrm{~mm}^{3}\right)$. These image orientations parallel and orthogonal to the tube are used for PRF and $T_{1}$ thermometry.

after 30 min of heating. In addition, the $T_{1}$ difference of the three ROIs (indicated in Figure 5.8) are shown in Figure 5.10. The mean values of the three ROIs show similar behavior as the PRF method, however, the corresponding SDs are very high. This results in a high temperature uncertainty of the $T_{1}$ method (above $30 \%$ ).

\subsubsection{Summary}

In general, both PRF and $T_{1}$ methods show sensitivities to temperature in the swine kidney when combined with real-time MRI. However, the PRF method is superior to the $T_{1}$ method for three reasons. First of all, the temperature sensitivity of PRF is a constant value in all the aqueous tissues and it reflects the real temperature distribution, whereas the temperature sensitivity of $T_{1}$ is tissue type dependent and needs to be evaluated for every tissue of interests before the heating procedure. In the temperature difference maps acquired by the PRF method, the temperature differences show a symmetric distribution around the water tube. Secondly, the temperature updating time for the PRF method is $200 \mathrm{~ms} /$ frame, while $T_{1}$ updating time is $10 \mathrm{~s}(3 \mathrm{~s}$ for data acquisition and $7 \mathrm{~s}$ for fully magnetization relaxation). The duration of minimally invasive tissue ablation or coagulation surgeries can be as short as several min and a temperature increase of more than $5{ }^{\circ} \mathrm{C}$ can be observed in $10 \mathrm{~s}$ [100]. Therefore, the temporal resolution of the $T_{1}$ method is not adequate to monitor rapid temperature changes. Thirdly, the temperature maps calculated with the PRF method have a higher SNR than that computed with the 


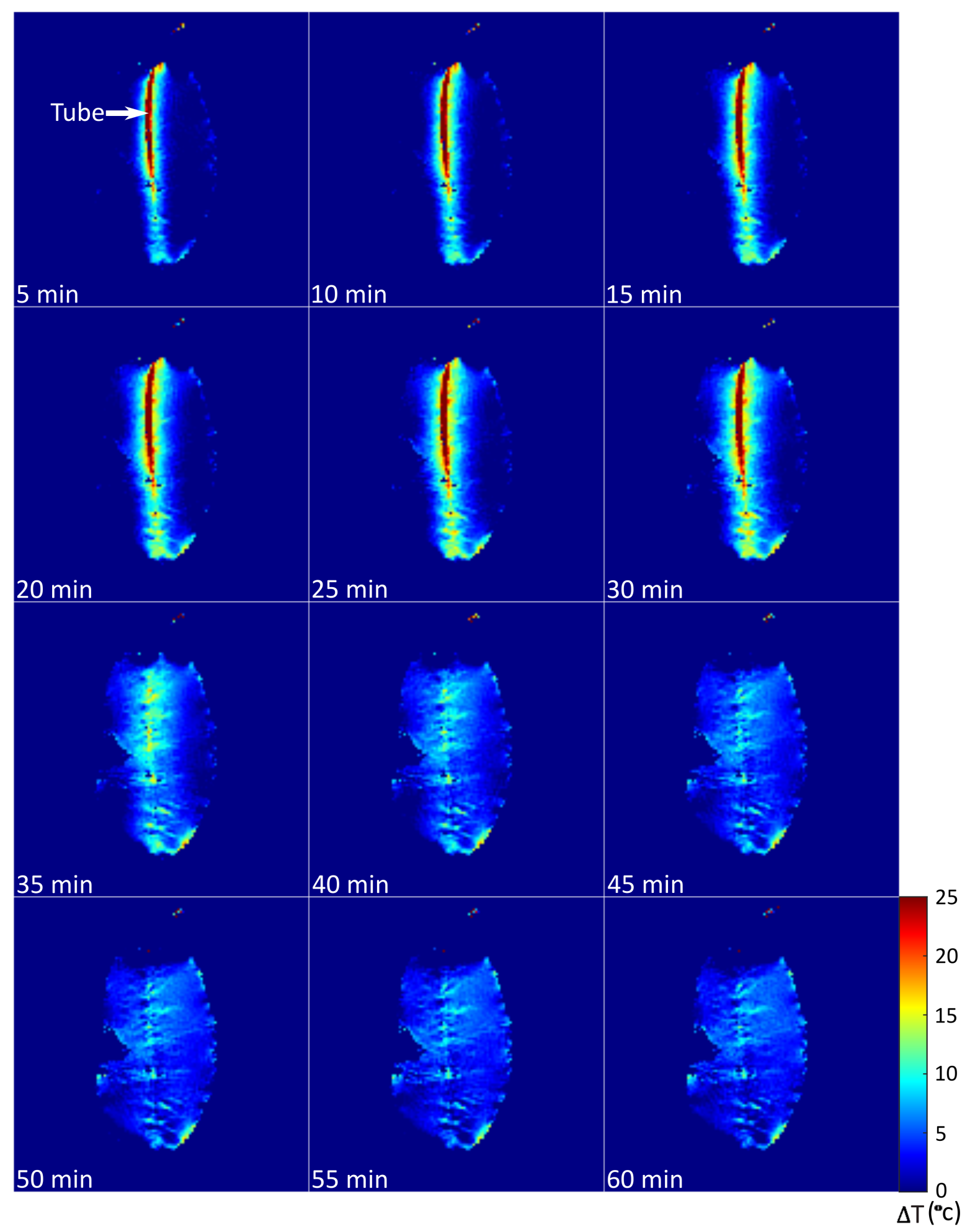

Figure 5.3.: Coronal temperature difference maps of the swine kidney obtained every 5 min during and after 30 min of heating. 


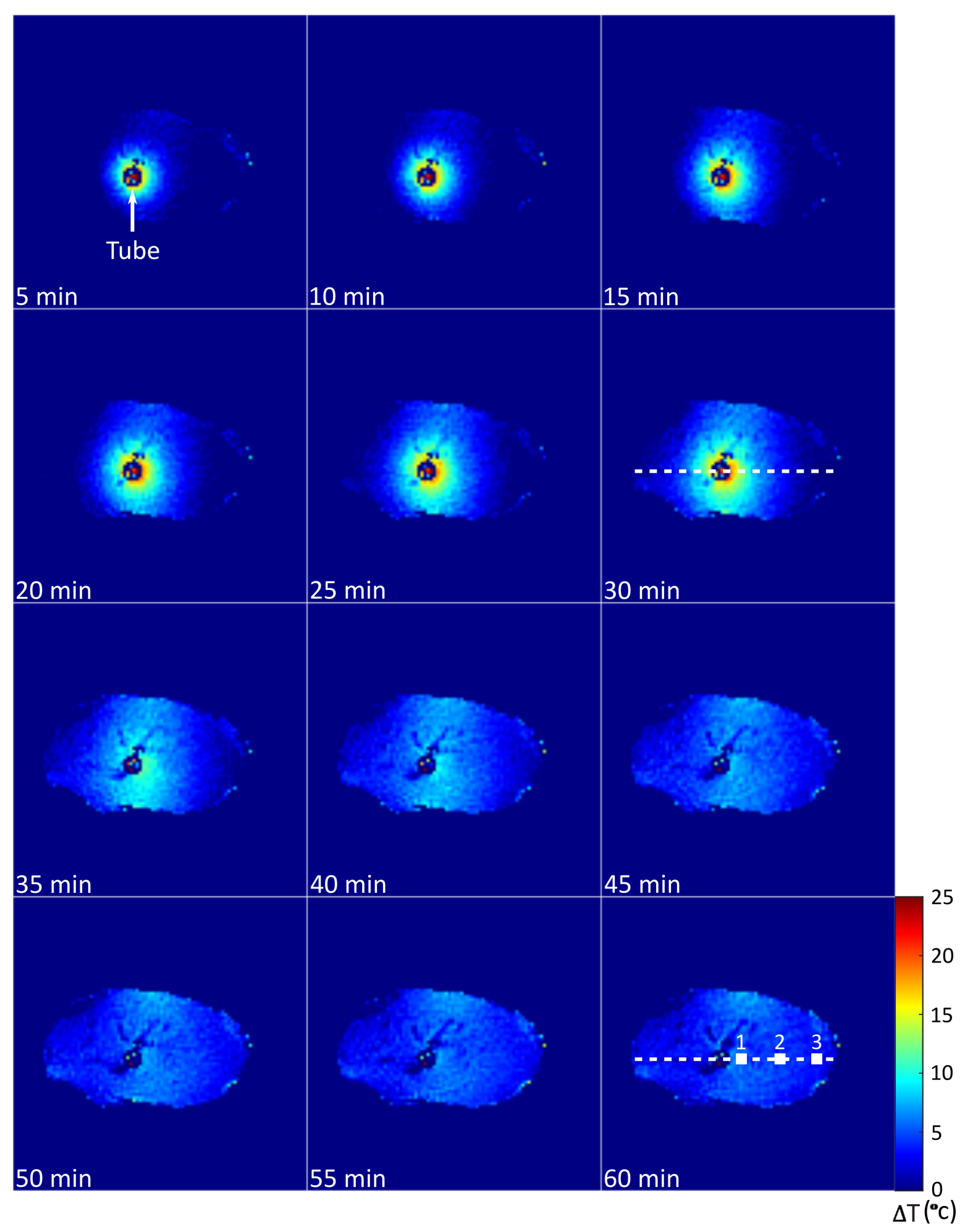

Figure 5.4.: Transversal temperature difference maps of the swine kidney obtained every 5 min during and after 30 min of heating. The temperature profile along the two dashed line is illustrated in Figure 5.5. The temperature changes of the three ROIs (indicated by the three squares) are shown in Figure 5.6. 


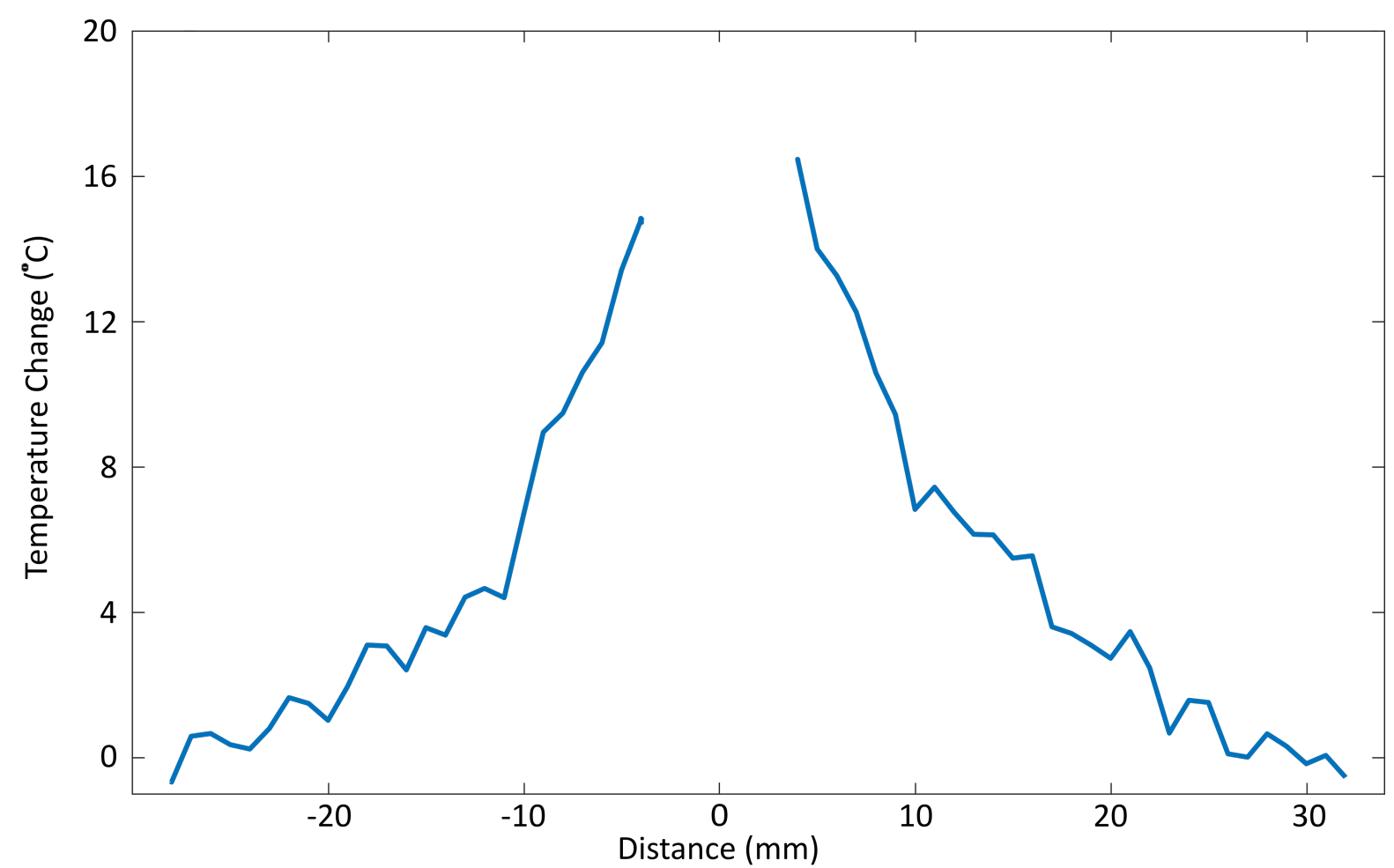

(a)

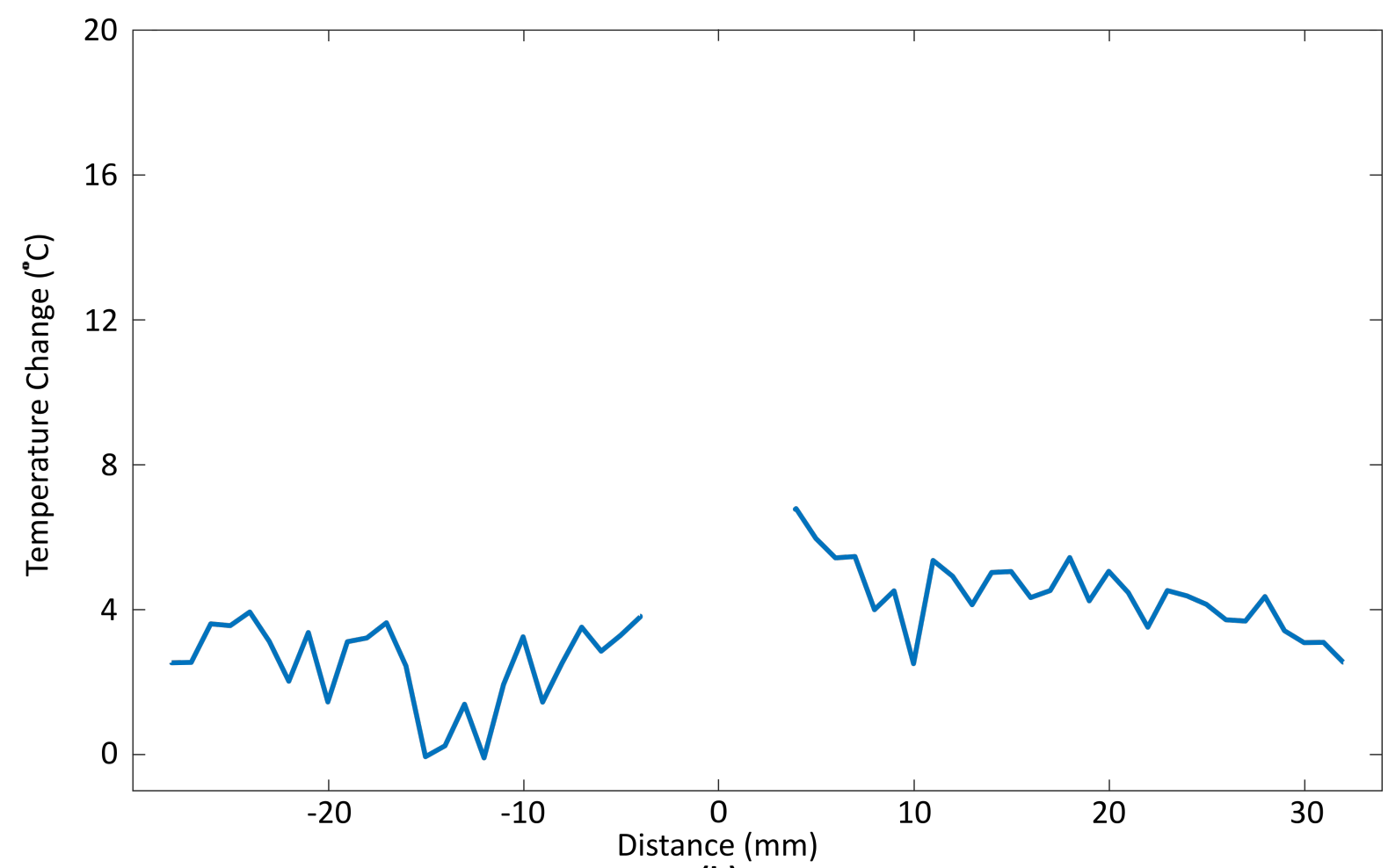

(b)

Figure 5.5.: (a) Temperature profile along the dashed line (indicated in Figure 5.4) obtained after 30 min of heating. (b) Temperature profile along the dashed line (indicated in Figure 5.4) obtained after 30 min after heating. Values of the central tube are masked. 


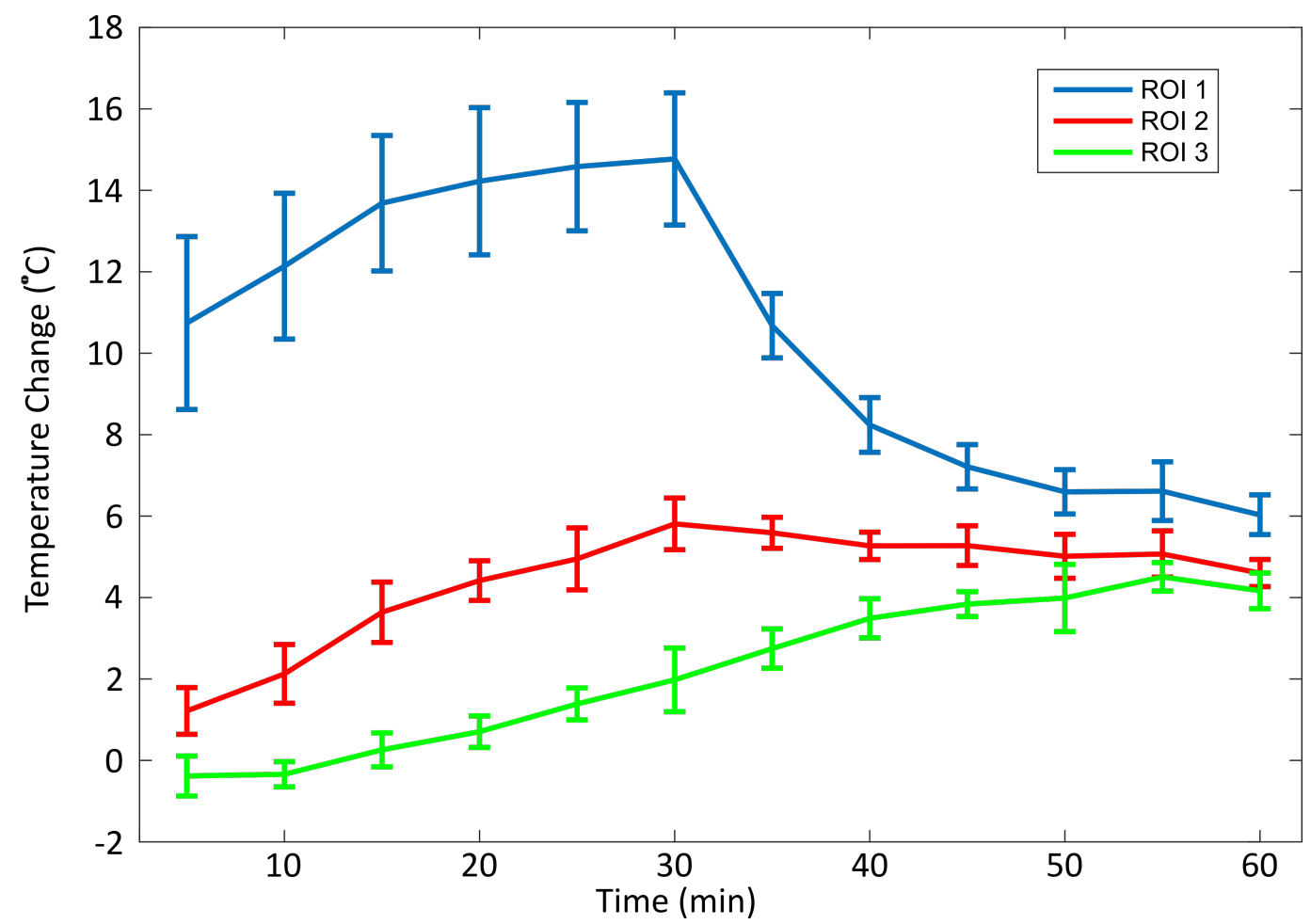

Figure 5.6.: Temperature changes (mean $\pm \mathrm{SD}$ ) of three ROIs 1, 2, and 3 (shown in Figure 5.4) during and after $30 \mathrm{~min}$ of heating.

$T_{1}$ method. According to Figures 5.6 and 5.10, the PRF method has a temperature uncertainty of less than $15 \%$ and the $T_{1}$ method has a temperature uncertainty of above $30 \%$.

\subsection{Evaluation of PRF Method in Ex Vivo Tissue}

In Section 5.1, the PRF method has been experimentally confirmed as a preferred temperature mapping technique when combined with real-time MRI. Despite that the accuracy of this method has been examined on the phantom in Chapter 4, it still needs to be proved under ex vivo conditions. Swine livers and kidneys are employed as the experimental subjects, because minimally invasive or noninvasive surgeries in these two kinds of organs receive many interests in the routine clinical performance. Further, as introduced in Chapter 3, lipid tissue does not show a promising temperature sensitivity to the PRF method $[63,64]$. In this section, experiments are conducted on the tissue with layers of muscle and fat to experimentally confirm this theory. 


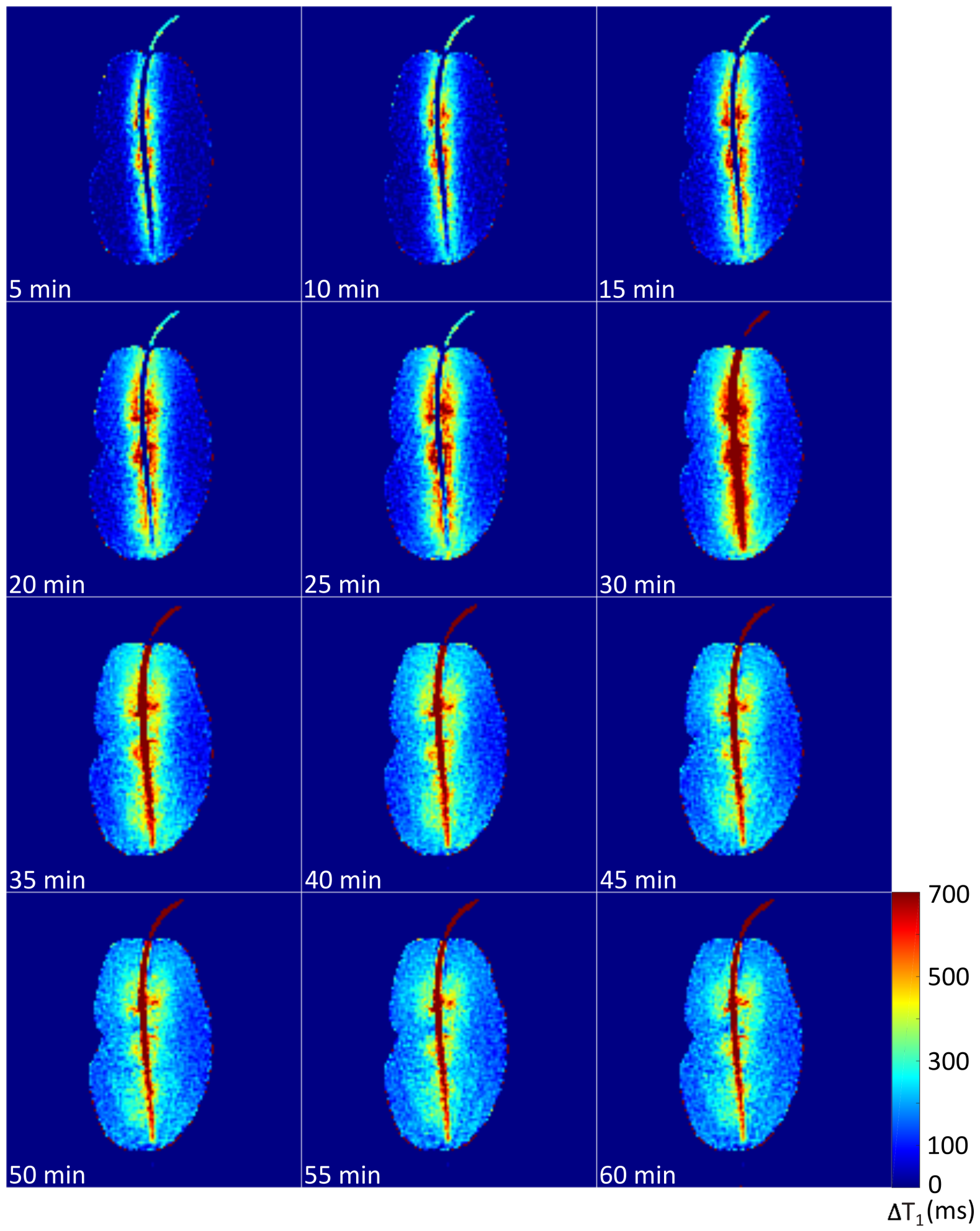

Figure 5.7.: Coronal $T_{1}$ difference maps of the swine kidney obtained every 5 min during and after $30 \mathrm{~min}$ of heating. 


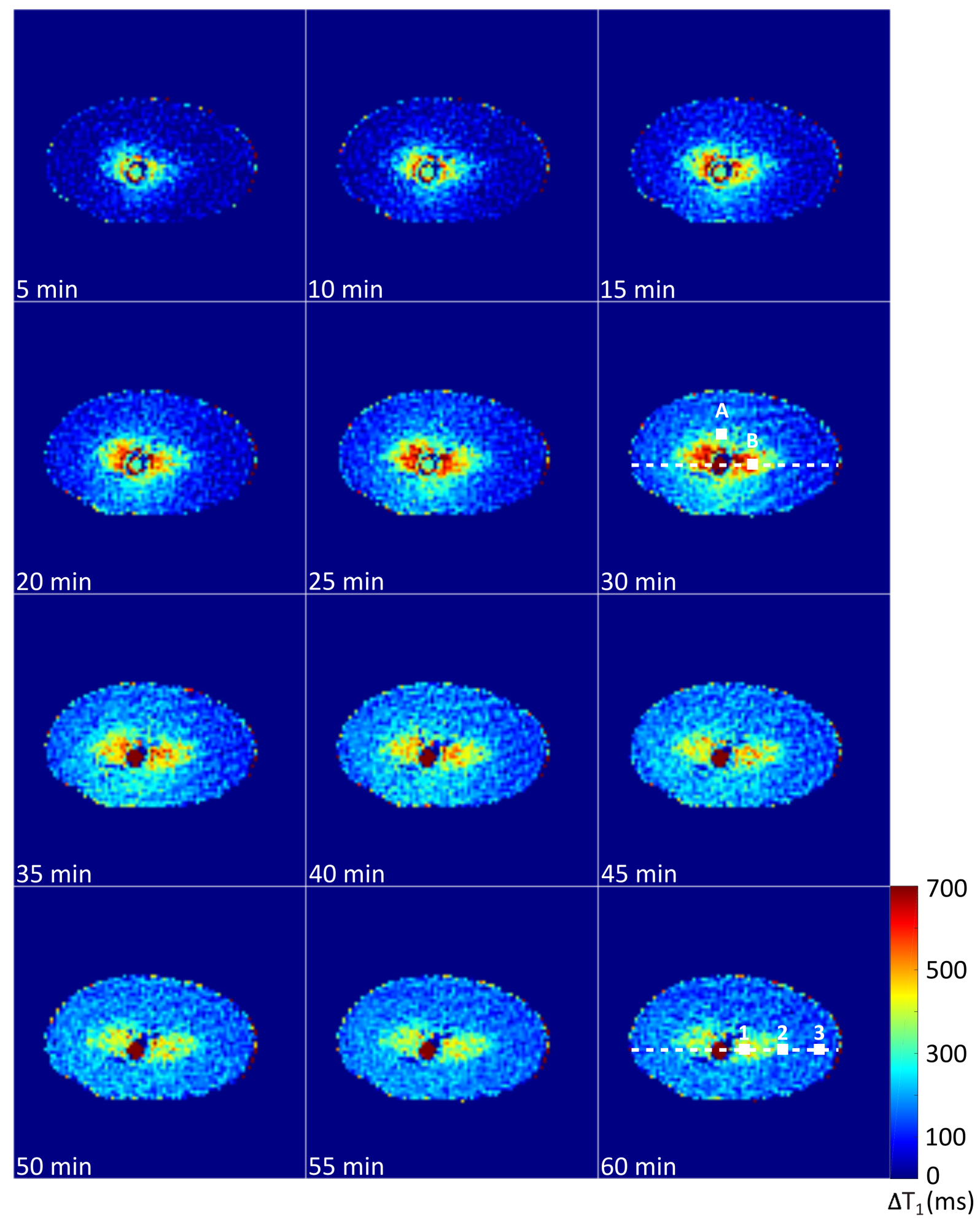

Figure 5.8.: Transversal $T_{1}$ difference maps of the swine kidney obtained every 5 min during and after $30 \mathrm{~min}$ of heating. The $T_{1}$ difference profile along the dashed lines is illustrated in Figure 5.9. ROI $\mathrm{A}$ and $\mathrm{B}$ are used to quantify the $T_{1}$ increase. The $T_{1}$ changes of the three ROIs 1, 2, and 3 (indicated by the three squares) are shown in Figure 5.10. 

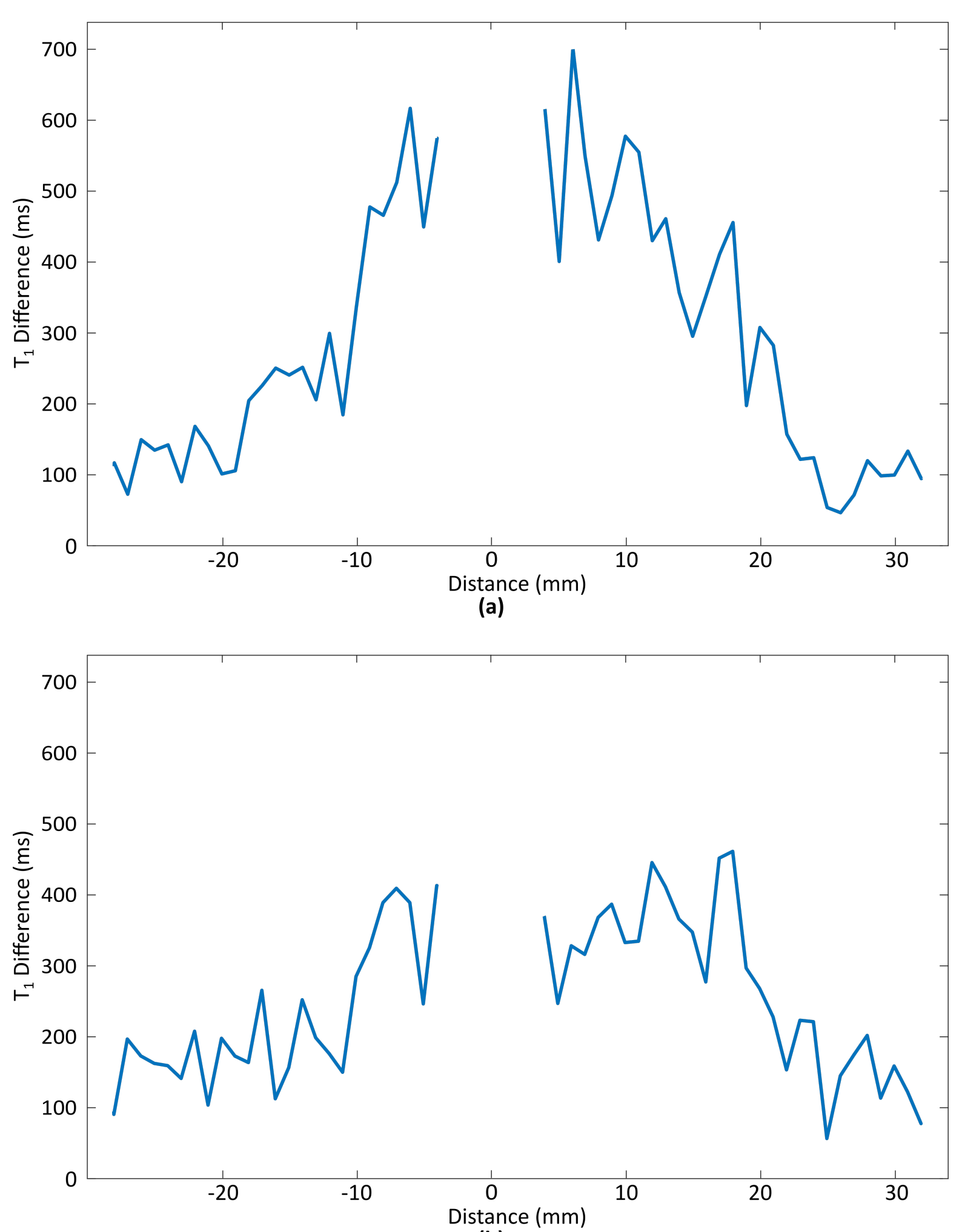

(b)

Figure 5.9.: (a) $T_{1}$ difference profile along the dashed line (indicated in Figure 5.8) obtained after $30 \mathrm{~min}$ of heating. (b) $T_{1}$ difference profile along the dashed line (indicated in Figure 5.8) obtained after 30 min after heating. Values of the central tube are masked. 


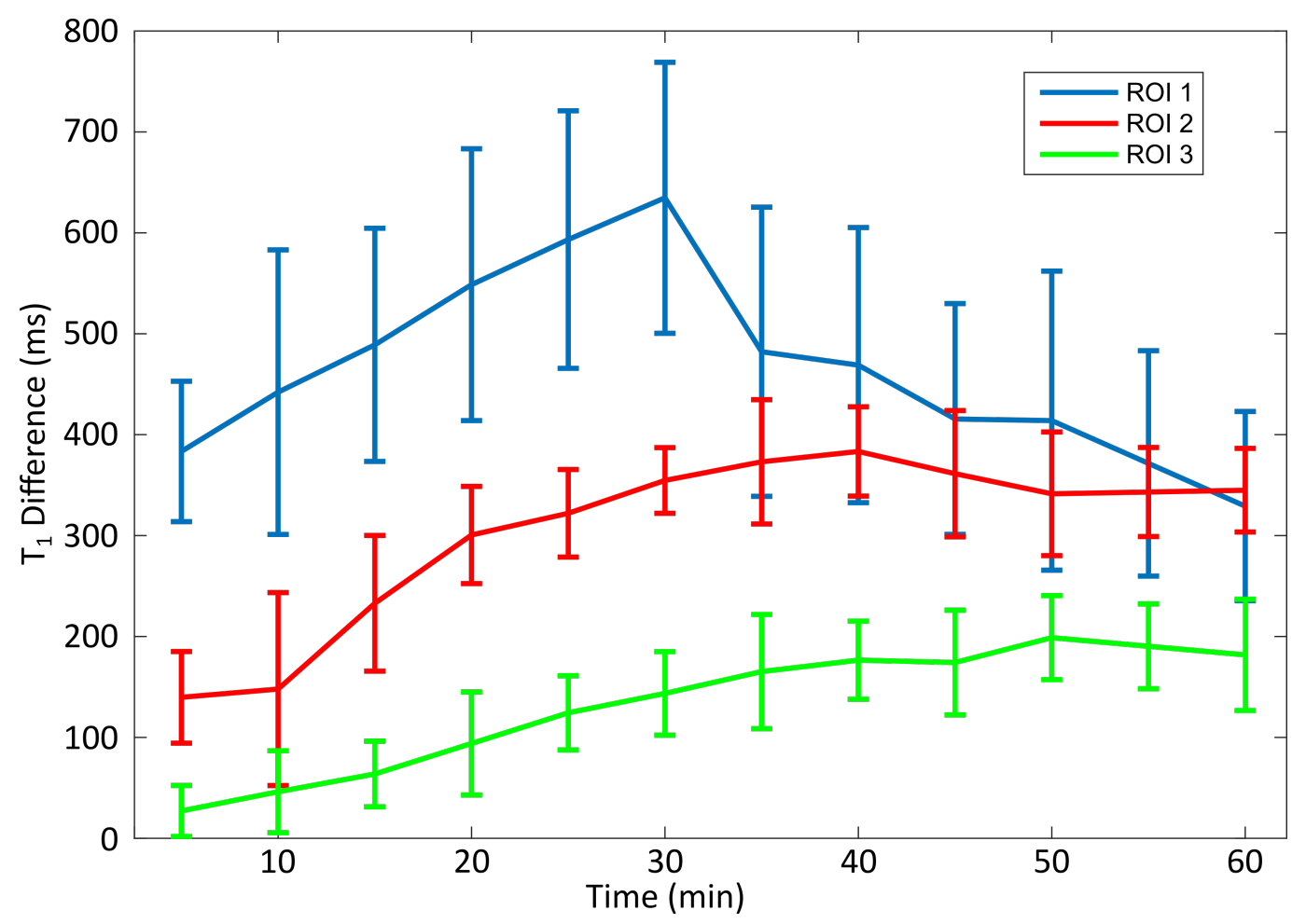

Figure 5.10.: Changes of the $T_{1}$ difference (mean $\pm \mathrm{SD}$ ) of three ROIs 1, 2, and 3 (shown in Figure 5.8) during and after $30 \mathrm{~min}$ of heating.

\subsubsection{Experimental Setup}

\section{Organ Model and Heating Method}

Fresh porcine kidneys, parts of livers and 'Dicke Rippe' purchased from local butchers were used for ex vivo experiments. The tissue preparation procedure and the temperature setting of the cycled water are referred to Section 5.1. Additionally, in order to record the temperature change, a fiber optic sensor was inserted into the kidney and liver parallel to the water tube with a distance of about $5 \mathrm{~mm}$ (Figure 5.11).

\section{MRI Imaging}

Different from phantom studies, a higher spatial resolution $\left(1 \times 1 \times 6 \mathrm{~mm}^{3}\right)$ was used due to the fact that the diameter of tumor in many patients can be on the level of $1 \mathrm{~cm}$ [101] and its spatial information of temperature needs to be better defined. More details about MR parameters are summarized in Table 5.2. Imaging orientations of in the kidney and the liver are demonstrated in Figure 5.11. Slice 1 was used for temperature calculation and the results were compared with the temperature data recorded from the optic sensor in slice 2. Once the pump was turned on, MR sequence was started every 3 min until $30 \mathrm{~min}$. Sagittal view was used as the imaging orientation for 'Dicke Rippe', which cut through the water tube (indicated by the magnitude image in Figure 5.13). Once the 
pump was turned on, the sequence was started every 2 min until 30 min. Subsequently, all the temperature maps are reconstructed as described in Chapter 4.

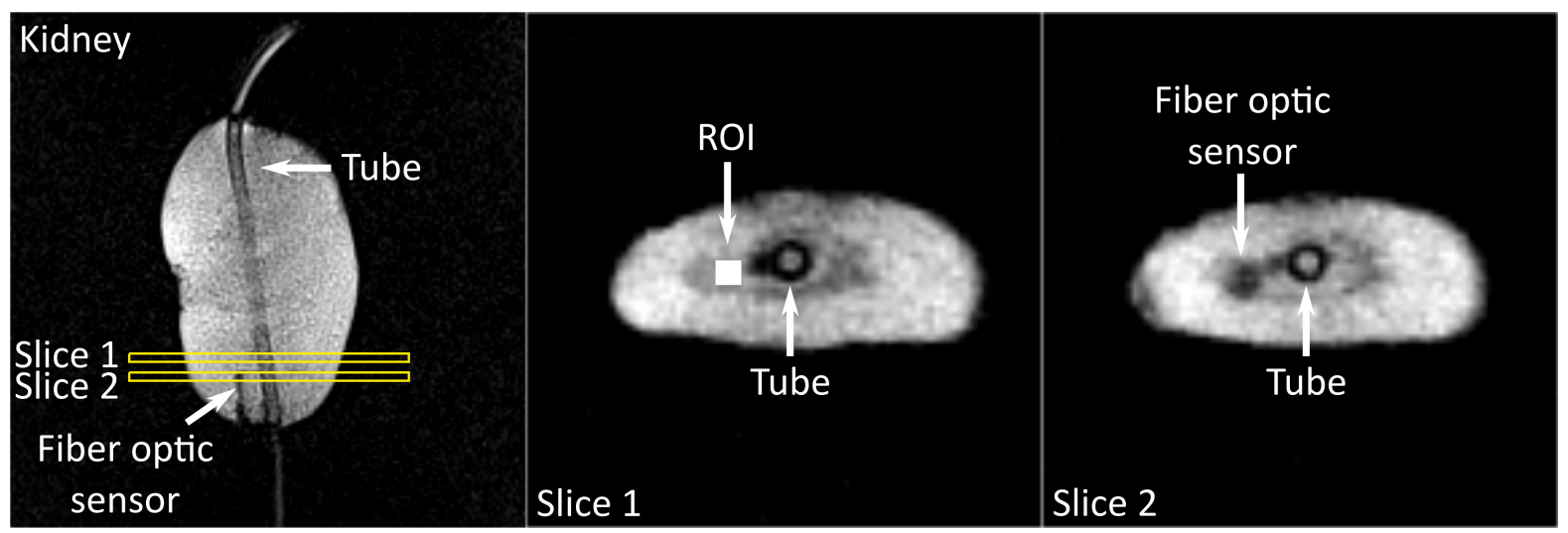

(a)

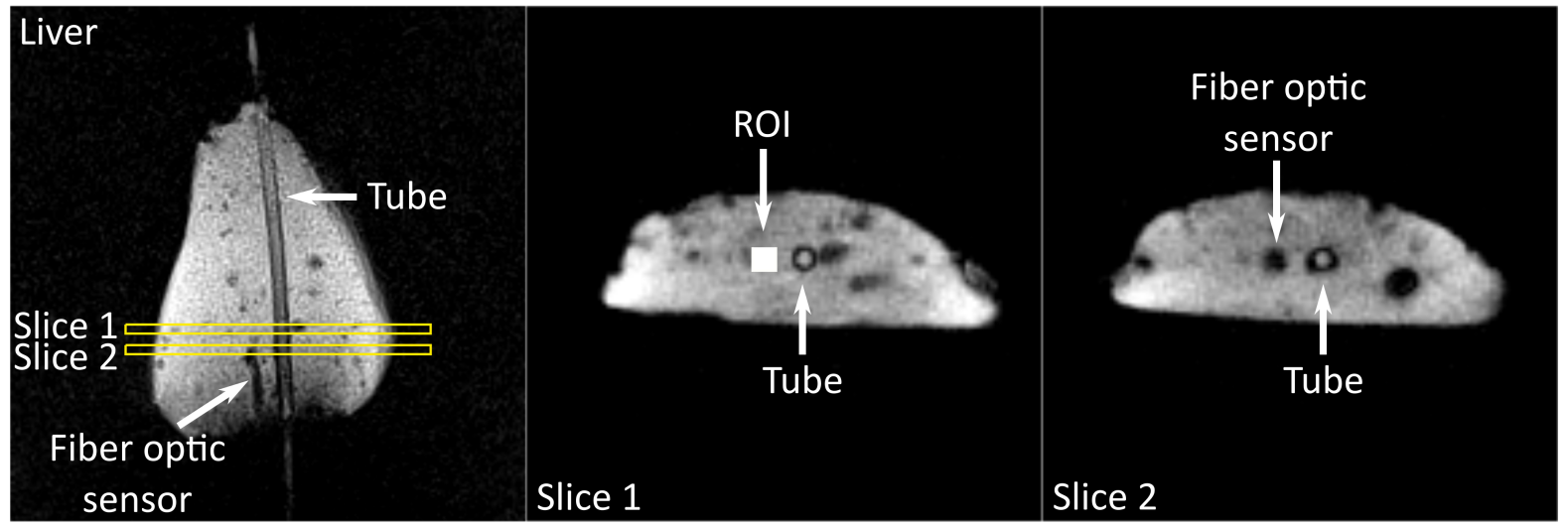

(b)

Figure 5.11.: (a) Left: the coronal MR image of a swine kidney. Middle: a ROI in the kidney medulla (indicated in slice 1) next to the tip of the fiber optic sensor is employed to assess the temperature changes. Right: slice 2 exhibits the location of the tip of the fiber optic sensor. (b) Left: the coronal MR image of a part of a swine liver. Middle: a ROI (indicated in slice 1) next to the tip of the fiber optic sensor is employed to assess the temperature changes. Right: slice 2 exhibits the location of the tip of the fiber optic sensor.

Table 5.2.: MR parameters used for PRF thermometry on ex vivo tissue.

\begin{tabular}{cc|cc}
\hline Sequence & Radial FLASH & FOV & $128 \times 128 \mathrm{~mm}^{2}$ \\
\hline TE/TR & $10 / 11.8 \mathrm{~ms}$ & In-plane resolution & $1 \times 1 \mathrm{~mm}^{2}$ \\
\hline Flip angle & $10^{\circ}$ & Number of spokes & 17 per frame \\
\hline Slice thickness & $6 \mathrm{~mm}$ & Temporal resolution & $200 \mathrm{~ms}$ \\
\hline
\end{tabular}




\subsubsection{Results}

As shown in Figure 5.11, according to the location of the temperature sensor, a ROI $(4 \times 4$ pixels) in the slice 1 is selected for quantitative evaluation of the PRF method for both of the tissue types. Figure 5.12 compares the results of the chosen ROI (mean \pm SD) and the true temperature differences recorded by the fiber optic sensor. In general, the calculated values with the PRF method match the recorded data. In both liver and kidney, the mean values of the temperature difference are slightly higher than the recorded temperature data in the beginning of the experiment, however, these difference are all smaller than the standard deviations. After 15 min of heating, the computed mean values are identical with the recorded data.

Muscle and fat in 'Dicke Rippe' are indicated by the arrows in the magnitude image acquired by PRF sequence in Figure 5.13. Lipid has a lower signal intensity compared to muscle due to its faster $\mathrm{T}_{2}^{*}$ decay [102]. Figure 5.13 shows the temperature distribution during heating, which were derived based on the PRF theory in water tissue. Different from layers of muscle, calculated temperature differences of fat are almost unnoticeable. In addition, temperature change of two ROIs are depicted in Figure 5.14, these ROIs are selected from regions of muscle and fat, respectively and share the same distance to the heating tube ( $8 \mathrm{~mm}$, shown in the last map in Figure 5.13). The temperature in muscle increases during the heating procedure, in contrast, the temperature change in fat stays around zero. Due to the fact that low signal intensities are acquired in the fat region, a higher SD is obtained in the ROI from fat $\left(1.8^{\circ} \mathrm{C}\right)$ than that from muscle $\left(1.2^{\circ} \mathrm{C}\right)$.

\subsubsection{Discussion}

In this section, phase errors induced by the magnetic field drift during the experiment are ignored, because the total measuring time $(30 \mathrm{~min})$ is much shorter than that in phantom studies (2 hours). Figure 5.12 demonstrates that temperature data acquired from real-time MRI match the true temperature values quite well even without using the reference phantoms for the field drift correction, which offers an opportunity to only acquire reasonable FOV without extra room for reference phantoms.

As shown in Figure 5.12, the SDs of the selected ROI in ex vivo studies $\left(1.5^{\circ} \mathrm{C}\right)$ is higher than that in phantom studies (less than $1^{\circ} \mathrm{C}$ ). This could be explained by the following reason. A high spatial resolution is employed data sampling which leads to a decrease of SNR in the complex anatomical images and thus, higher SDs are obtained in the temperature maps. This SD acquired in ex vivo is still acceptable, because the temperature is usually increased by at least $15{ }^{\circ} \mathrm{C}$ during tissue coagulation surgeries [49]. 


\subsubsection{Summary}

In this section, it has been confirmed that the PRF method can provide accurate temperature changes when combined with real-time MRI in swine liver and kidney. All the calculated mean values and standard deviations show nice agreements with the temperature acquired from the fiber optic sensor. Additionally, a high in-plane resolution is performed for data acquisition which brings the experiments much closer to clinical condition.

The measurement conducted on 'Dicke Rippe' experimentally demonstrates that the PRF method is not suitable in adipose tissue. Because minimally invasive or noninvasive surgery does not pay too many attention on tumor in fat [103], the PRF method can still be considered as the optimal technique for MR thermometry. 
5. Real-time MR Thermometry - Ex Vivo Tissue Studies with Global Heating

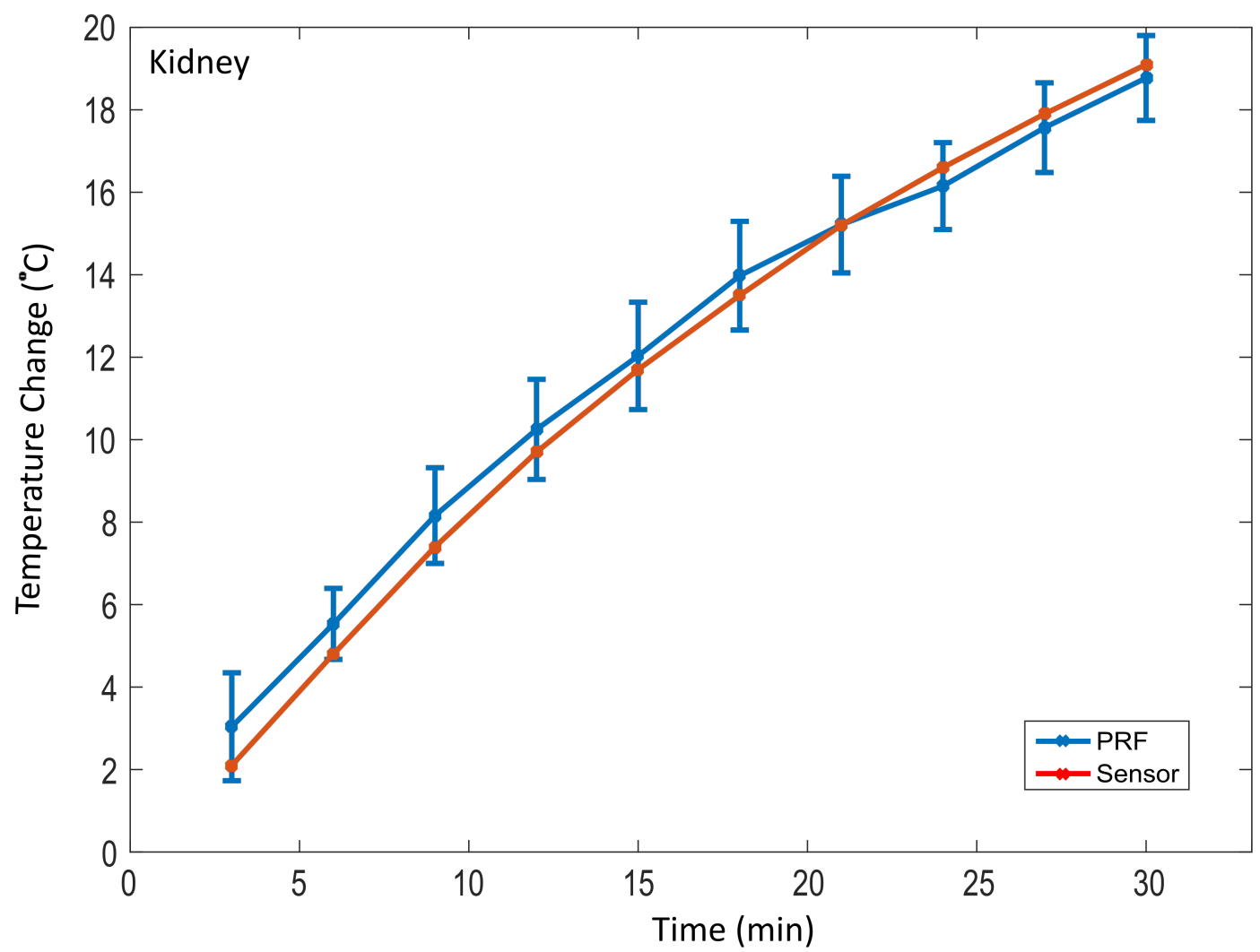

(a)

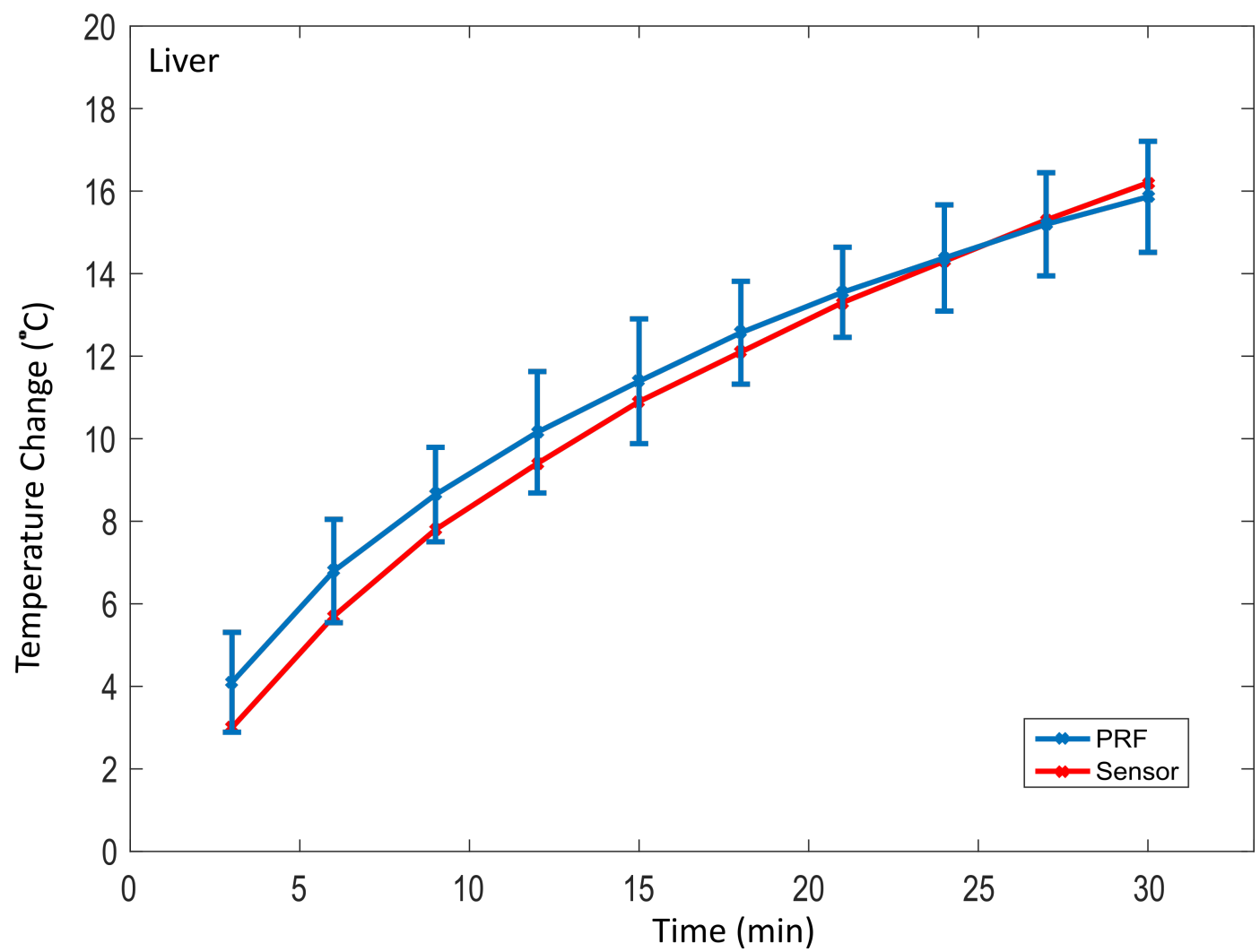

(b)

Figure 5.12.: Comparison between the temperature differences calculated with the PRF method and the true temperature recorded with the fiber optic sensor. 


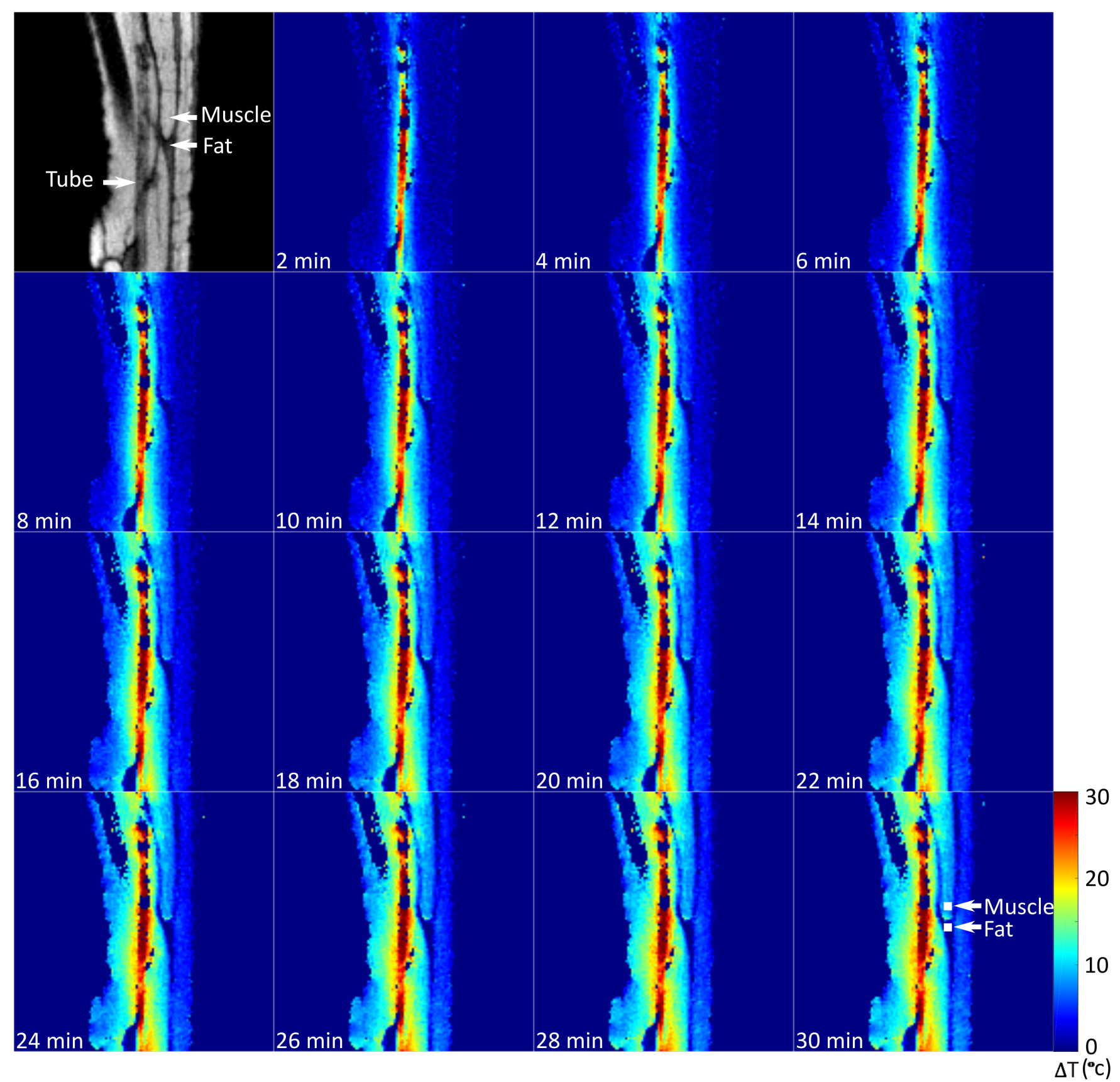

Figure 5.13.: Sagittal magnitude and relative temperature difference maps of fat and muscle tissue obtained every 2 min during 30 min of heating. The temperature evolution of the ROIs placed in muscle and fat tissue are shown in Figure 5.14. 
5. Real-time MR Thermometry - Ex Vivo Tissue Studies with Global Heating

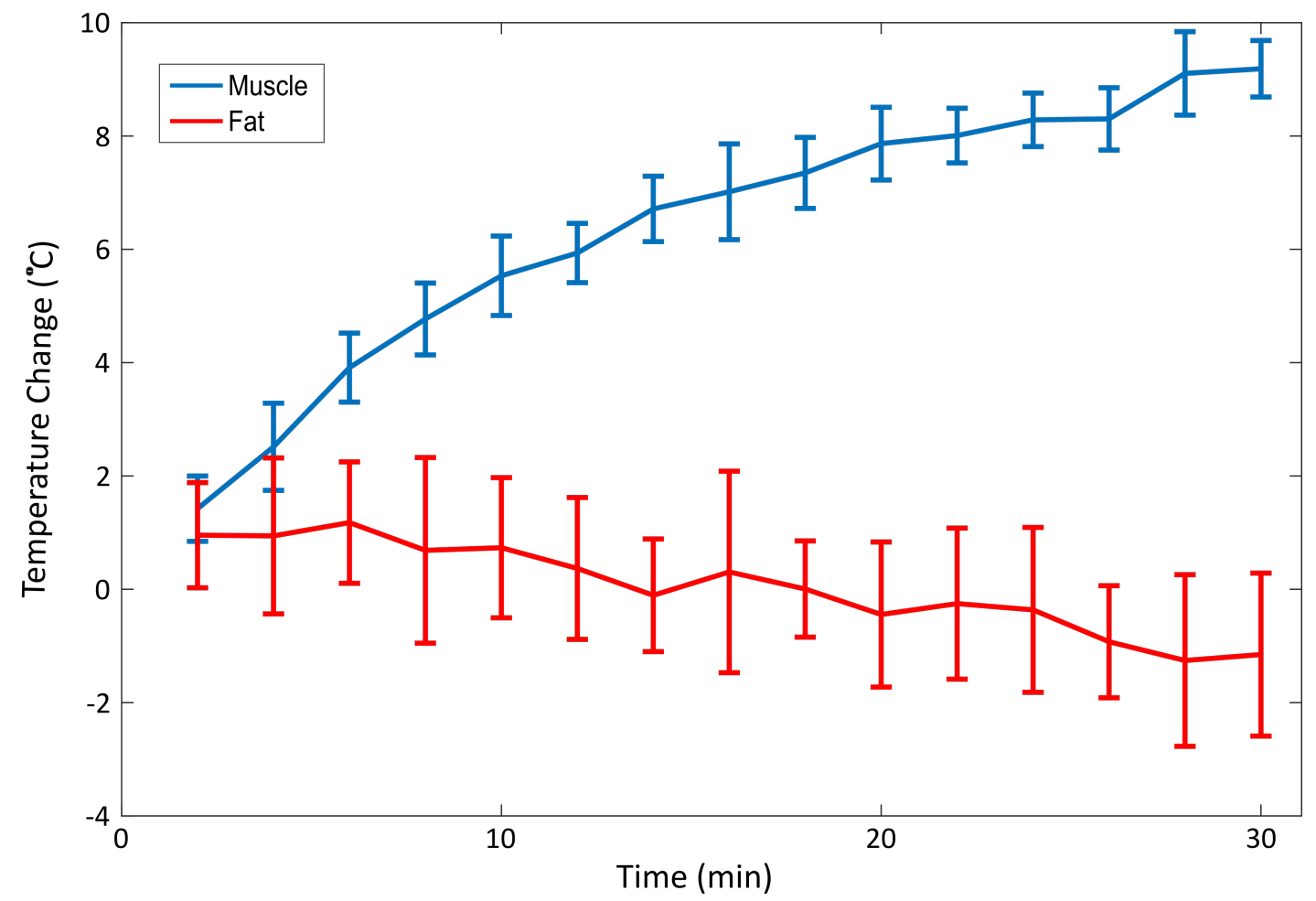

Figure 5.14.: The temperature evolution of muscle (blue) and fat (red) during $30 \mathrm{~min}$ of heating. Please note that both ROIs that have the same distance to the central water tube $(8 \mathrm{~mm})$. 


\section{Real-time MR Thermometry - Ex Vivo Tissue Studies with Focal Heating}

In the previous chapters, the PRF method has been confirmed as the preferred technique for MR thermometry when combined with real-time MRI. This method has been experimentally proven that it can provide accurate temperature changes in both phantoms and ex vivo tissues by means of water tube heating. For the purpose of better mimicking the procedure during thermal therapy, in this chapter, MRI experiments are performed with the use of a diode-pumped laser system to introduce well-defined focal lesions in the target tissues. Temperature changes are calculated and analyzed under different laser operational modes in both cortex and medulla of swine kidneys.

\subsection{MRI Sequence Optimization}

For the purpose of providing more anatomical and temperature information, the PRF sequence is optimized under two aspects. On one hand, in order to introduce laser energy into the kidney and to have a temperature control near the heated region, as illustrated in Figure 6.1 (a), the laser fiber and the fiber optic sensor are parallel inserted about $1 \mathrm{~cm}$ into the cortex of the kidney and a high spatial resolution is required to locate these two fibers in the anatomical images. Therefore, a in-plane resolution of $0.5 \times 0.5 \mathrm{~mm}^{2}$ is employed and a slice thickness of $3 \mathrm{~mm}$ is achieved by including more side lobes of the $\mathrm{RF}$ excitation pulse. On the other hand, because images acquired with a TE of $10 \mathrm{~ms}$ are used for temperature calculation, there is still enough time to include another echo with a shorter TE (2.64 ms) into the sequence that can provide another image with better anatomical details and SNR without sacrificing imaging temporal resolution. Because there is no flow in the ex vivo tissue, the flow compensation gradient is not employed in the MR sequence. More sequence details are summarized in Table 6.1. As shown in Figure 6.1 (b), $\mathrm{T}_{2}^{*}$ decay contributes to the signal loss in the magnitude images obtained at different TE. 

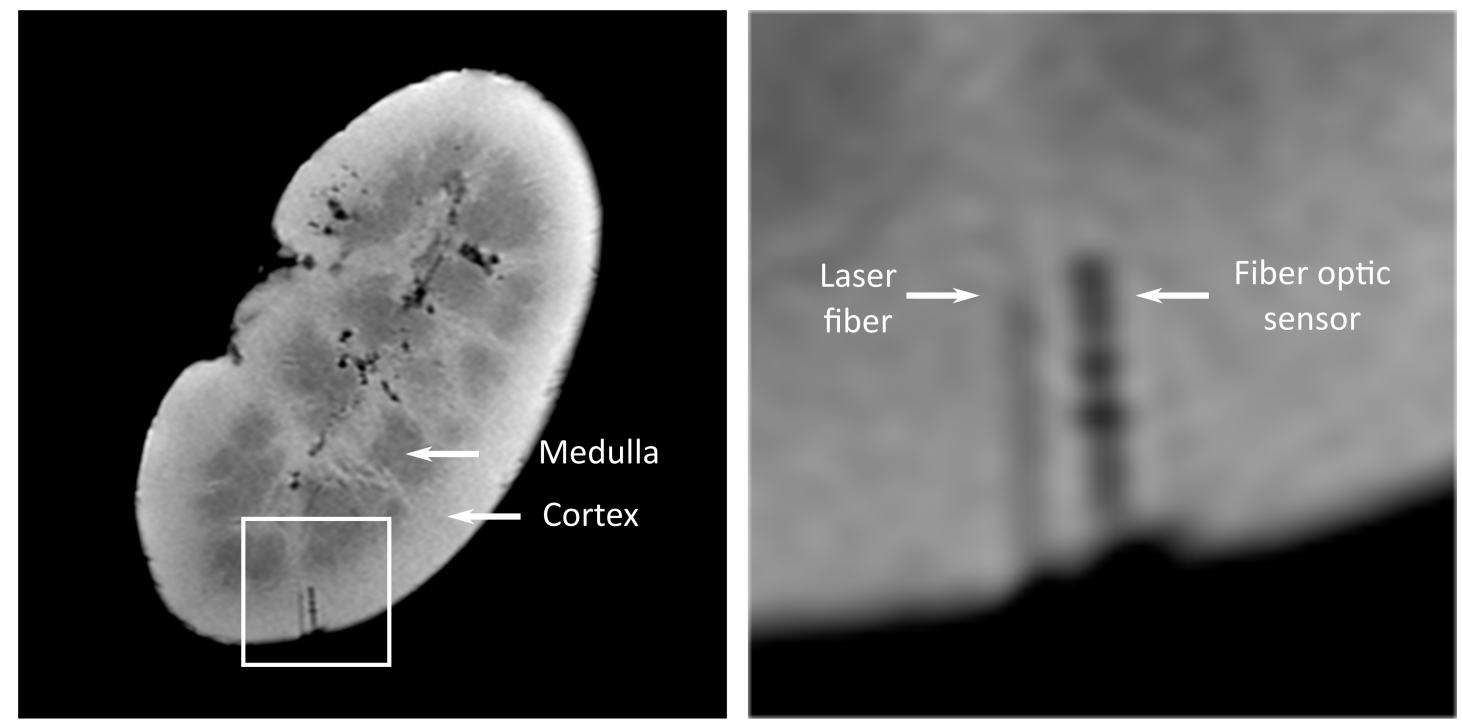

(a)

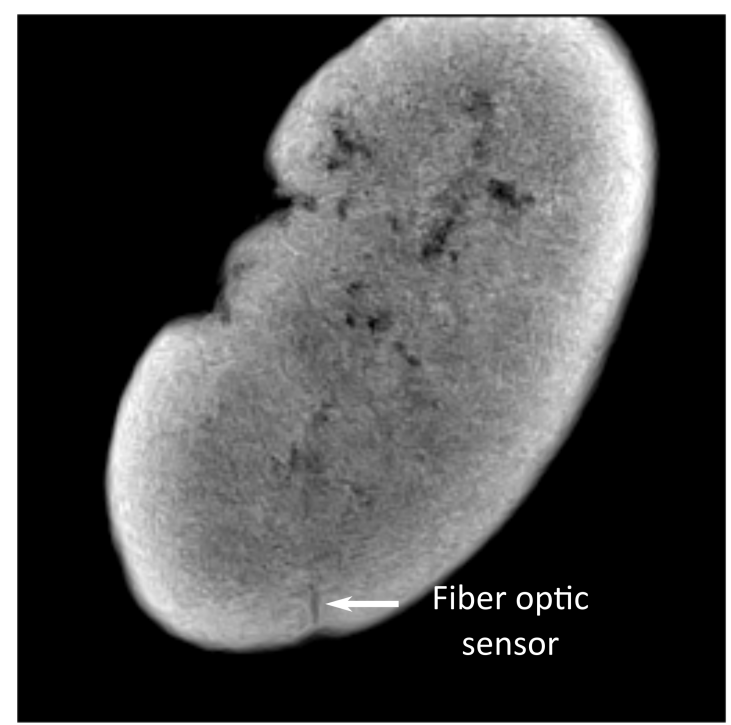

$\mathrm{TE}=2.64 \mathrm{~ms}$

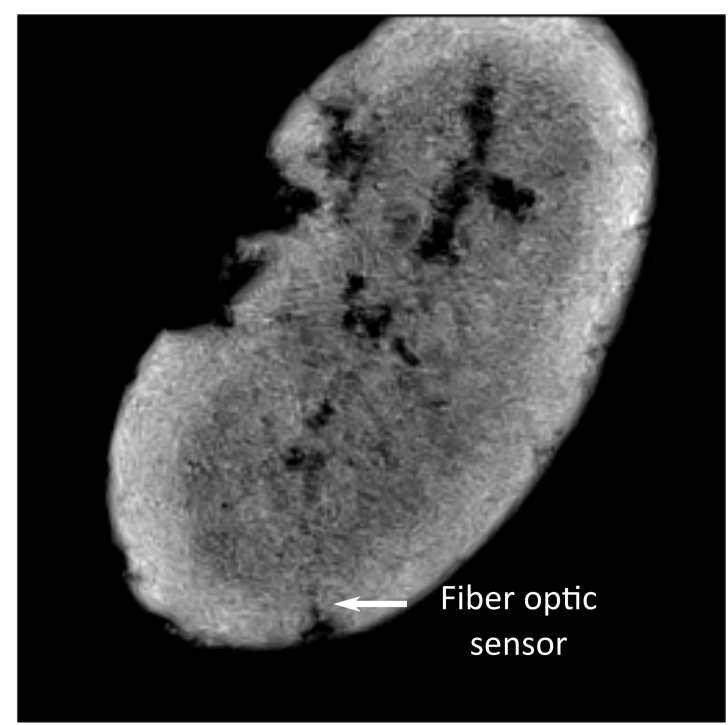

$\mathrm{TE}=10 \mathrm{~ms}$

(b)

Figure 6.1.: (a) A coronal MR image of a kidney (left) and a magnified ROI (right) acquired by high spatial resolution $T_{1}$ weighted $3 \mathrm{D}$ MRI $\left(0.3 \times 0.3 \times 0.3 \mathrm{~mm}^{3}\right)$ for slice selection. The laser fiber and the fiber optic sensor are inserted into the cortex. (b) MR images of the kidney obtained with the MR thermometry sequence using two echoes. The MR parameters of the used sequence are summarized in Table 6.1. 
Table 6.1.: MR parameters for PRF thermometry during laser heating.

\begin{tabular}{cc|cc}
\hline Sequence & Radial FLASH & FOV & $128 \times 128 \mathrm{~mm}^{2}$ \\
\hline $\mathrm{TE}_{1} / \mathrm{TE}_{2} / \mathrm{TR}$ & $2.64 / 10 / 11.9 \mathrm{~ms}$ & In-plane resolution & $0.5 \times 0.5 \mathrm{~mm}^{2}$ \\
\hline Flip angle & $10^{\circ}$ & Number of spokes & 17 per frame \\
\hline Slice thickness & $3 \mathrm{~mm}$ & Temporal resolution & $200 \mathrm{~ms}$ \\
\hline
\end{tabular}

\subsection{Dynamic Temperature Monitoring during Laser Heating}

As introduced in Chapter 3, a $2 \mu \mathrm{m}$ wavelength laser system, which has a penetration depth of about $1 \mathrm{~mm}$ in aqueous tissue [104], is employed in the present work. In order to avoid undesired tissue damage by the high power density within the penetration depth, only the pulsed or the single pulse mode is used in the experiments. Temperature maps are calculated by using the data acquired at $\mathrm{TE}=10 \mathrm{~ms}$. MR images acquired before laser heating are adopted as the baseline information for temperature calculation.

\subsubsection{Laser Power \& Temperature}

\section{Experiment Procedure}

As described in Section 6.1, the laser fiber and the fiber optic sensor were inserted into the kidney cortex for about $1 \mathrm{~cm}$. Three different laser parameters were applied in the experiments to introduce different energy deposition in the kidney (similar laser output power has been used in [105] and all the parameters are summarized in Table 6.2). In order to eliminate artifacts caused by the tissue coagulation and the residue temperature increases from previous experiments, for each investigation, the laser fiber and the fiber optic sensor inserted into a new location of the kidney.

For the purpose of monitoring the temperature changes during and after the heating procedure, continuous MR thermometry measurement was started a few seconds before laser heating until $1 \mathrm{~min}$ after turning off the laser. 
Table 6.2.: Laser protocols used for investigating temperature variations under different output power.

\begin{tabular}{lcccccc}
\hline & Mode & $\begin{array}{c}\text { Output } \\
\text { power }\end{array}$ & $\begin{array}{c}\text { Repetition } \\
\text { rate }\end{array}$ & $\begin{array}{c}\text { Pulse } \\
\text { duration }\end{array}$ & $\begin{array}{c}\text { Applied } \\
\text { time }\end{array}$ & $\begin{array}{c}\text { Output } \\
\text { energy }\end{array}$ \\
\hline Experiment 1 & Pulsed & $10 \mathrm{~W}$ & $0.5 \mathrm{~Hz}$ & $100 \mathrm{~ms}$ & $2 \mathrm{~min}$ & $60 \mathrm{~J}$ \\
\hline Experiment 2 & Pulsed & $20 \mathrm{~W}$ & $0.5 \mathrm{~Hz}$ & $100 \mathrm{~ms}$ & $2 \mathrm{~min}$ & $120 \mathrm{~J}$ \\
\hline Experiment 3 & Pulsed & $30 \mathrm{~W}$ & $0.5 \mathrm{~Hz}$ & $100 \mathrm{~ms}$ & $2 \mathrm{~min}$ & $180 \mathrm{~J}$ \\
\hline
\end{tabular}

\section{Results}

Figures 6.2, 6.3 and 6.4 demonstrate the results obtained with a laser output power of $10 \mathrm{~W}, 20 \mathrm{~W}$, and $30 \mathrm{~W}$, respectively. The temperature maps demonstrate that focal heating is achieved in the three experiments. The temperature increases in the target area during the heating period and drops after switching off the laser system. As demonstrated in Figure 6.5, using the same heating time (120 s), increased temperature differences can be observed in the core area with increased output power and the diameters of the effected area remain about $14 \mathrm{~mm}$. This is in line with the assumption that the thermal diffusivity is constant within the kidney cortex. In the temperature map acquired with the laser power of $30 \mathrm{~W}$ (Figure 6.4), a 'black hole' can be observed in the central part of the heated region and the temperature of the tissue along the laser fiber is also increased (indicated by the arrow). According to the locations of the chosen ROIs, which are $2 \mathrm{~mm}$ apart from the laser fiber (indicated in Figures 6.2, 6.3 and 6.4), the temperature changes are shown in Figure 6.6. For the three experiments, the temperatures show similar changes for the first $20 \mathrm{~s}$ of heating and reach temperature differences of $10{ }^{\circ} \mathrm{C}$, $13^{\circ} \mathrm{C}$ and $22^{\circ} \mathrm{C}$ after $120 \mathrm{~s}$ of heating, respectively. Similar to the results acquired after water tube heating, the temperature differences drop to less than $5{ }^{\circ} \mathrm{C}$ after switching off the laser $60 \mathrm{~s}$. In the magnitude images of the three experiments, the signal intensity decreases near the the tip of the laser fiber during heating and recovers after the heating procedure. The size of this 'black hole' increases with TE and laser output power. More investigations concerning this 'black hole' are performed in the following section.

\subsubsection{Determination of 'Black Hole'}

As shown in Section 6.2.1, a 'black hole' can be observed in magnitude images during laser heating or in temperature maps under high laser power (30 W, Figure 6.4). Further experiments are necessary to clarify the origin of this observation. 


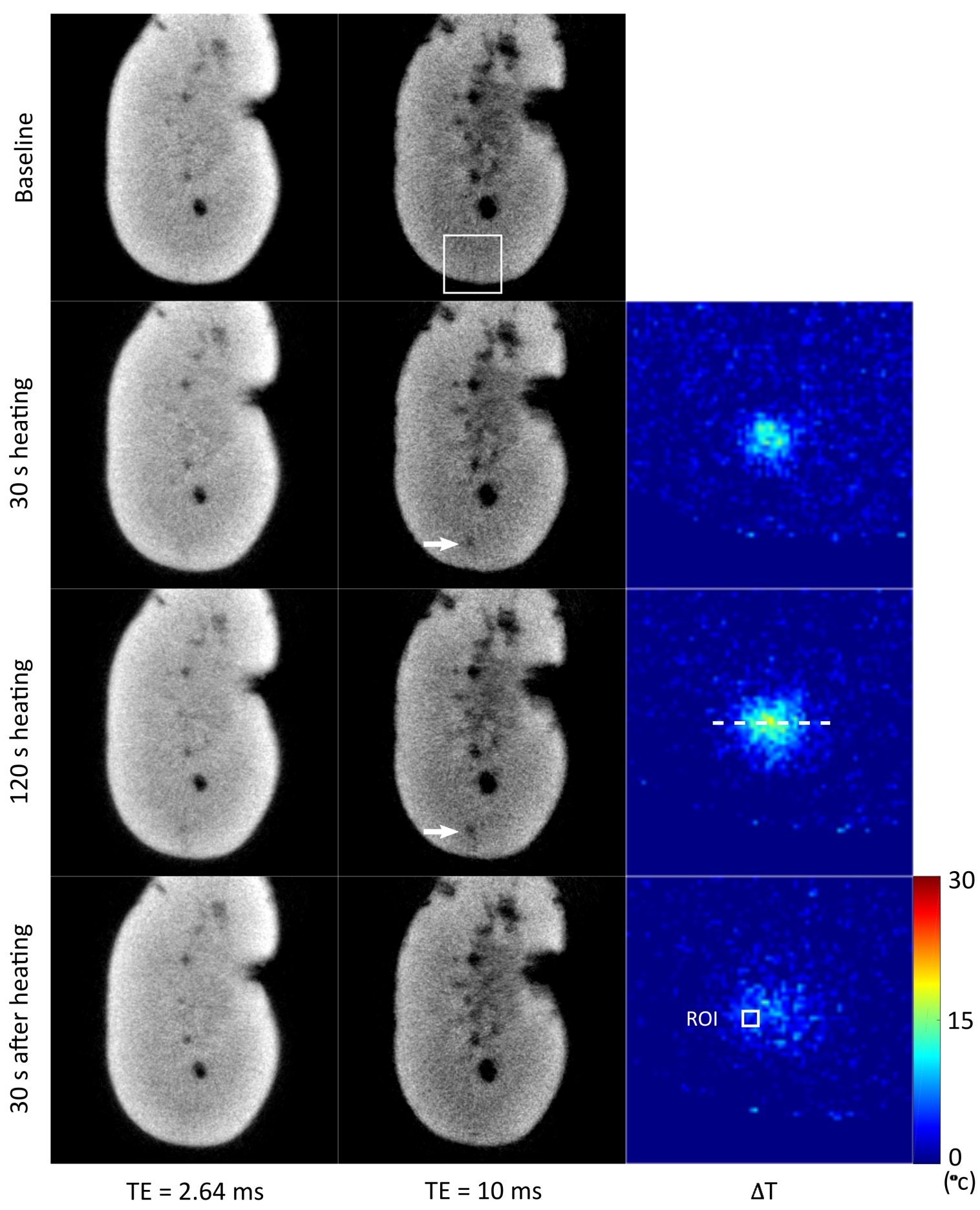

Figure 6.2.: MR magnitude images of a kidney before and after $120 \mathrm{~s}$ of laser pulsing in the cortex (parameter summarized in Table 6.2 (Experiment 1), output power and energy are $10 \mathrm{~W}$ and $60 \mathrm{~J}$, respectively) obtained with a $\mathrm{TE}=2.64 \mathrm{~ms}$ (left column) and TE $=10 \mathrm{~ms}$ (middle column). Magnified (as indicated by the box) temperature difference maps (right column) were calculated from the corresponding images obtained with TE $=10 \mathrm{~ms}$. Arrows indicate signal loss during laser pulsing. The temperature profile along the dashed line is illustrated in Figure 6.5. The temperature change of a ROI next to the tip of the laser fiber is shown in Figure 6.6. 


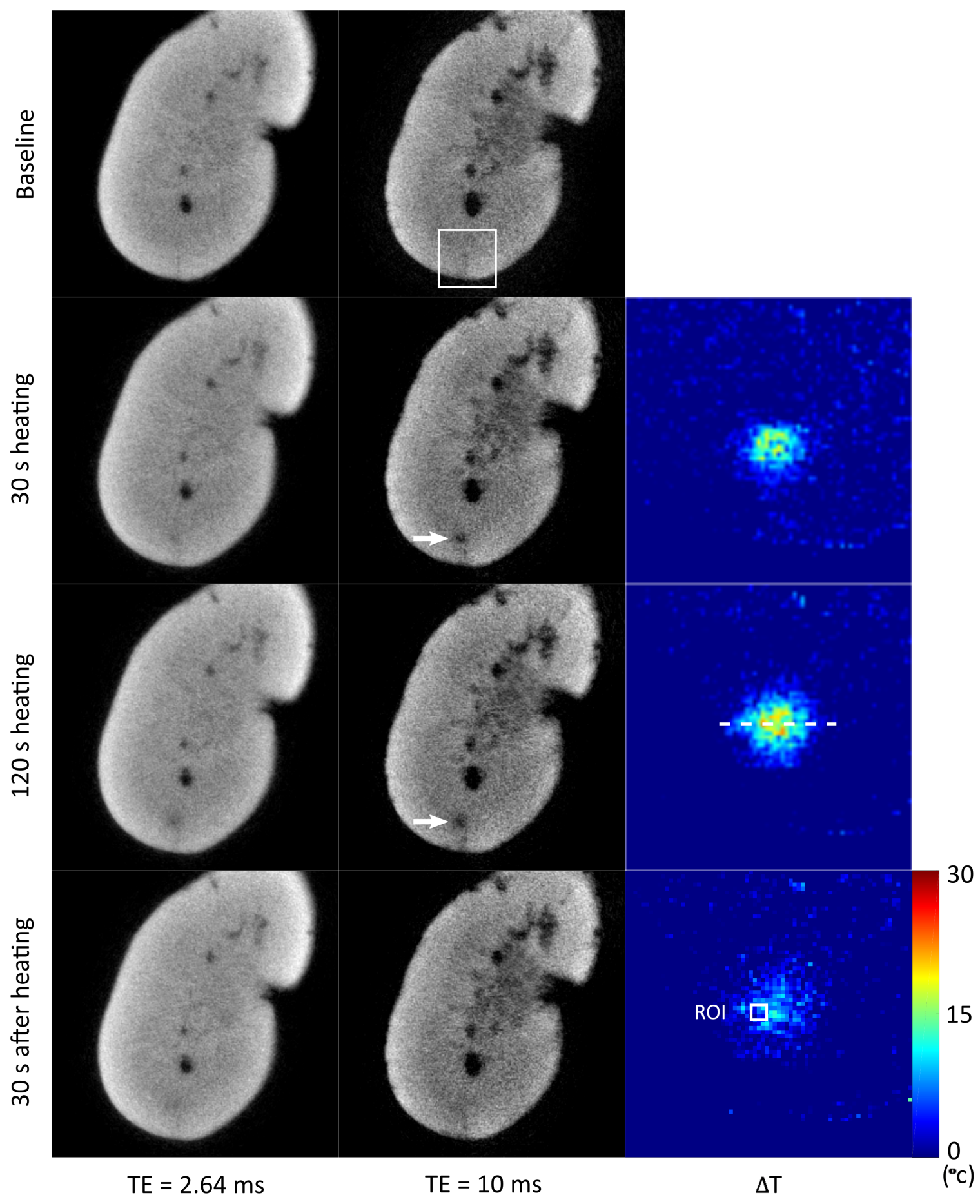

Figure 6.3.: MR magnitude images of a kidney before and after $120 \mathrm{~s}$ of laser pulsing in the cortex (parameter summarized in Table 6.2 (Experiment 2), output power and energy are $20 \mathrm{~W}$ and $120 \mathrm{~J}$, respectively) obtained with a $\mathrm{TE}=2.64 \mathrm{~ms}$ (left column) and TE $=10 \mathrm{~ms}$ (middle column). Magnified (as indicated by the box) temperature difference maps (right column) were calculated from the corresponding images obtained with TE $=10 \mathrm{~ms}$. Arrows indicate signal loss during laser pulsing. The temperature profile along the dashed line is illustrated in Figure 6.5. The temperature change of a ROI next to the tip of the laser fiber is shown in Figure 6.6. 


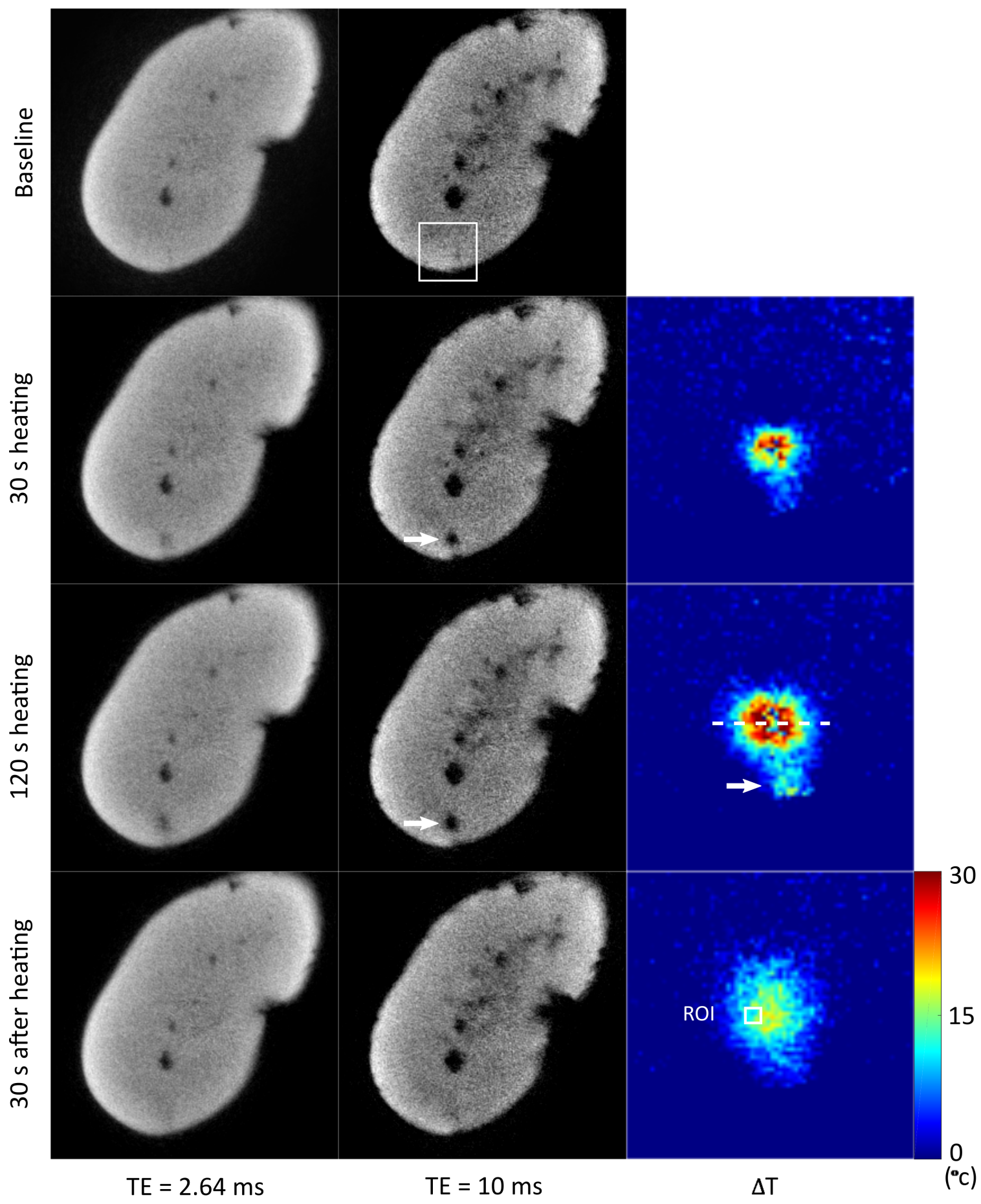

Figure 6.4.: MR magnitude images of a kidney before and after $120 \mathrm{~s}$ of laser pulsing in the cortex (parameter summarized in Table 6.2 (Experiment 3), output power and energy are $30 \mathrm{~W}$ and $180 \mathrm{~J}$, respectively) obtained with a $\mathrm{TE}=2.64 \mathrm{~ms}$ (left column) and TE $=10 \mathrm{~ms}$ (middle column). Magnified (as indicated by the box) temperature difference maps (right column) were calculated from the corresponding images obtained with TE $=10 \mathrm{~ms}$. Arrows indicate signal loss during laser pulsing. The temperature profile along the dashed line is illustrated in Figure 6.5. The temperature change of a ROI next to the tip of the laser fiber is shown in Figure 6.6. 


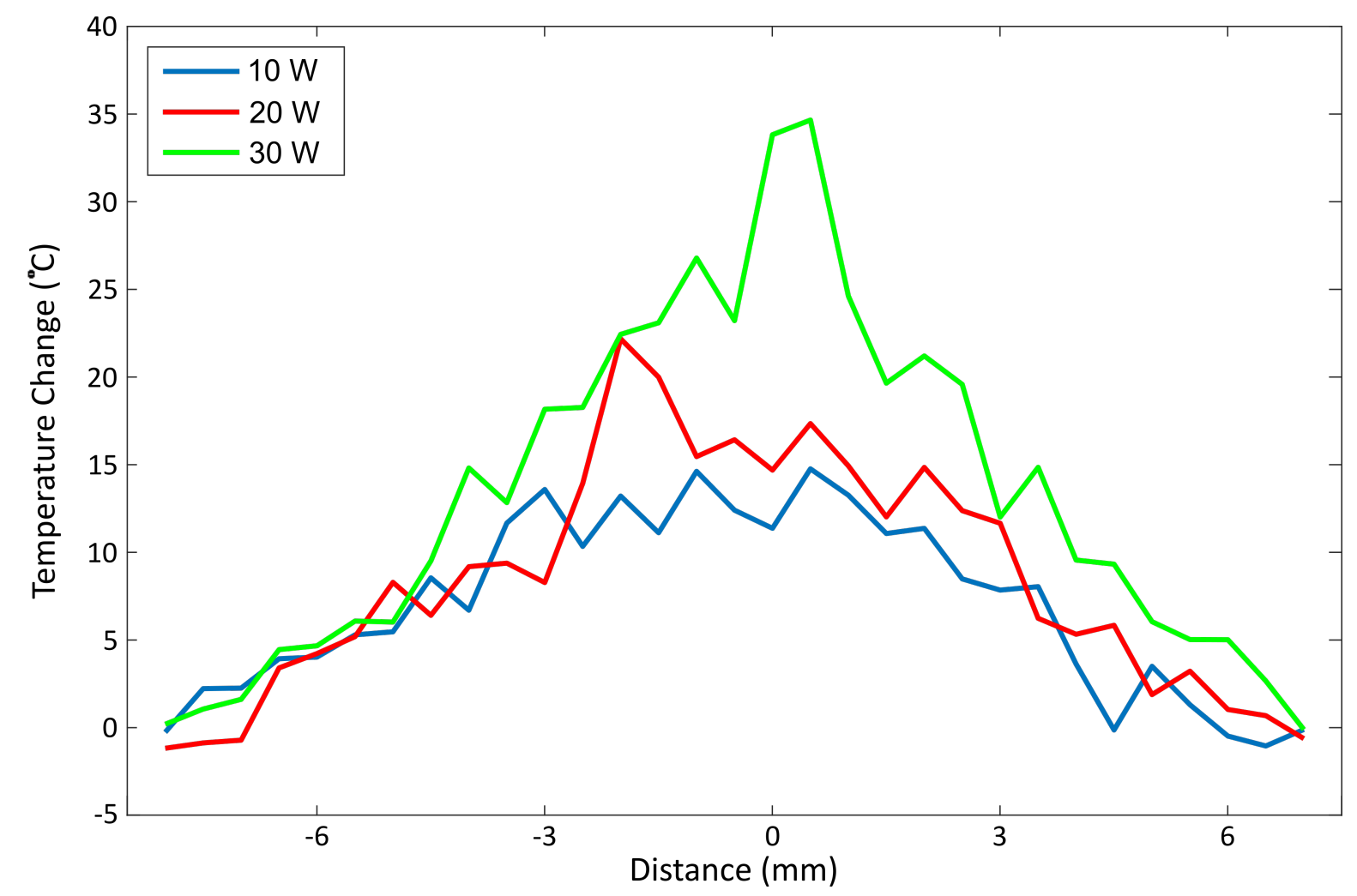

Figure 6.5.: Temperature profile along the dashed line (indicated in Figures 6.2, 6.3 and 6.4) obtained after $120 \mathrm{~s}$ of laser pulsing with different output power and energy.

\section{Methods and Materials}

As observed in Figure 6.4, an output energy of $180 \mathrm{~J}$ certainly results in a lesion. Therefore laser parameters are set to: pulsed mode, output power $=3 \mathrm{~W}$, repetition rate $=10 \mathrm{~Hz}$, pulse duration $=20 \mathrm{~ms}$ and output energy $=234 \mathrm{~J}$. Proton density weighted 3D MRI was performed before, during and after laser heating. The following sequence parameters were employed: $\mathrm{TE}=4.66 \mathrm{~ms}, \mathrm{TR}=11 \mathrm{~ms}$, flip angle $=5^{\circ}$, voxel size $=0.5 \times 0.5 \times 0.5 \mathrm{~mm}^{3}$, number of scan average $=2$ and total acquisition time $=330 \mathrm{~s}$. A transverse slice of the 3D data sets (Figure 6.7), which contains the laser fiber and temperature sensor, was selected for data analysis.

\section{Results}

Proton density weighted images and the photo of the corresponding post-treatment kidney section are shown in Figure 6.7. Compared with the image obtained before heating, a 'black hole' with a diameter of about $5 \mathrm{~mm}$ appears in the image acquired during the heating procedure. In the two images acquired after heating, the 'black hole' remains, while its diameter is reduced to about $2.5 \mathrm{~mm}$. In the post-treatment photo, tissue near the fiber tip is ablated (diameter of about $1 \mathrm{~mm}$ ) and a most likely deproteinized lesion with a diameter of about $3 \mathrm{~mm}$ can be observed. The location of the temperature sensor is illustrated in the preheating image. Due to the presence of the 'black hole', the sensor 


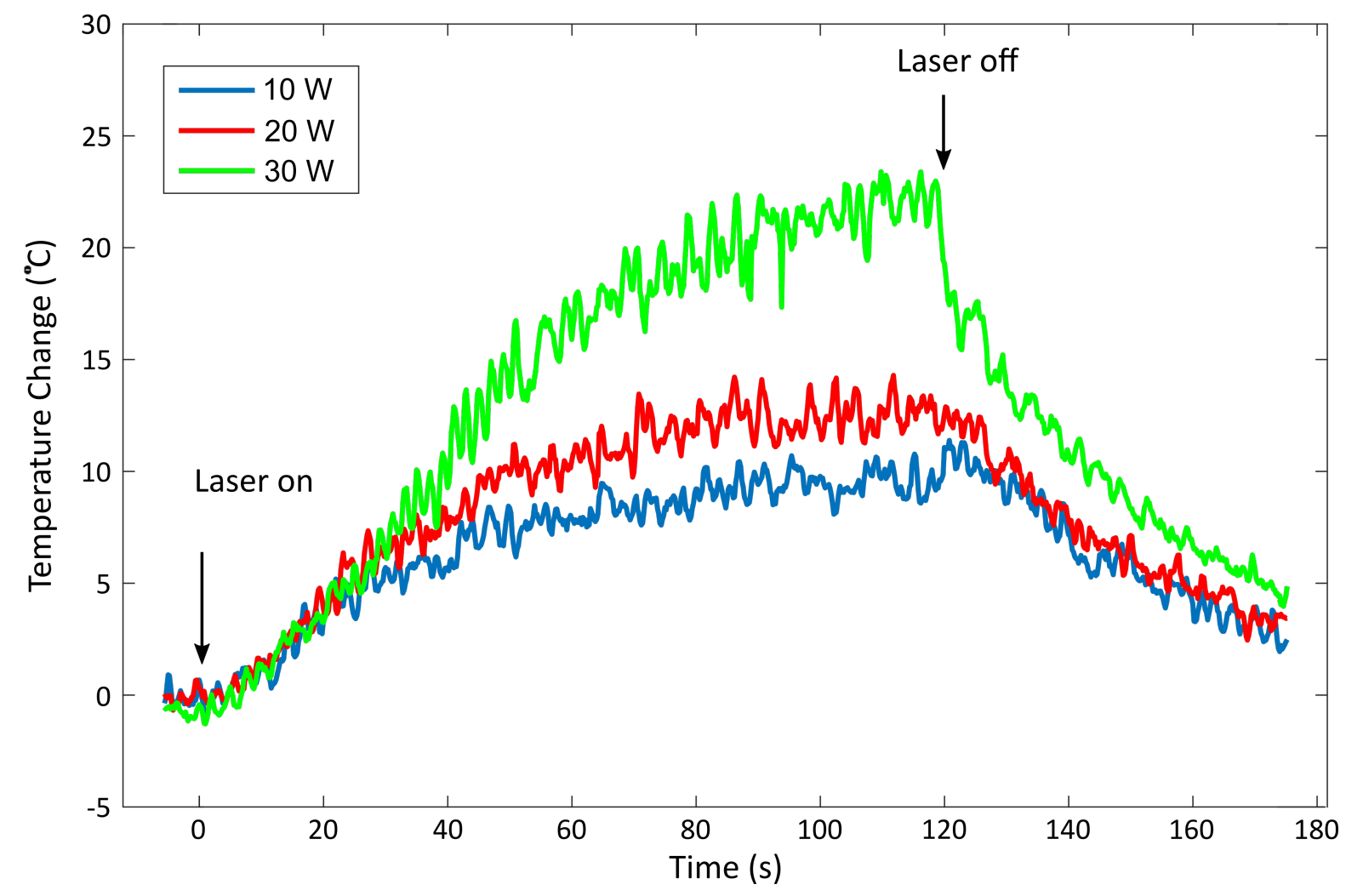

Figure 6.6.: Temperature changes of a ROI (depicted in Figures 6.2, 6.3 and 6.4) next to the tip of the laser fiber obtained before and during $120 \mathrm{~s}$ of laser pulsing with different output power and energy.

is pushed out of the selected imaging plane in the images during and after heating. Two reasons may contribute to the presence of the 'black hole'. Due to the thermal accumulation of the fiber tip, high temperature increases occur in the small region (about of $1 \mathrm{~mm}$ ), which cause the tissue ablation and generate steam bubbles. On the other hand, as observed in the MR images acquired after heating and the post-treatment photo, the diameter of the 'black hole' is almost the same as the diameter of the coagulated lesion (about $3 \mathrm{~mm}$ ). Therefore, the deproteinized tissue may also cause a signal loss in the proton density weighted image.

\subsubsection{Single Laser Pulse \& Temperature}

One major advantage of applying real-time MRI technique in temperature mapping is that it has the potential to observe fast temperature changes during a minimally invasive surgery. In the following experiment, fast temperature variations will be achieved by applying single pulsed laser energy in the kidney. 


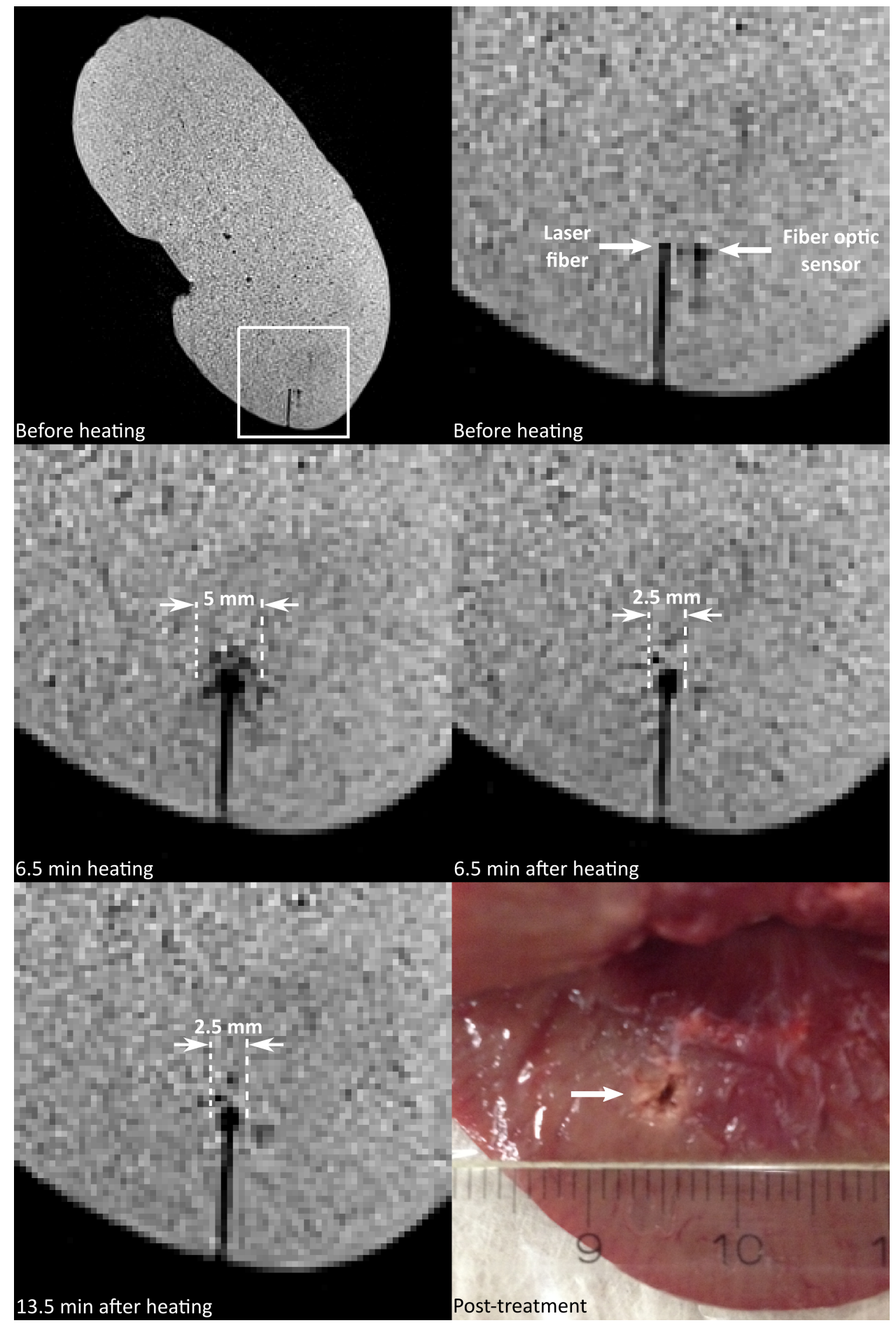

Figure 6.7.: Magnified proton density weighted images (voxel size: $0.5 \times 0.5 \times 0.5 \mathrm{~mm}^{2}$ ) obtained before and after laser pulsing (output power $=3 \mathrm{~W}$ and output energy $=234 \mathrm{~J}$, for other parameters see text). The lesion created by the laser is shown in the posttreatment photo. 


\section{Methods and Materials}

Three single laser pulses were applied with a $20 \mathrm{~s}$ break between each other. For each pulse, the output power was $30 \mathrm{~W}$ (maximum output) and the pulse duration was $200 \mathrm{~ms}$, which is the same as the temporal resolution of MR thermometry sequence (Table 6.1). The MR sequence was started a few seconds before the first laser pulse to acquired temperature reference data and stopped $40 \mathrm{~s}$ after the last laser pulse.

\section{Results}

Magnified temperature difference maps acquired after the use of the three pulses are shown in Figure 6.8(a). Due to the fact that the thermal diffusivity in the kidney cortex is a constant value, the diameter of the heated region increases with the increase of the pulse number to about $4 \mathrm{~mm}, 5 \mathrm{~mm}$ and $6 \mathrm{~mm}$, respectively. This finding is also confirmed in Figure 6.9. According to the location of a ROI (indicated in Figure 6.8 (a)), the temperature change during the experiment is shown in Figure 6.8 (b). Temperature increases immediately after each pulse with the maximum temperature increases of about 6.5, 9.5 and $12{ }^{\circ} \mathrm{C}$. As expected, a temperature increase of about $7.5^{\circ} \mathrm{C}$ can be observed after each pulse, because the same amount of energy $(6 \mathrm{~J})$ is introduced into the kidney.

\subsubsection{Laser Experiments in Kidney Medulla}

Previous experiments are performed in the kidney cortex, however tumor can also occur in the kidney medulla, which has a different biological structure with more large vessels. In the following experiment, laser heating is conducted in the kidney medulla, the temperature changes are monitored with real-time MR thermometry and the corresponding anatomical image and temperature maps are derived and analyzed.

\section{Methods and Materials}

The laser fiber and the temperature sensor were inserted $2 \mathrm{~cm}$ into the kidney medulla (as shown in Figure 6.10). In order to create a lesion, the following laser parameters were employed: pulsed mode, output power $=10 \mathrm{~W}$, repetition rate $=0.5 \mathrm{~Hz}$, pulse duration $=100 \mathrm{~ms}$, total applying time $=180 \mathrm{~s}$ and output energy $=90 \mathrm{~J}$. The MR thermometry sequence was started a few seconds before the laser was applied until the heating procedure was completed. $T_{1}$ weighted images were acquired before and after heating to collect anatomical information $\left(\mathrm{TE} / \mathrm{TR}=2.46 / 350 \mathrm{~ms}\right.$, flip angle $=70^{\circ}$, slice thickness $=3 \mathrm{~mm}$, in-plane resolution $=0.28 \times 0.28 \mathrm{~mm}^{2}, \mathrm{FOV}=180 \times 180 \mathrm{~mm}^{2}$, number of averages $=2$ ). 


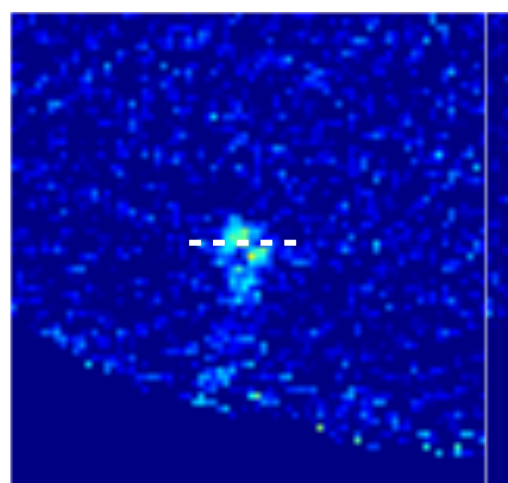

Pulse 1

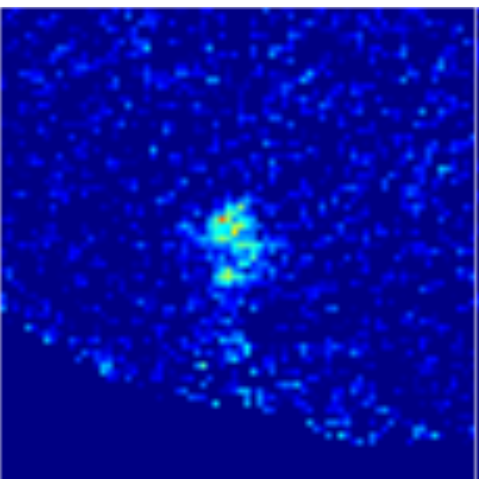

Pulse 2

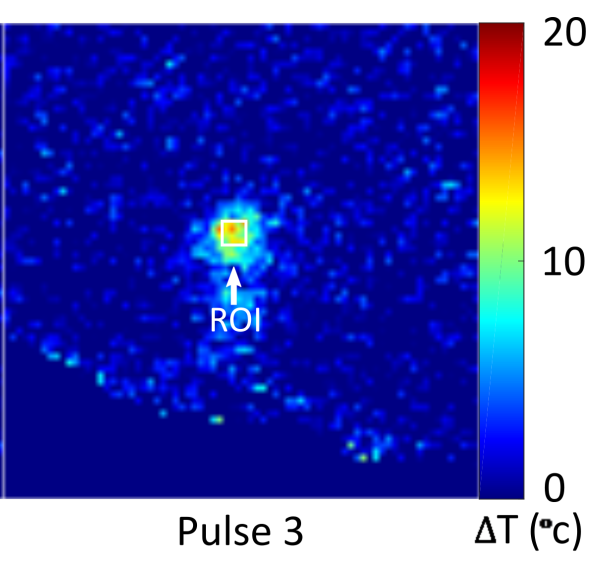

Pulse 3

(a)

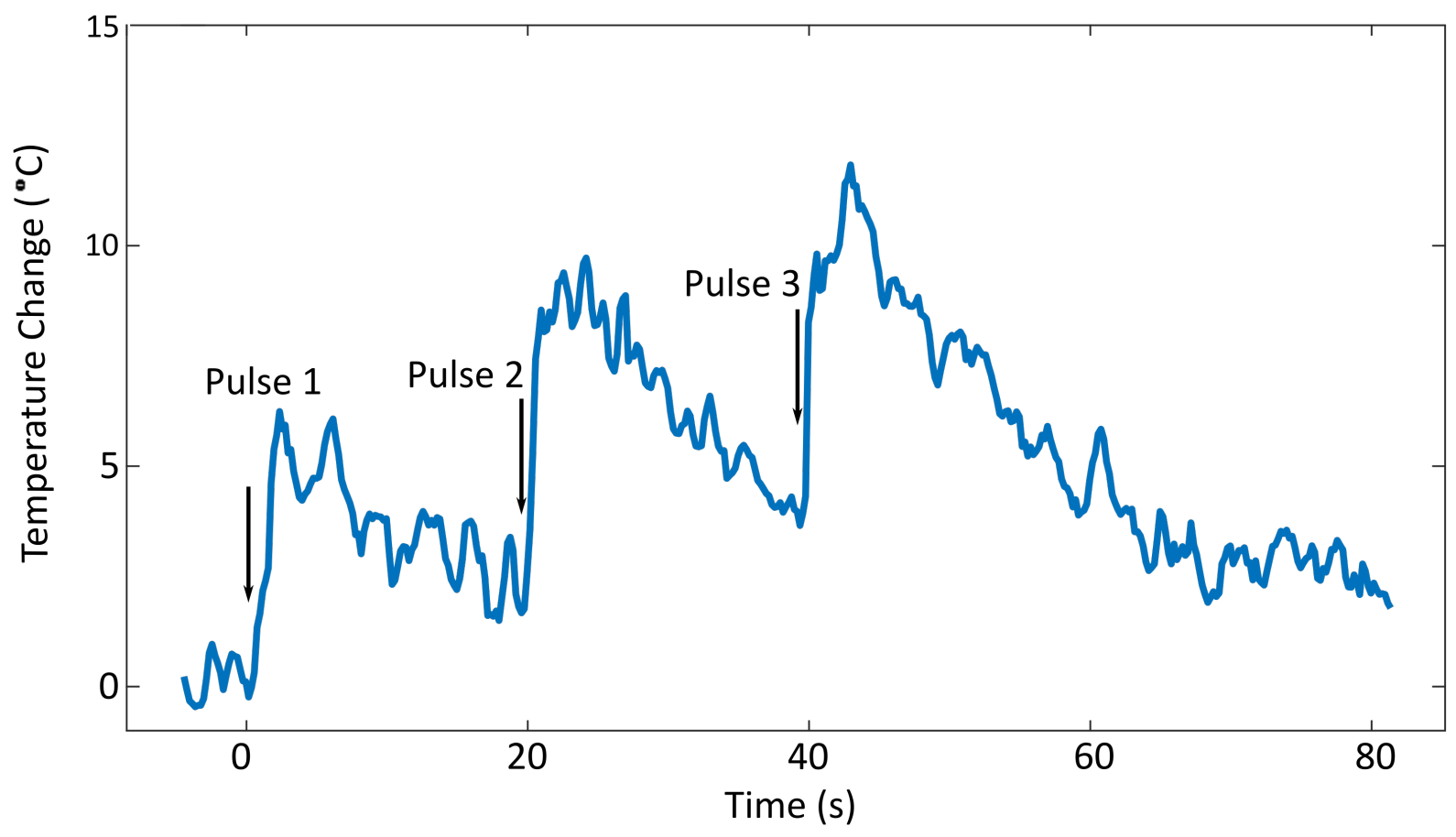

(b)

Figure 6.8.: (a) Magnified temperature difference maps obtained after a single laser pulse at 0,20 and $40 \mathrm{~s}$. The temperature profile along the dashed line of the three pulses is illustrated in Figure 6.9. (b) Temperature change of the ROI (shown in (a)) during the experiment. The applied laser parameters are: single pulse mode, output power $=30 \mathrm{~W}$, output energy $=6 \mathrm{~J}$ per pulse, pulse duration $=200 \mathrm{~ms}$. 


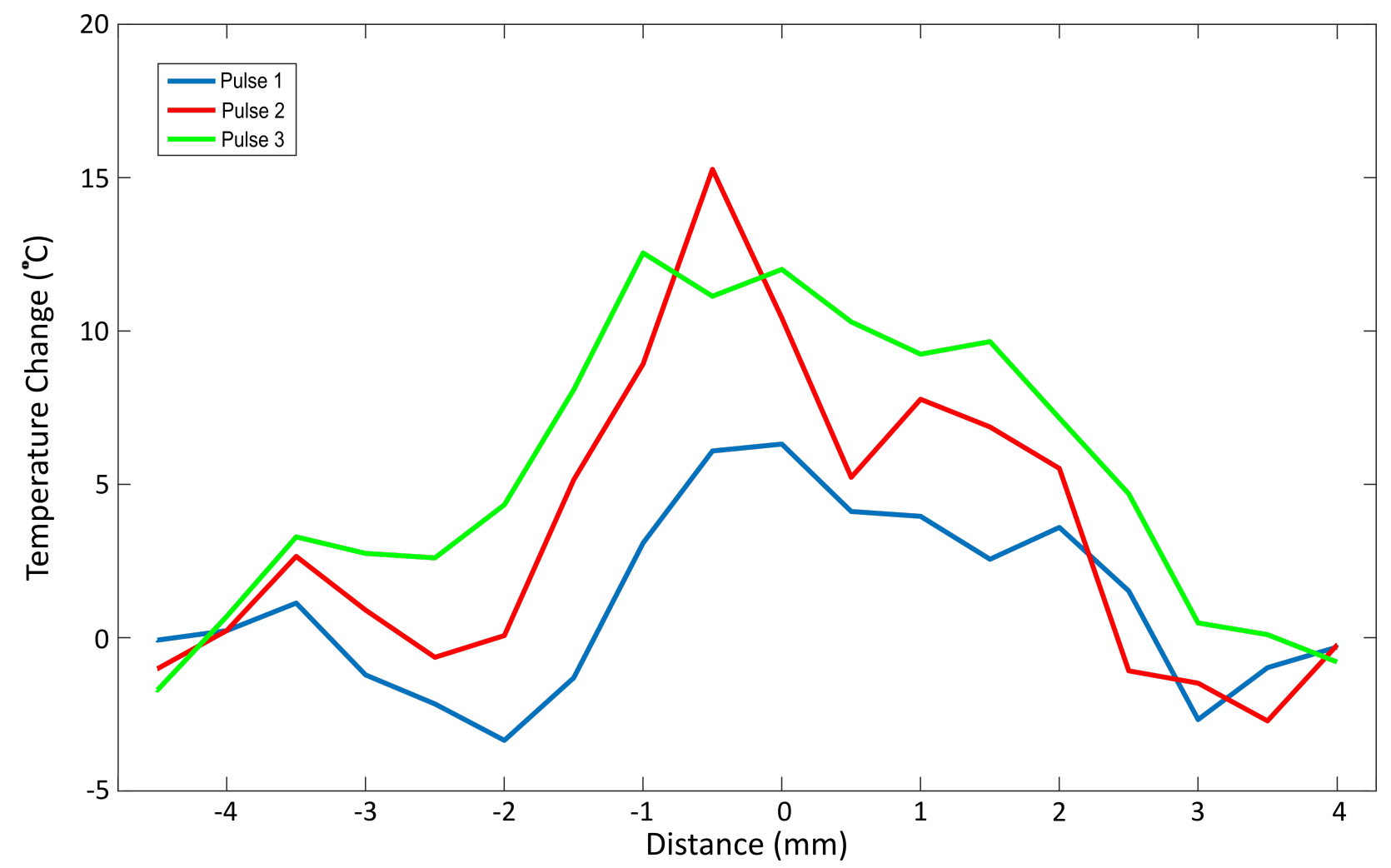

Figure 6.9.: Temperature profile along the dashed line (indicated in Figure 6.8) obtained after three single laser pulses.

\section{Results}

As shown in Figure 6.10, compared with the $T_{1}$ weighted image obtained before heating, a signal loss can be observed in the same location of the image acquired after the heating procedure. Similar to the previous results in Figure 6.2 (acquired under the same laser operational parameters), focal heating is realized by the energy deposition from the laser system (as shown in Figure 6.11). Different from the results acquired from the kidney cortex, as indicated by the arrows in Figure 6.11, temperature difference map acquired after $180 \mathrm{~s}$ of laser heating shows an asymmetric distribution around the fiber tip. In addition, signal drop can be observed distant to the tip of the laser fiber. One factor might contribute to this phenomenon that, the pressure of the vessel closed to the heating region is changes under the high temperature increase, which results in a movement of the concreted blood in the excised kidney. Therefore, a signal loss can be observed in the $T_{1}$ weighted image and the image acquired by the PRF thermometry sequence. This signal differences between the processed data and the reference generate the temperature artifacts in the temperature difference map (Figure 6.11). Moreover, the 'black hole' also appears in the kidney medulla in the anatomical images during heating. 


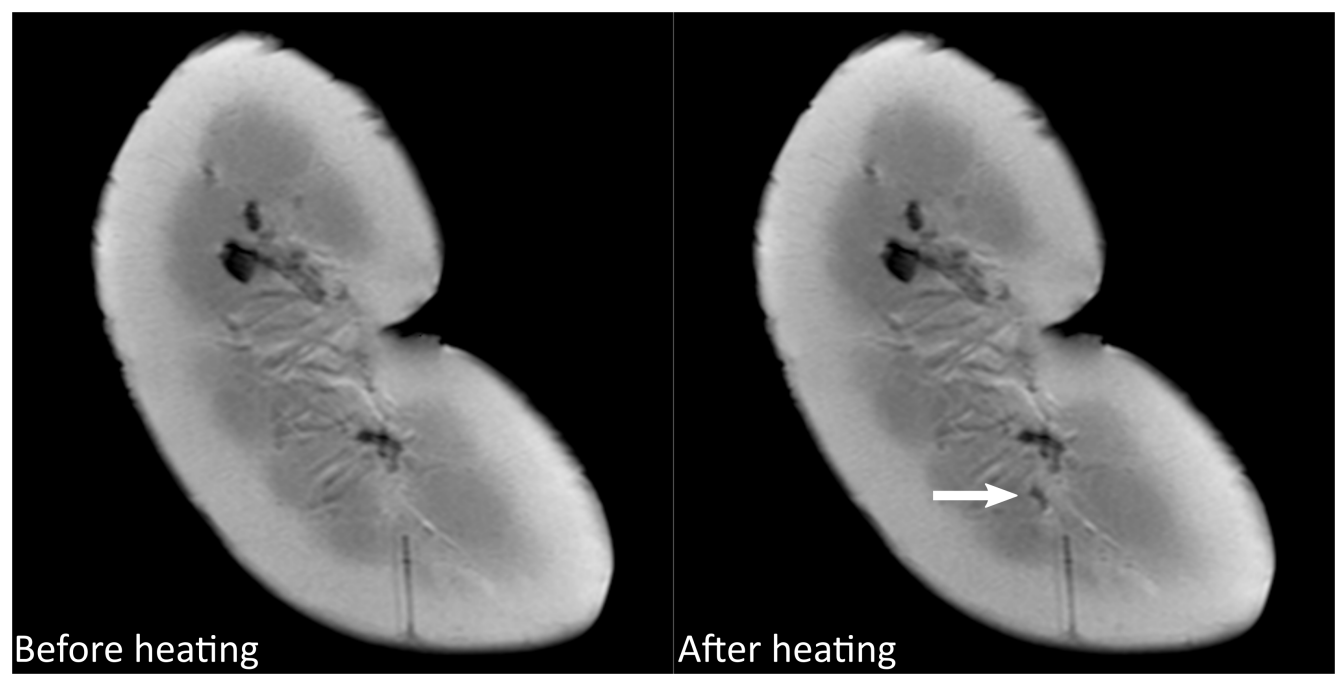

Figure 6.10.: Comparison between $T_{1}$ weighted images (voxel size: $0.3 \times 0.3 \times 3 \mathrm{~mm}^{2}$ ) acquired before and after laser pulsing (output power and energy are $10 \mathrm{~W}$ and $90 \mathrm{~J}$, respectively) in the kidney medulla. A signal loss distant to the tip of the laser fiber can be observed in the medulla (arrow).

\subsubsection{Laser Protocols under Minimally Invasive Surgery Conditions}

As introduced in Chapter 3, the tissue coagulation therapy in routine clinical practice is usually achieved by increasing the temperature of the target tissue from body temperature to $50-60{ }^{\circ} \mathrm{C}$ for several minutes. On the other hand, the wavelength of the laser system employed in present work is $2 \mu \mathrm{m}$, which consequently has a penetration depth of about $1 \mathrm{~mm}$ in the aqueous tissue. Therefore, proper laser parameters are applied in the following experiment to increase the temperature of the target tissue for more than $15{ }^{\circ} \mathrm{C}$ and meanwhile to avoid unexpected tissue damage by high laser output power.

\section{Methods and Materials}

The laser system was operated in the pulsed mode with the following parameters: output power $=2 \mathrm{~W}$, repetition rate $=20 \mathrm{~Hz}$, pulse duration $=20 \mathrm{~ms}$, total applying time $=180 \mathrm{~s}$ and output energy $=144 \mathrm{~J}$. The MR thermometry sequence was started a few seconds before the laser heating until the laser was turned off for $1 \mathrm{~min}$. Temperature maps as well as two magnitude images at different TE were calculated.

\section{Results}

With PRF based real-time MR thermometry, two anatomical images with different contrasts as well as the temperature maps are provided. As demonstrated in the temperature map, the focal heating lesion is realized by laser energy deposition, the maximum temperature rise in the central region is around $20^{\circ} \mathrm{C}$. Additionally, Figure 6.12 (b) illustrates the temperature change of a ROI ( $2 \mathrm{~mm}$ from the tip of the laser fiber) during and after 


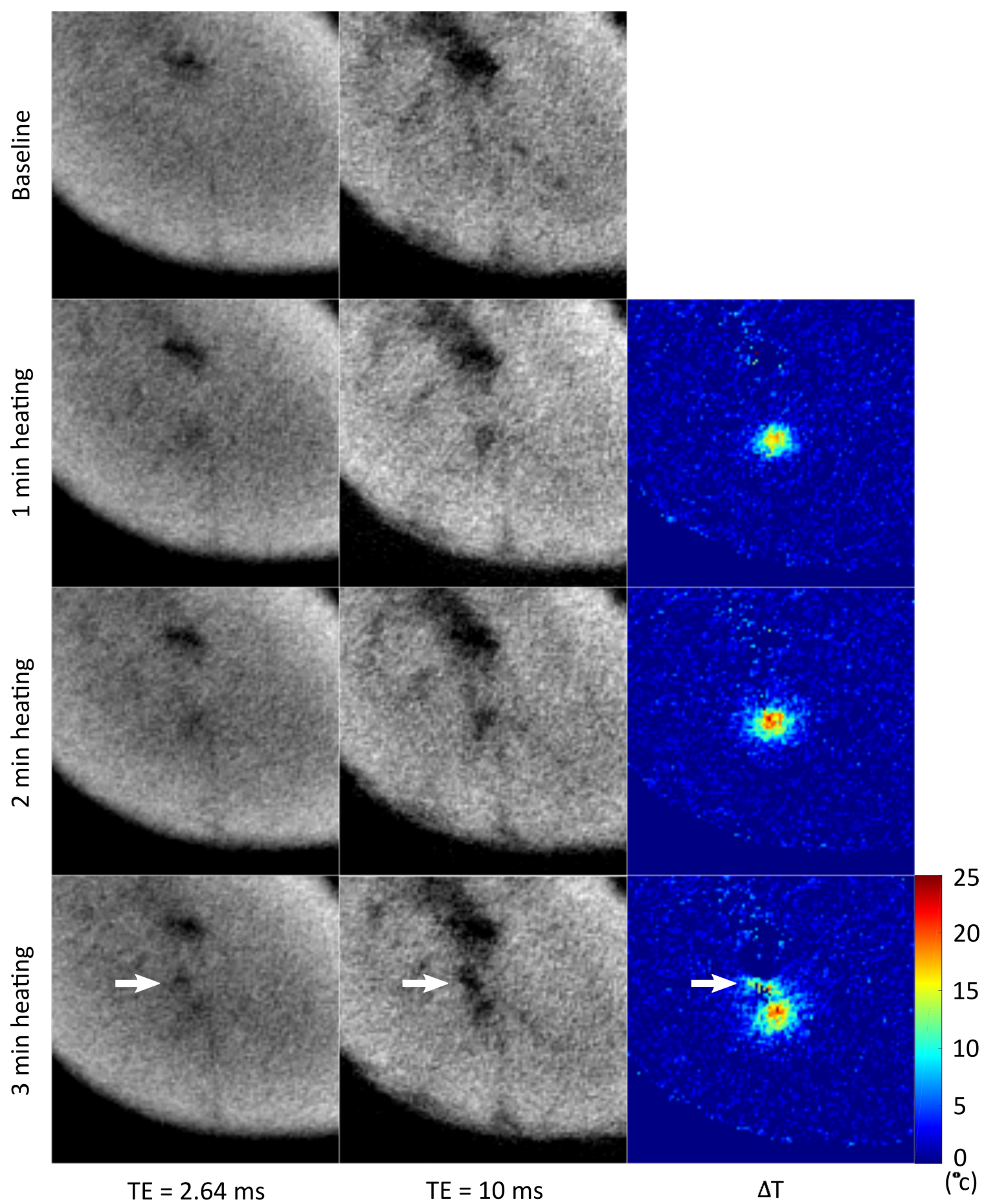

Figure 6.11.: Magnified MR magnitude images of a kidney before and during $180 \mathrm{~s}$ of laser pulsing in the medulla (output power $=10 \mathrm{~W}$, output energy $=90 \mathrm{~J}$, repetition rate $=0.5 \mathrm{~Hz}$, pulse duration $=100 \mathrm{~ms}$ ) obtained with a $\mathrm{TE}=2.64 \mathrm{~ms}$ (left column) and TE $=10 \mathrm{~ms}$ (middle column). Magnified relative temperature difference maps (right column) were calculated from the corresponding images obtained with $\mathrm{TE}=10 \mathrm{~ms}$. After $180 \mathrm{~s}$ of laser pulsing, a signal loss above the tip of the laser fiber is observed in the magnitude images as well as in the temperature maps (arrows). 
the heating procedure. The temperature is raised by $15{ }^{\circ} \mathrm{C}$ rapidly during the first $40 \mathrm{~s}$ of heating and reaches a plateau of about $18{ }^{\circ} \mathrm{C}$ after $100 \mathrm{~s}$ of heating. After switching off the laser system, the temperature drops dramatically and is decreased by almost $15{ }^{\circ} \mathrm{C}$ after $60 \mathrm{~s}$. Moreover, the 'black hole' can still be observed in the central heating area, even though the laser parameters are optimized for the experiment.

\subsection{Summary}

In order to better mimic minimally invasive surgery procedures, a laser system is introduced to generate a focal lesion. Corresponding temperature changes are successfully monitored by the PRF based real-time MR thermometry. A spatial resolution of $0.5 \times 0.5 \times 3 \mathrm{~mm}^{3}$ and a temporal resolution of $200 \mathrm{~ms}$ per frame are achieved for data acquisition. 'Black holes' appear during the heating procedure, which might be due to the tissue ablation and deproteination close to the tip of the laser fiber. Further more, experiments in kidney medulla shows unexpected temperature changes, because of the presence of large blood vessels, which in return proves that it is necessary to perform temperature mapping during the thermal therapy. Lastly, in the experiment with optimized laser protocols, real-time MR thermometry indicates that the temperature can be raised by $15^{\circ} \mathrm{C}$ after $40 \mathrm{~s}$ laser heating. A increase of $20^{\circ} \mathrm{C}$ within $180 \mathrm{~s}$ was achieved. 


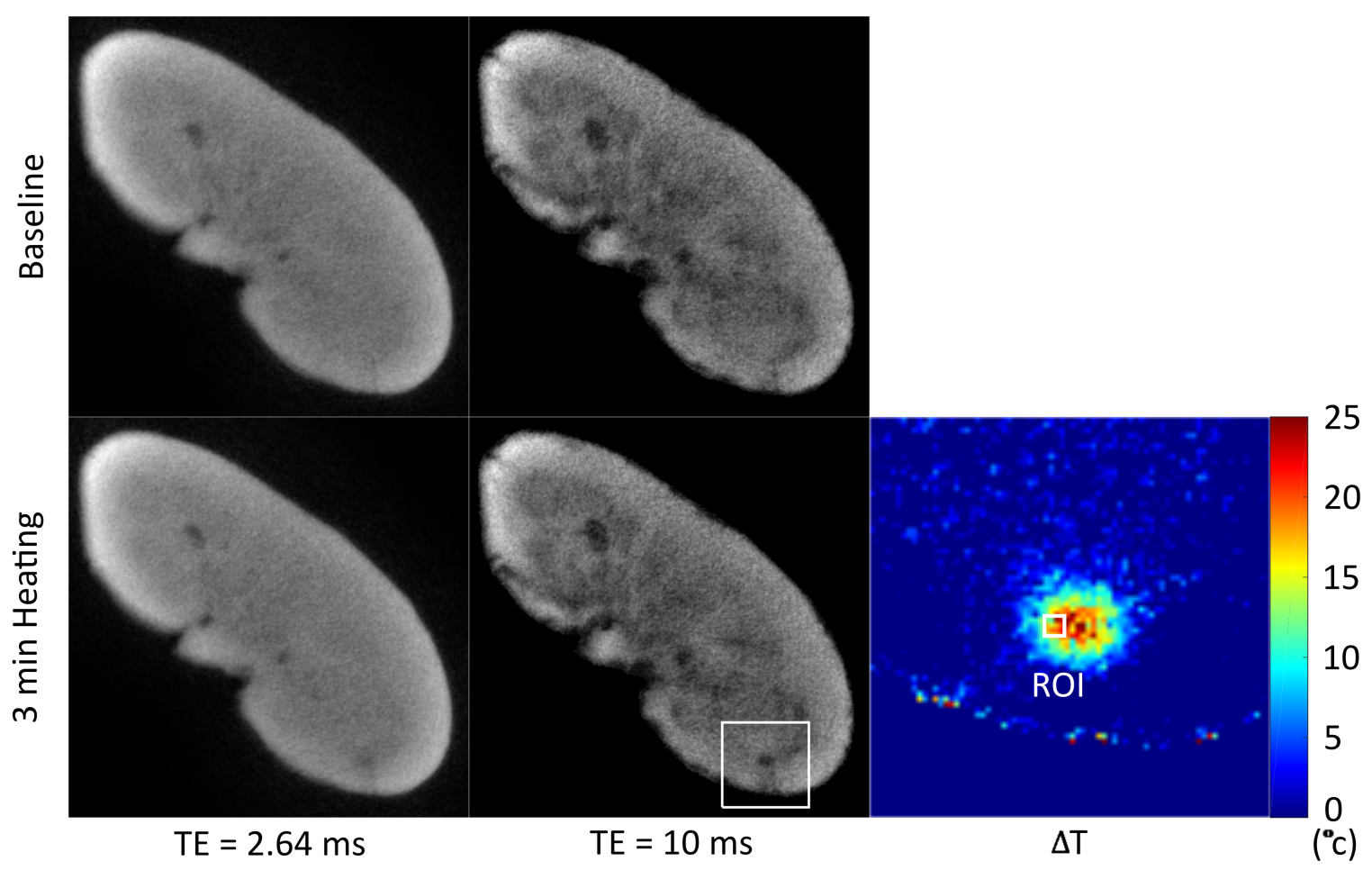

(a)

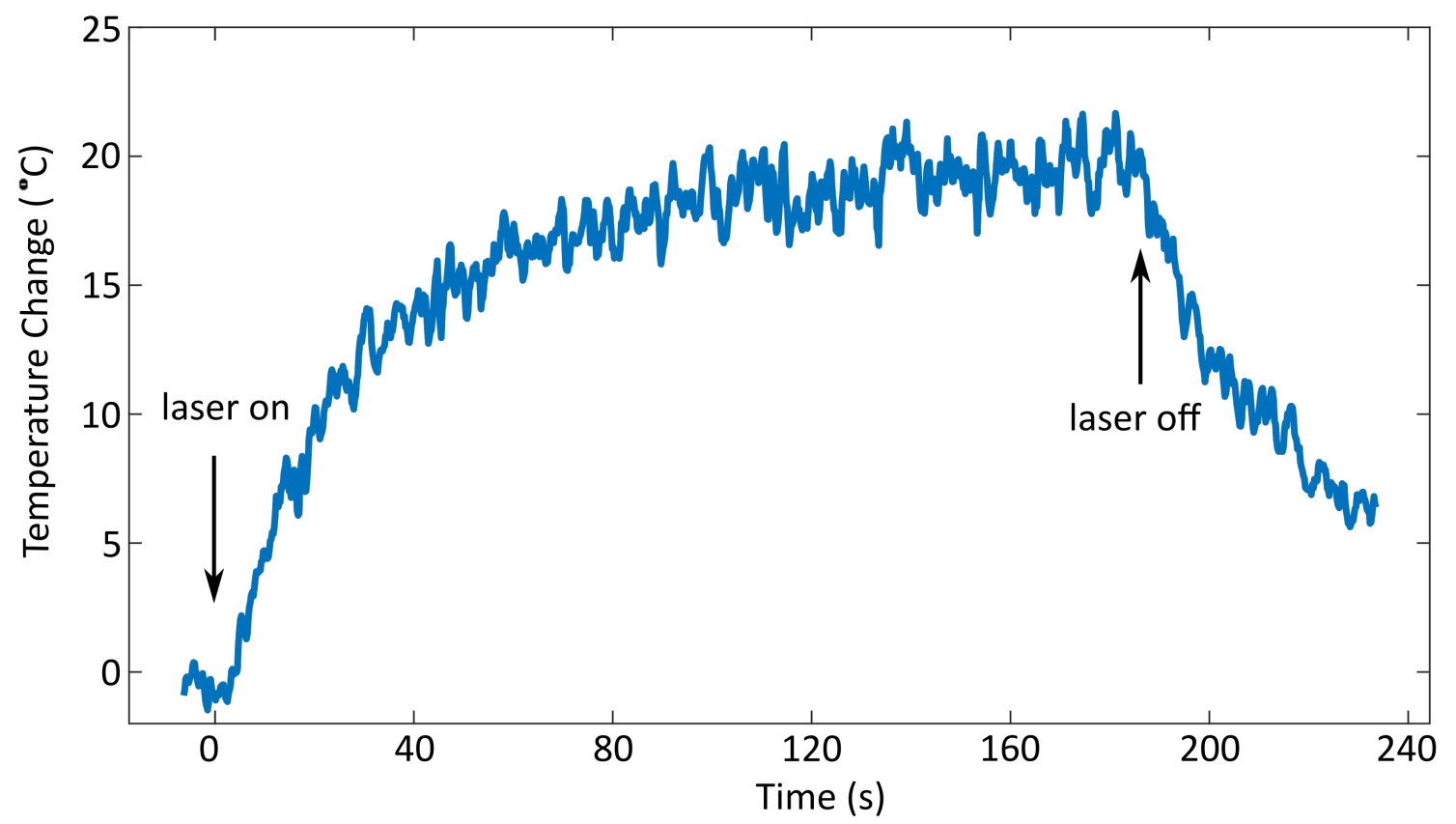

(b)

Figure 6.12.: (a) MR magnitude images of a kidney before and during $180 \mathrm{~s}$ of laser pulsing in the cortex (output power $=3 \mathrm{~W}$, output energy $=143 \mathrm{~J}$, repetition rate $=$ $10 \mathrm{~Hz}$, pulse duration $=20 \mathrm{~ms}$ ) obtained with a $\mathrm{TE}=2.64 \mathrm{~ms}$ (left column) and $\mathrm{TE}$ $=10 \mathrm{~ms}$ (middle column). Magnified (as indicated by the box) temperature difference map (right) were calculated from the corresponding image obtained with $\mathrm{TE}=10 \mathrm{~ms}$.

(b) The temperature change of a ROI next to the tip of the laser fiber. 



\section{Reference Choice under In Vivo Conditions}

\subsection{Introduction}

Motion induced artifacts in MR thermometry are generally divided into two categories. The first kind is the intra-scan motion, which results from the tissue movement during the data acquisition of a single frame. Blurring artifacts in anatomical as well as temperature images are often observed with the low temporal resolution sequences. In the present work, this problem is solved by applying real-time MRI technique. As demonstrated in previous chapters, a temporal resolution of $200 \mathrm{~ms}$ per fame for data acquisition is used in the experiments and is sufficient to eliminate artifacts caused by intra-scan motion, e.g. respiration [77]. The second kind is the inter-scan motion. In order to derive temperature differences during the coagulation procedure, PRF based MR thermometry requires the data sampled before heating as a reference. The tissue displacement between the current processed and the reference data sets leads to local magnetic field variations and susceptibility effects, which cause additional phase shifts and contribute to temperature calculation errors. Many approaches have been proposed to solve problems induced by inter-scan motion.

Motion restraining techniques, such as 'respiratory gating' and navigator echoes, are employed to minimize periodic motion artifacts by synchronizing the data acquisition to a certain phase of the periodic cycle [77, 106-108]. The major disadvantage of these techniques is that the temperature updating time depends on the duration of the periodic motion. For instance, due to respiratory motion, the corresponding temporal resolution in humans would be about $5 \mathrm{~s}$. Therefore, motion restraining techniques are not suitable for real-time MR thermometry.

Another approach, referenceless (also called self-reference) thermometry, is also used in MR temperature mapping to remove inter-scan motion artifacts. Instead of acquiring the reference data, this method estimates the reference information from the unheated area of every individual frame during the heating procedure. Subsequently, the reference phase value is calculated from a polynomial function, the corresponding polynomial coefficients are determined from the chosen unheated region by applying a least-squares fit [109, 110]. 
However, one precondition of performing referenceless thermometry is that the SNR in the chosen region needs to be sufficient for the background estimation. This requirement can not always be fulfilled in the present work. Firstly, the inserted laser fiber disturbs the local magnetic field that raises new problems during the polynomial fitting procedure. In addition, PRF based real-time MR thermometry employs all the coil sensitivity maps derived from the NLINV reconstruction for the phase difference calculation. More distant coils have a low sensitivity in the region of the focal lesion, which leads to an over-estimation or an under-estimation of the preheating background.

In this chapter, a reference selection procedure is applied in real-time MR thermometry to reduce the inter-scan motion artifacts. The method combines multi-baseline acquisition and image similarity test and its stability is assessed under the in vivo condition.

\subsection{Methods and Materials}

\section{MR Thermometry}

A healthy volunteer was recruited for the experiment and a 16-channel body coil was employed for data acquisition. In order to image the abdomen region of the volunteer and keep the temporal resolution, the FOV and the spatial resolution are set to $256 \times 256 \mathrm{~mm}^{2}$ and $0.5 \times 0.5 \times 5 \mathrm{~mm}^{3}$, respectively. More MR parameters are summarized in Table 7.1. The experiment was performed for $100 \mathrm{~s}$ without thermal deposition. The acquired data from the first $90 \mathrm{~s}$, which covers several respiratory cycles, was considered as the multibaseline library, while the data from the last $10 \mathrm{~s}$ were used to calculate temperature difference maps.

Table 7.1.: MR parameters for PRF thermometry in volunteer studies.

\begin{tabular}{cc|cc}
\hline Sequence & Radial FLASH & FOV & $256 \times 256 \mathrm{~mm}^{2}$ \\
\hline $\mathrm{TE}_{1} / \mathrm{TE}_{2} / \mathrm{TR}$ & $2.64 / 10 / 11.9 \mathrm{~ms}$ & In-plane resolution & $0.5 \times 0.5 \mathrm{~mm}^{2}$ \\
\hline Flip angle & $10^{\circ}$ & Number of spokes & 17 per frame \\
\hline Slice thickness & $5 \mathrm{~mm}$ & Temporal resolution & $200 \mathrm{~ms}$ \\
\hline
\end{tabular}

\section{Data Post-processing}

Figure 7.1 gives an overview of the proposed reference selection scheme that combines the multi-baseline and the image similarity approaches [111-113]. In order to identify the cor- 
rect reference for temperature mapping, the image similarities were calculated between the current processed magnitude image and all the magnitude images in the reference library and the data with the highest similarity served as the reference data. The image similarity calculation was achieved by performing the cross-correlation algorithm, which is expressed as:

$$
C=\frac{\sum_{n=1}^{x}\left(A_{n}-\bar{A}\right)\left(B_{n}-\bar{B}\right)}{\sqrt{\left(\sum_{n=1}^{x}\left(A_{n}-\bar{A}\right)^{2}\right)\left(\sum_{n=1}^{x}\left(B_{n}-\bar{B}\right)^{2}\right)}}
$$

where $C$ is the cross-correlation coefficient, $A$ and $B$ are the chosen images and the corresponding mean values of the signal intensities are $\bar{A}$ and $\bar{B}, n$ represents the pixel index in the image ( $x$ pixels in total). Once the reference data is determined, the relative temperature map is calculated based on Equation (3.9).

\subsection{Results}

Due to the fact that there is no thermal energy deposited during the experiment, the computed temperature differences are expected to be close to $0{ }^{\circ} \mathrm{C}$. The lack of signal within the lung leads to a low signal intensity in the corresponding area, which is not of interest in the present work. Figure 7.2 (a) shows an example of a temperature difference map calculated with an arbitrary reference image. According to the dashed lines, the two anatomical images belong to different respiratory phases. One of the signal intensity differences is demonstrated by the arrows. The tissue displacement between the magnitude images results into apparent temperature changes with absolute values of more than $20{ }^{\circ} \mathrm{C}$ (light red: $20{ }^{\circ} \mathrm{C}$, light blue: $-20{ }^{\circ} \mathrm{C}$ ). In contrast, Figure $7.2(\mathrm{~b})$ shows the results calculated from the proposed reference selection procedure. As indicated by the dashed lines and the arrows, the two magnitude images are almost visually identical. Additionally, the inter-scan motion induced artifacts in area of the abdomen are dramatically minimized by applying the multi-baseline and image similarity methods. According to the locations of the two kidneys in the volunteer, two ROIs are selected for quantitatively assessing the stability of the proposed method (Figure 7.3). As expected, all the obtained mean values are around $0{ }^{\circ} \mathrm{C}$ and the corresponding SDs are lower than $2.5^{\circ} \mathrm{C}$. 


\subsection{Summary}

In this chapter, multi-baseline and image similarity methods are successfully adopted to PRF based real-time MR thermometry in vivo to eliminate periodic motion induced temperature errors without breath holding or external motion registration techniques (e.g. respiratory gating). In the case of no thermal deposition, the calculated temperature changes are close to $0{ }^{\circ} \mathrm{C}$. Due to the fact that real-time MRI employs the sliding window technique for data acquisition [1], it is necessary to use a library, which contains several breathing cycles, so that the anatomical images of the same sampling pattern cover at least one cycle for the image similarity test. In conclusion, with the proposed method, MR thermometry in the human abdomen might be feasible during thermal therapy without sacrificing the temperature updating time. 


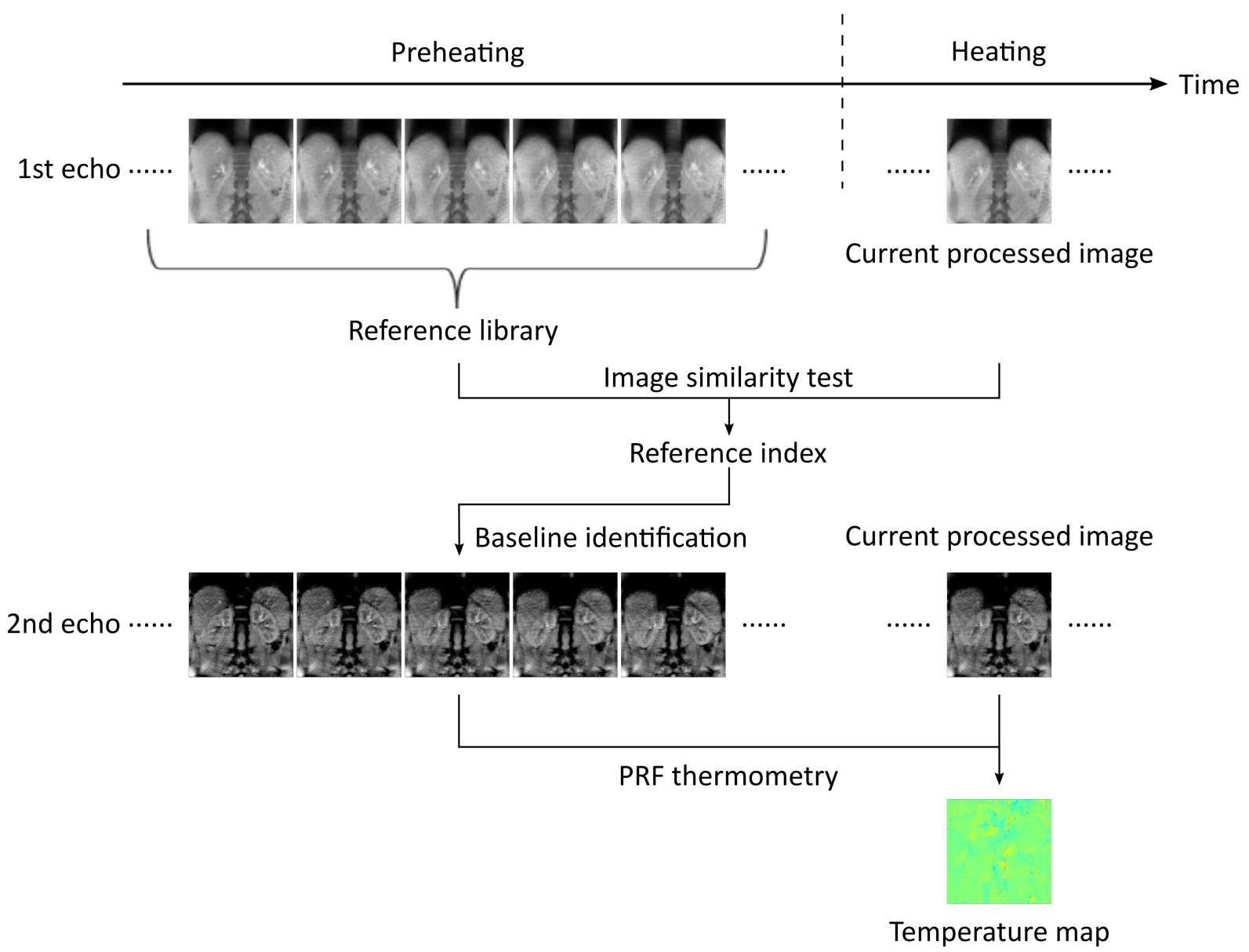

Figure 7.1.: The reference selection procedure for PRF based real-time MR thermometry. The magnitude images reconstructed from the first echo acquired before heating are employed as the reference library. The image similarity is computed between the current processed data during heating (from the first echo) and every individual image in the reference library. Once the data set with the highest similarity is located, the corresponding image of the second echo is used as the baseline for temperature calculation. 


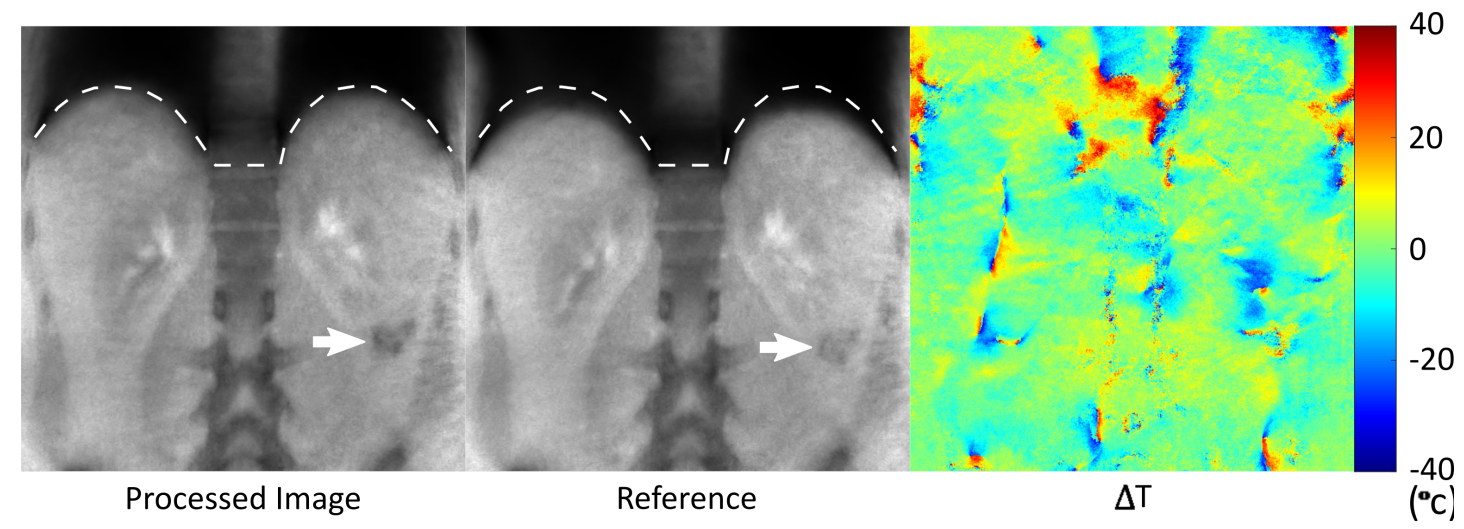

(a)

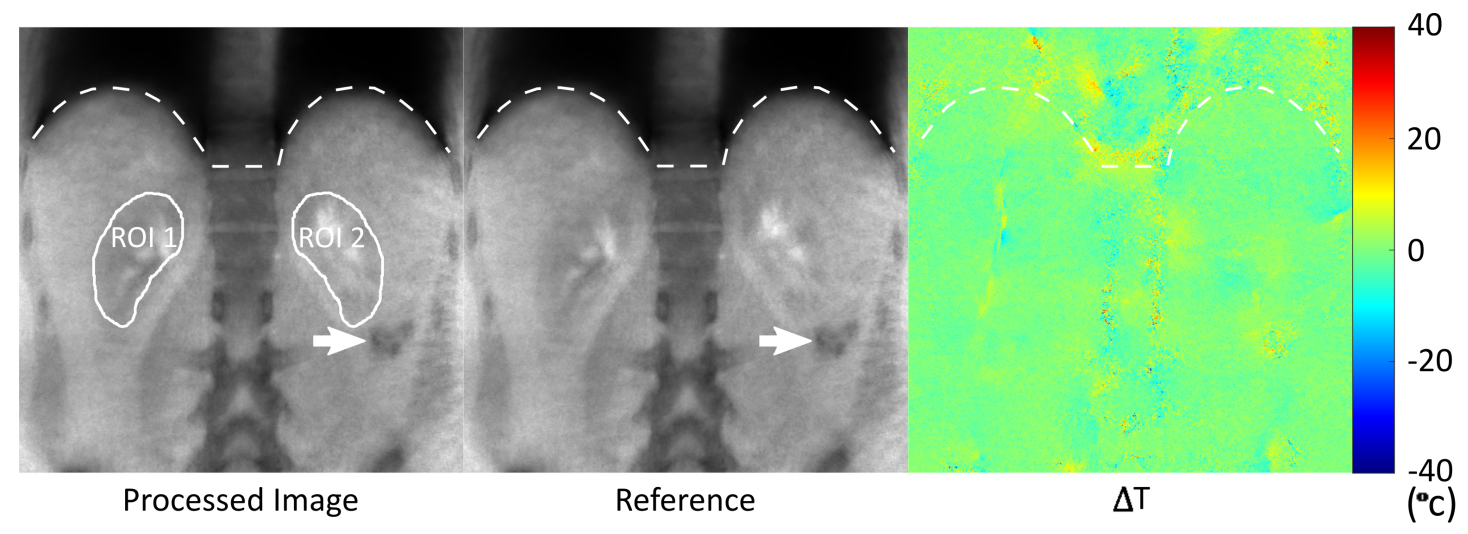

(b)

Figure 7.2.: (a) The temperature difference map (right) calculated from a magnitude image (left) and a randomly selected reference image (middle) of a human abdomen. (b) The temperature difference map (right) calculated from the same image (left) and a reference chosen by the multi-baseline and the image similarity approaches (middle). Temperature values of the two ROIs of the kidney are employed for the evaluation of the proposed method in Figure 7.3. 


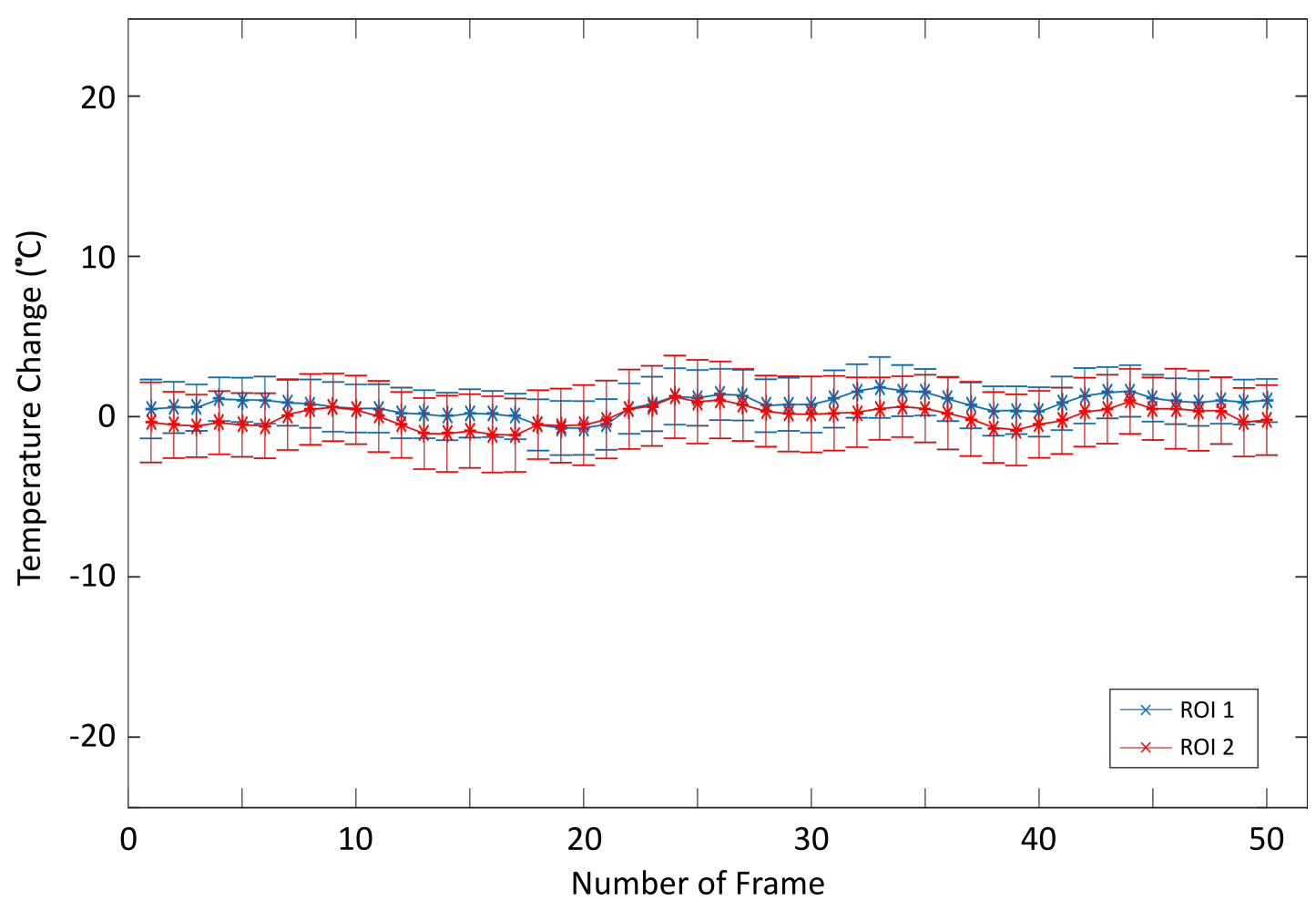

Figure 7.3.: Calculated temperature changes (mean \pm SD) of two ROIs (human kidney) from 50 successive frames. ROIs are depicted in Figure 7.2 (b). 



\section{Model-based Reconstruction for MR Thermometry}

\subsection{Introduction}

In previous chapters, in order to calculate the temperature changes, complex images of the current processed and the reference datasets are reconstructed separately and followed by a multi-channel phase contrast calculation. Meanwhile, as discussed in Chapter 6, in order to localize the laser fiber during the heating procedure, the MR sequence with high spatial resolution $\left(0.5 \times 0.5 \times 3 \mathrm{~mm}^{3}\right)$ is employed. The limited voxel size leads to a SNR decrease in the magnitude images as well as the PRF shift induced temperature maps. Therefore, in this chapter, a model-based reconstruction strategy, which jointly calculates the complex anatomical images and the temperature difference maps, is proposed to improve the SNR of the related parameter maps. Similar to NLINV, the model-based reconstruction for MR thermometry is also achieved by applying the iteratively regularized Gauss Newton method.

\subsection{Theory}

\subsubsection{PRF MR Thermometry as Nonlinear Inverse Problem}

As introduced in Chapter 6, in the present work, MR signals acquired at difference TE $(2.64 \mathrm{~ms}$ and $10 \mathrm{~ms})$ are used to provide anatomical images with two different contrasts. In the proposed model-based reconstruction, the images acquired with the short TE and the corresponding coil sensitivity maps $\left(c_{j}\right)$ are computed by the routine NLINV algorithm. In addition, during thermal therapies, the raw data of the temperature baseline is located according to the reference selection procedure mentioned in Chapter 7. As a consequence, the current processed and the chosen reference data with a TE of $10 \mathrm{~ms}$ can be assumed to share the same anatomical content $(\rho)$ and the corresponding MR signal of the second echo can be formulated as:

$$
\mathrm{y}_{j, l}(t)=\int \rho(\overrightarrow{\mathrm{x}}) \cdot e^{i \Delta \phi(\mathrm{x}) \cdot l} \cdot c_{j}(\overrightarrow{\mathrm{x}}) \cdot e^{-i 2 \pi \vec{k}_{l}(t) \overrightarrow{\mathrm{x}}} d \overrightarrow{\mathrm{x}} \quad \text { with } j \in[1, N], l \in[0,1]
$$


where $l=0$ and $l=1$ represent the reference and the current processed data, respectively, $\Delta \phi$ is the temperature induced phase difference between the two datasets and $\vec{k}(t)$ denotes the k-space trajectory.

Similar to $[1,36,114]$, with $\rho$ and $\Delta \phi$ being unknowns, Equation (8.1) represents a nonlinear operator, which can be simplified as:

$$
F(\mathrm{x})=\mathrm{y} \text { with } x=\left(\begin{array}{c}
\rho \\
\Delta \phi
\end{array}\right)
$$

with y denoting the acquired raw data and $\mathrm{x}$ containing the unknown parameters. The forward operator $F$ transfers the unknowns and the input coil profiles to k-space:

$$
F: x \mapsto\left(\begin{array}{c}
P_{0} \mathcal{F}\left\{\rho \cdot e^{i \Delta \phi \cdot 0} \cdot c_{1}\right\} \\
\vdots \\
P_{0} \mathcal{F}\left\{\rho \cdot e^{i \Delta \phi \cdot 0} \cdot c_{N}\right\} \\
P_{1} \mathcal{F}\left\{\rho \cdot e^{i \Delta \phi \cdot 1} \cdot c_{1}\right\} \\
\vdots \\
P_{1} \mathcal{F}\left\{\rho \cdot e^{i \Delta \phi \cdot 1} \cdot c_{N}\right\}
\end{array}\right)
$$

where $\mathcal{F}$ is FFT and $P_{l}$ projects the data onto the $l_{t h}$ trajectory. To solve this nonlinear inverse problem, Gauss Newton method is applied to linearize Equation (8.2), which leads the corresponding cost function to:

$$
\Phi(d \mathrm{x})=\left\|D F\left(\mathrm{x}_{n}\right) d \mathrm{x}-\left(\mathrm{y}-F\left(\mathrm{x}_{n}\right)\right)\right\|_{2}^{2}
$$

with $\mathrm{x}_{n}$ being the estimate from the $n^{\text {th }}$ Newton step and $D F(\mathrm{x})$ being the Fréchet derivative of $F$ at $\mathrm{x}_{n}$. According to [35, 36, 114], $D F(\mathrm{x})$ is derived by applying the linear property of the Fourier transform and the Jacobian matrix to $F$ :

$$
D F(\mathrm{x})\left(\begin{array}{c}
d \rho \\
d \Delta \phi
\end{array}\right)=\left(\begin{array}{c}
P_{0} \cdot \mathcal{F}\left\{e^{i \Delta \phi \cdot 0}\left(c_{1} \cdot d \rho+0 \cdot \rho \cdot c_{1} \cdot d \Delta \phi\right)\right\} \\
\vdots \\
P_{0} \cdot \mathcal{F}\left\{e^{i \Delta \phi \cdot 0}\left(c_{N} \cdot d \rho+0 \cdot \rho \cdot c_{N} \cdot d \Delta \phi\right)\right\} \\
P_{1} \cdot \mathcal{F}\left\{e^{i \Delta \phi \cdot 1}\left(c_{1} \cdot d \rho+1 \cdot \rho \cdot c_{1} \cdot d \Delta \phi\right)\right\} \\
\vdots \\
P_{1} \cdot \mathcal{F}\left\{e^{i \Delta \phi \cdot 1}\left(c_{N} \cdot d \rho+1 \cdot \rho \cdot c_{N} \cdot d \Delta \phi\right)\right\}
\end{array}\right)
$$

Furthermore, due to the fact that both the anatomical and the temperature images can be considered similar to the results of the previous frame during the heating procedure, 
Tikhonov regularization is employed in the cost function, which is rewritten as:

$$
\Phi(d \mathrm{x})=\left\|D F\left(\mathrm{x}_{n}\right) d \mathrm{x}-\left(\mathrm{y}-F\left(\mathrm{x}_{n}\right)\right)\right\|_{2}^{2}+\alpha_{n}\left\|\rho_{n}+d \rho-p \cdot \rho_{0}\right\|_{2}^{2}+\beta_{n}\left\|\Delta \phi_{n}+d \Delta \phi-q \cdot \Delta \phi_{0}\right\|_{2}^{2}
$$

where $\alpha_{n}$ and $\beta_{n}$ are the regularization parameters, and $p$ and $q$ are the damping factors on the initial guesses of the unknowns $\left(\rho_{0}\right.$ and $\left.\Delta \phi_{0}\right)$, respectively. The updating rule of Equation (8.6) is:

$$
\begin{aligned}
d \mathrm{x} & =d \rho+d \Delta \phi, \text { with } \\
d \rho & =\left[D F\left(\mathrm{x}_{n}\right)^{H} D F\left(\mathrm{x}_{n}\right)+\alpha_{n} I\right]^{-1}\left[D F\left(\mathrm{x}_{n}\right)^{H}\left(\mathrm{y}-F\left(\mathrm{x}_{n}\right)\right)+\alpha_{n}\left(\rho_{0}-\rho_{n}\right)\right] \\
d \Delta \phi & =\left[D F\left(\mathrm{x}_{n}\right)^{H} D F\left(\mathrm{x}_{n}\right)+\beta_{n} I\right]^{-1}\left[D F\left(\mathrm{x}_{n}\right)^{H}\left(\mathrm{y}-F\left(\mathrm{x}_{n}\right)\right)+\beta_{n}\left(\Delta \phi_{0}-\Delta \phi_{n}\right)\right]
\end{aligned}
$$

where $D F(\mathrm{x})^{H}$ is the adjoint of the Fréchet derivative and can be calculated by using the unitarity of $\mathcal{F}$,

$$
D F^{H}(\mathrm{x})\left(\begin{array}{c}
d \mathrm{y}_{1,0} \\
\vdots \\
d \mathrm{y}_{N, 0} \\
d \mathrm{y}_{1,1} \\
\vdots \\
d \mathrm{y}_{N, 1}
\end{array}\right)=\left(\begin{array}{c}
\sum_{j=1}^{N} c_{j}^{*} \cdot\left[\sum_{l=0}^{1}\left(e^{i \Delta \phi \cdot l}\right)^{*} \cdot \mathcal{F}^{-1}\left(P_{l}^{H} d \mathrm{y}_{j, l}\right)\right] \\
\sum_{j=1}^{N} c_{j}^{*} \cdot\left[\sum_{l=0}^{1}\left(l \cdot e^{i \Delta \phi \cdot l}\right)^{*} \cdot \rho^{*} \cdot \mathcal{F}^{-1}\left(P_{l}^{H} d \mathrm{y}_{j, l}\right)\right]
\end{array}\right)
$$

where $*$ represents the point-wise complex conjugation. Additionally, in the model-based reconstruction, in order to achieve the desired results within a reasonable number of Newton steps, a scaling factor $(s)$ is introduced in Equation (8.1) to ensure the balance among the products of all the partial derivatives. For the algorithm implementation, the data index $l$ is substituted by $\hat{l}=l \cdot s$ and $s$ is computed as [114]:

$$
s=0.5 \cdot\left(\left\|\mathrm{y}_{0}\right\|_{2}+\left\|\mathrm{y}_{1}\right\|_{2}\right) /\left\|\mathrm{y}_{0}-\mathrm{y}_{1}\right\|_{2}
$$

where $\mathrm{y}_{0}$ and $\mathrm{y}_{1}$ are the normalized $\mathrm{k}$-space data of the reference and the current processed temperature, respectively. As a dynamic procedure, the calculated scaling factor varies during heating. Therefore, the scaling factor computed based on Equation (8.9) is updated for every frame in the proposed model-based reconstruction.

\subsubsection{Regularization}

In the present work, as demonstrated in Equation (8.6), Tikhonov regularization is applied on the image content and the phase contrast map, respectively. According to the investigation in NLINV reconstruction $[1,41,114]$, in order to maintain the image quality of $\rho$, the damping factor $(p)$ is set to $0.7, \alpha_{n}$ is initialized to 1 and reduces by a 
factor of $1 / 2$ until the $7^{\text {th }}$ Newton step and then remains for the later Newton steps. As for the phase contrast map $(\Delta \phi)$, due to application of the high temporal resolution for data acquisition (200 ms per frame), only minor temperature changes appear between the neighboring frames. Therefore, $\Delta \phi$ is damped by 1 (i.e. $q=1$ ) to adopt more information from the previous acquisition and the corresponding regularization parameter $\beta_{n}$ is set to 1 which declines by a factor of $1 / 2$ for the first four Newton steps and remains until the reconstruction finishes. In addition, $\rho=1, \Delta \phi=0$ are used as the initial guesses for the reconstruction of the first frame and 8 Newton steps are employed in the present work.

\subsection{Methods and Materials}

\subsubsection{Numerical Simulation}

Numerical simulation was performed to assess the accuracy of the proposed model-based reconstruction for temperature mapping. The simulated images are shown in the left column of Figure 8.1. The reference and the post-heating datasets share the same magnitude, which consists of two superimposed ellipses. As for the phase information, the reference data had zero phase, while three small circles with different phase values were added in the post-heating data. According to Equation (3.9), temperature changes in the center part of the three circles were set to $20{ }^{\circ} \mathrm{C}, 12{ }^{\circ} \mathrm{C}$ and $5{ }^{\circ} \mathrm{C}$, respectively and that in the outer part of the circles had a gradient distribution to mimic the temperature during the laser heating procedure. Furthermore, the two datasets were transfered into k-space data via analytical Fourier transform with the following parameters: FOV $=128 \times 128$ $\mathrm{mm}^{2}$, in-plane resolution $=0.5 \times 0.5 \mathrm{~mm}^{2}$ and number of spokes $=17$. In order to simulate the long TE induced low SNR in the acquired data, high level of complex white Gaussian noise $(\mathrm{SD}=0.5)$ was added to every k-space spoke for both the reference and the post-heating data. Finally, according to the Biot-Savart law [115], ten coil sensitivity maps were also simulated to mimic parallel imaging.

\subsubsection{Laser Heating in Ex vivo Kidney}

In order to compare the proposed model-based method with the NLINV method, MR data obtained in Chapter 6.2.5 were employed for image reconstruction. Due to the memory limitation of the server system used for data processing, only 80 seconds of the original data were selected (10 seconds pre-heating and the first 70 seconds of laser heating). The corresponding laser parameters were: output power $=3 \mathrm{~W}$ and output energy $=56 \mathrm{~J}$. For post-processing, the median filter technique was applied on the magnitude and the temperature images generated by the NLINV method and on the magnitude images for the model-based reconstruction. 


\subsection{Results}

\subsubsection{Numerical Simulation}

Figure 8.1 illustrates the results of the numerical temperature phantom. Similar magnitude images are obtained from the model-based and the NLINV reconstructions. Different from the simulated magnitude image, the outer edge of the large ellipse has high signal intensity, due to the fact that ten receive coils are taken into account in the numerical simulation. In addition, the use of the temporal regularization on the phase difference $(\Delta \phi)$ in the model-based reconstruction leads to a temperature map with less noise compared with the NLINV method, especially in regions without temperature change or MR signal. Moreover, in the magnified temperature difference maps, the regions with temperature differences are better defined in the model-based reconstruction. Based on four chosen ROIs (indicated in Figure 8.1 by the dashed circles), the calculated temperature changes are compared with the true values in Table 8.1. Compared with the results of the NLINV reconstruction, the values of the model-based reconstruction are closer to the true temperature changes and have lower SDs.

Table 8.1.: Comparison between the temperature changes calculated by different methods.

\begin{tabular}{ccc}
\hline $\begin{array}{r}\text { True Temperature } \\
\text { Change }\left({ }^{\circ} \mathrm{C}\right)\end{array}$ & $\begin{array}{c}\text { Model-based } \\
\left({ }^{\circ} \mathrm{C}\right)\end{array}$ & $\begin{array}{c}\text { NLINV } \\
\left({ }^{\circ} \mathrm{C}\right)\end{array}$ \\
\hline 20 & $19.0 \pm 0.4$ & $18.5 \pm 0.7$ \\
12 & $11.2 \pm 0.4$ & $11.1 \pm 0.6$ \\
5 & $4.6 \pm 0.4$ & $4.6 \pm 0.8$ \\
0 & $0.0 \pm 0.3$ & $0.0 \pm 0.8$ \\
\hline
\end{tabular}

\subsubsection{Laser Heating Experiments}

Experimental results during laser heating in the swine kidney are shown in Figure 8.2. The proposed model-based and the NLINV reconstructions are performed on the same MR raw data. Because model-based reconstruction takes the reference and the current processed raw data into one signal model, the computed magnitude image has a better SNR than the image calculated from NLINV reconstruction. Additionally, similar to the results of numerical simulation, temperature values of $0{ }^{\circ} \mathrm{C}$ are observed in the temperature maps in regions without MR signal using the model-based reconstruction, whereas arbitrary values are observed in the same region with the NLINV method. More importantly, the magnified temperature map calculated by the model-based reconstruction, 


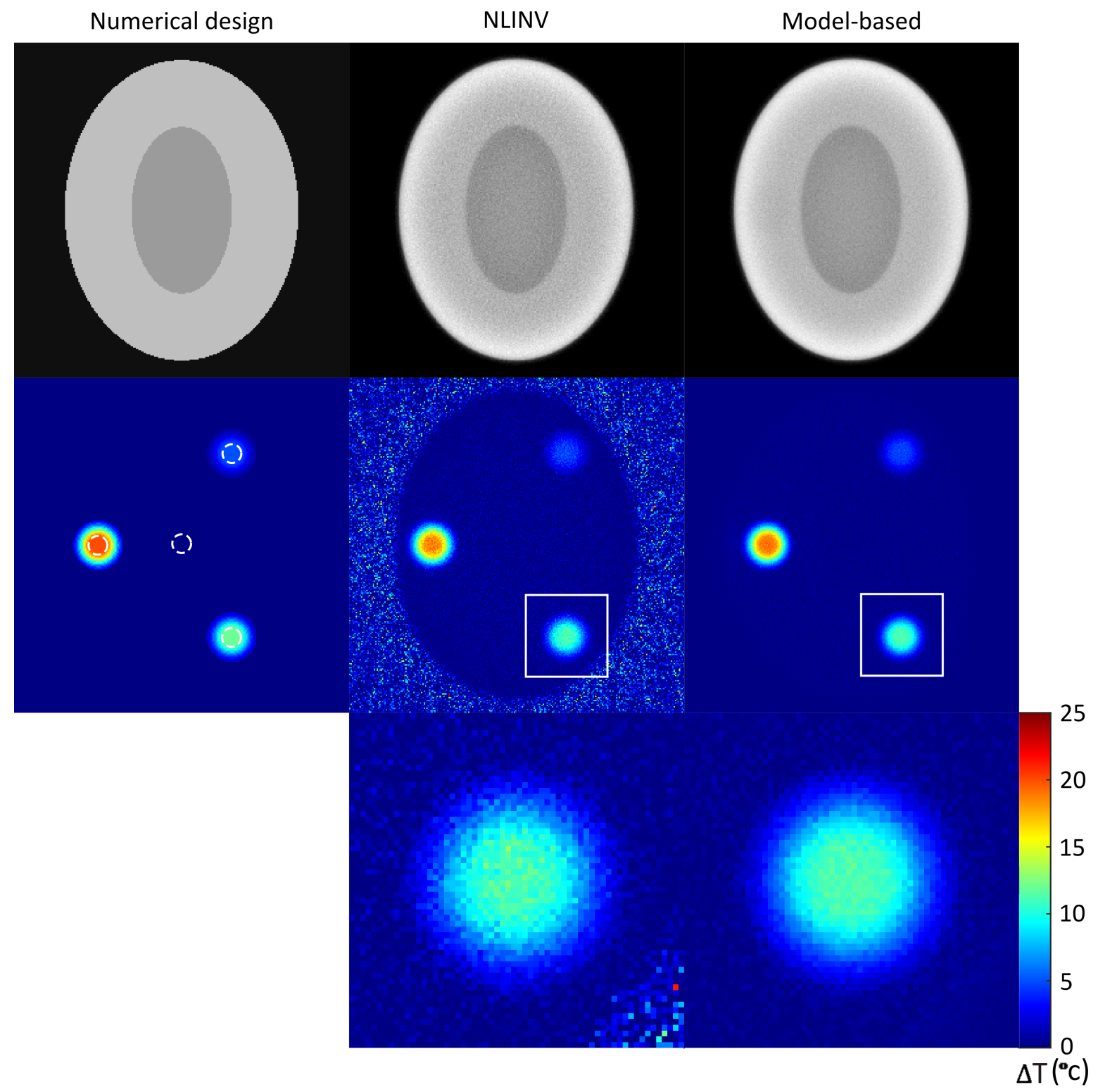

Figure 8.1.: Magnitude images and temperature difference maps of the numerical design (left column), NLINV reconstruction (middle column) and model-based reconstruction (right column). The corresponding k-space data is designed with 17 spokes per frame and complex white Gaussian noise $(\mathrm{SD}=0.5)$ is added to each spoke. The magnitude images reconstructed by model-based and NLINV methods are shown with the same color scale. Temperature values (mean $\pm \mathrm{SD}$ ) of the dashed circles are employed for the evaluation of the proposed reconstruction method (Table 8.1). 
which is acquired without applying median filter, shows the same image quality as that computed by the NLINV reconstruction. The temperature changes of the two ROIs of the heating process are presented in Figure 8.3. Model-based reconstruction yields slightly lower values than the NLINV method for the first 20 seconds of heating. Both methods show similar values for the rest of the heating period. In general, the model-based reconstruction results in a smoother curve of temperature change.

\subsection{Summary}

In this chapter, model-based reconstruction has been successfully performed for dynamic MR thermometry. The proposed signal model assumes that the temperature baseline and the current processed datasets share the same complex anatomical image and only differ in the temperature induced phase shift. The corresponding coil profiles are considered as known parameters, which are solved from the data acquired with the short TE, to reduce the total number of unknown variables in the nonlinear system. Further, in the cost function, two Tikhonov regularization terms with different parameters are used on the anatomical image and the phase difference map separately to ensure the smoothness of the phase contrast map. In conclusion, as shown in the numerical simulation, the proposed model-based reconstruction can provide accurate temperature values. In the $e x$ vivo experiments, this reconstruction offers anatomical images with higher SNR compared with the 'conventional' NLINV method. More importantly, it better defines the heated area and generates a smoother curve of the temperature changes during the heating procedure without the use of the median filter technique. Therefore, by applying the proposed model-based reconstruction, a true temperature updating time of $200 \mathrm{~ms}$ can be achieved for MR thermometry. 


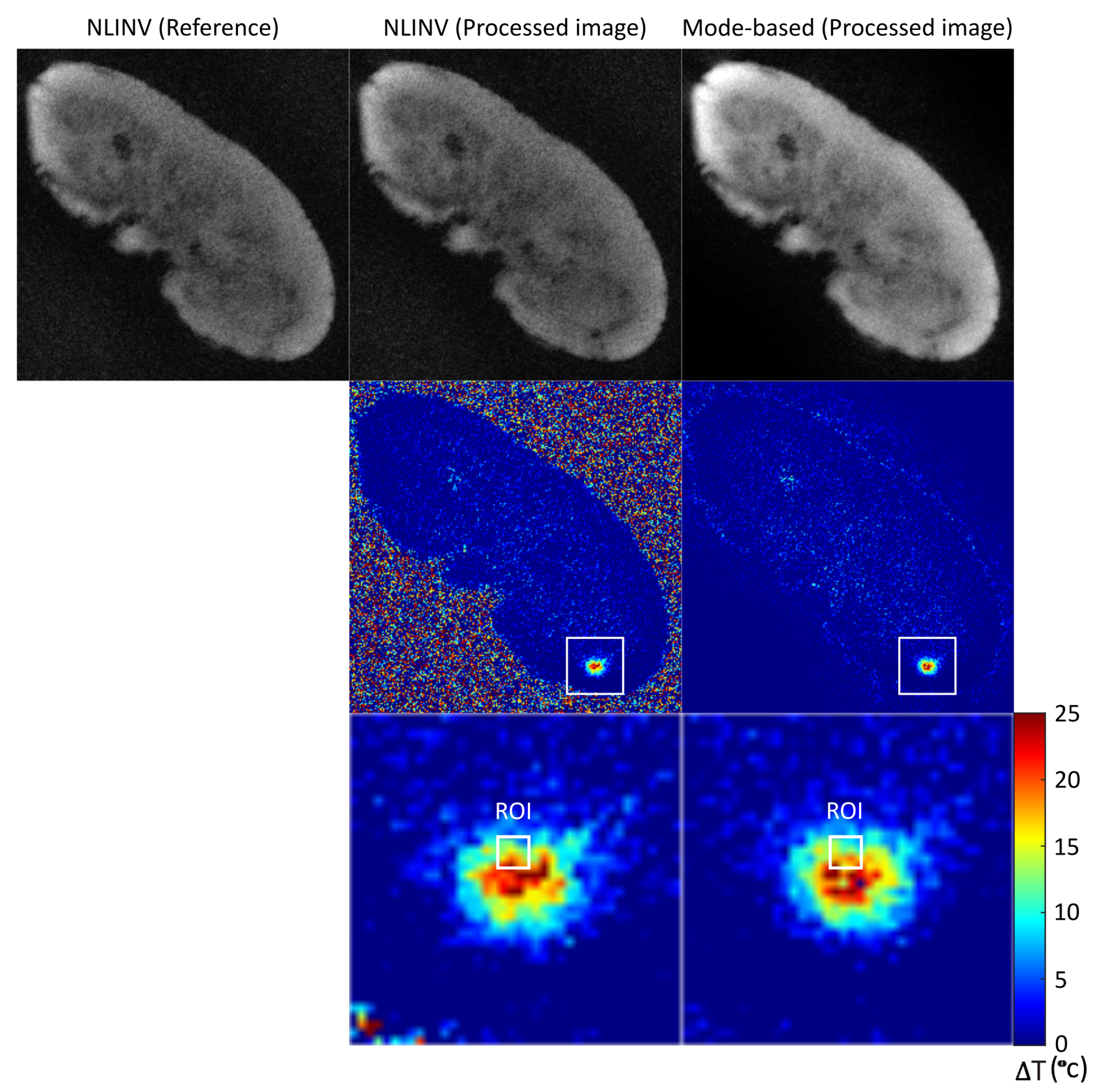

Figure 8.2.: Magnitude images and temperature difference maps of an excised swine kidney calculated by NLINV (left and middle columns) and model-based (right column) reconstruction during laser heating. NLINV method reconstructs the reference and the current processed data separately, while the model-based method calculates only one anatomical image from the two datasets. All the magnitude images are shown with the same color scale. The corresponding laser parameters are: output power $=3 \mathrm{~W}$ and output energy $=56 \mathrm{~J}$. The temperature change of the two ROIs next to the tip of the laser fiber is shown in Figure 8.3. 


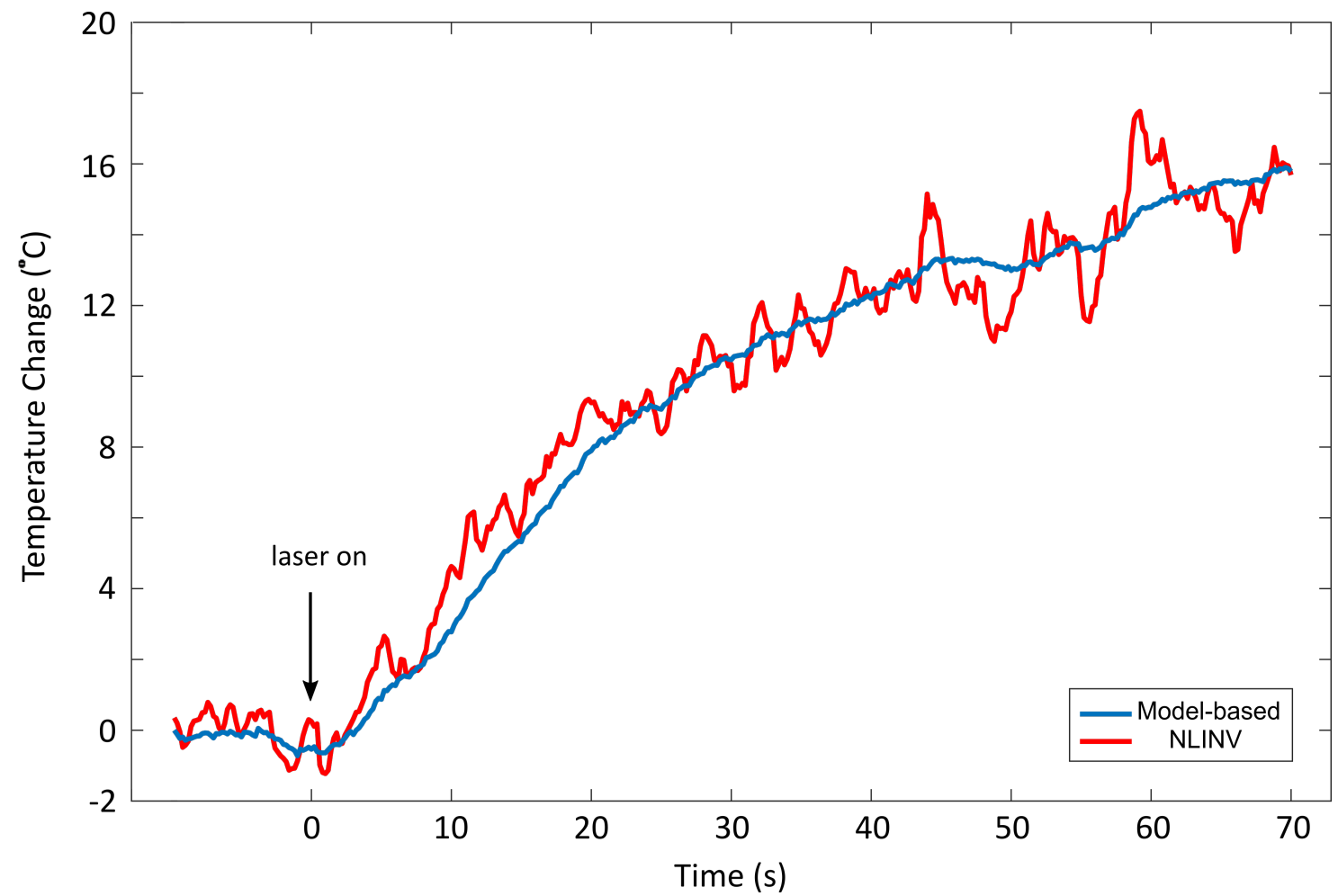

Figure 8.3.: Temperature changes of the chosen ROIs (depict in Figure 8.2) during laser heating calculated by the model-based (blue) and the NLINV (red) reconstruction. 



\section{Summary and Outlook}

\subsection{Summary}

In this thesis, dynamic MRI temperature mapping techniques were successfully implemented and validated for an experimental phantom as well as different ex vivo tissues. Rapid MRI-based thermometry is required to ensure the safety and efficacy of minimally invasive thermal therapies, which are of increasing clinical interest. The proposed methods were developed by exploiting recent advances in real-time MRI, which combine highly undersampled radial gradient-echo acquisition and nonlinear inverse (NLINV) image reconstruction. The results reveal the proton resonance frequency (PRF) as the preferred temperature-sensitive parameter and the most suitable technique for rapid MR thermometry. At this stage, a temporal resolution of $200 \mathrm{~ms}$ ( 5 frames per second) was achieved for a very high spatial resolution of $0.5 \times 0.5 \times 3 \mathrm{~mm}^{3}$.

As an initial step, a home-made MR-compatible phantom was constructed, which has an equivalent $T_{1}$ value as human tissues and can be heated from room temperature to about $40{ }^{\circ} \mathrm{C}$. Subsequently, PRF and $T_{1}$ thermometry were implemented based on modified real-time MRI. Optimizations were performed particularly with respect to radial data acquisition and NLINV image reconstruction. As a result of the phantom studies, both methods yielded dynamic temperature maps with satisfactory accuracy. However, experiments on excised swine kidneys confirmed the PRF method as a preferred temperature mapping technique for three reasons. Firstly, PRF thermometry, at least as developed in this work, resulted in a higher temporal resolution (200 ms) sufficient for capturing fast temperature changes as eventually achieved in a clinical scenario. Secondly, the temperature sensitivity of $\mathrm{PRF}$ is the same in all tissues with a dominant water proton signal (e.g., not fat). In contrast, the $T_{1}$ temperature sensitivity is tissue-dependent and has to be determined for every tissue of interest before the thermal therapy. Thirdly, the temperature uncertainty of PRF thermometry is much lower than that of $T_{1}$ thermometry. In a second step, a laser system was employed to generate focal heating in swine kidneys, which better mimicked thermal therapies. Real-time PRF MR thermometry successfully monitored corresponding temperature changes, while further sequence modifications allowed for the simultaneous acquisition of anatomic images with two different contrasts.

Because MR-based thermometry emerges as a relative measurement, all scans need a 


\section{Summary and Outlook}

reference. Under in vivo conditions this may lead to inter-scan errors due to motion. In this work, a reference selection procedure was developed, which establishes a library of reference scans by a multi-baseline acquisition and then performs an image similarity comparison to find a proper reference for the actual scan. The procedure was found to eliminate temperature errors in abdominal sections that are due to respiration-induced motions.

Based on the reference selection procedure, a model-based algorithm for phase-contrast MRI was adopted to temperature mapping. This novel method directly yields pairs of anatomic images and temperature difference maps from the undersampled sets of raw data without any intermediate image calculation. The proposed reconstruction combines the data of the reference and current scan into one signal model, which is defined by one anatomic image and and a corresponding phase difference map. Because only minor temperature changes occur between successive frames under high temporal resolution, the high strength of the Tikhonov regularization on the temperature difference map was maintained for all Newton steps. In comparison to NLINV reconstructions with subsequent complex image subtraction, the temperature maps present with improved spatial accuracy of heated areas, reduced noise in non-heated areas and a smoother temporal evolution of temperature changes.

\subsection{Outlook}

The observation of unexpected temperature errors in the kidney medulla in a region distant to focal heating suggests further experimental work. For example, the restriction of the data acquisition to a single imaging plane may be overcome by adding simultaneous multi-slice techniques [116]. These recent developments simultaneously excite and acquire MRI signals for several slices and therefore offer a better volume coverage both of the anatomic and temperature information without sacrificing the temporal resolution. Another limitation of the current study was the use of excised, i.e. non-perfused, swine kidneys that lack blood flow and tissue perfusion. Accordingly, the temperature development under in vivo conditions might behave differently. While passing the heated region, blood may partially cool the tissue and leave at higher temperature after absorbing thermal energy. Future studies of MR thermometry should therefore be performed on perfused organs or even better directly on anesthetized animals.

Another observation relates to 'black holes' near the tip of the laser fiber during heating. One interpretation of this phenomenon is due to the $2 \mu \mathrm{m}$ wavelength of the laser, which corresponds to a penetration depth of only $1 \mathrm{~mm}$ in aqueous tissues. As a consequence, extreme temperature changes are achieved within the penetration depth that cause tissue 
ablation and even generate steam bubbles. Therefore, the use of a laser system with an extended penetration depth (e.g., $10 \mathrm{~mm}$ ) may turn out to be beneficial, because it can not only avoid the high power density, but also maintain the desired temperature changes $[105,117,118]$.

Thirdly, the phantom and ex vivo experiments in this thesis were performed with use of a 64-channel head coil in a $3 \mathrm{~T}$ MRI system. However, in order to monitor minimally invasive thermal therapies in the human heart and abdomen, other RF coils with a lower number of less sensitive receive channels have to be employed to cover the desired FOV [113]. As a consequence, the spatial and/or temporal resolution might be decreased to not sacrifice image quality. However, based on previous experience with real-time MRI techniques as used here [39, 41], sufficient spatiotemporal resolution may still be expected for MRI thermometry. A similar promise holds true for the in vivo motion problem which may be overcome by the reference selection procedure proposed in this work.

Finally, as for the model-based reconstruction, the strong temporal regularization used here for the temperature difference map may cause the accumulation of noise in nonheated regions with increasing measurement time. One possible solution might be the evaluation of other regularization techniques, such as a wavelet-based $l_{1}$ regularization [119], and its integration into the iterative Gauss-Newton algorithm. This is because the $l_{1}$ regularization more efficiently suppresses noise when applied on sparse images after suitable transformation.

Taken together, dynamic MRI temperature mapping as developed in this thesis offers accurate relative temperature maps as well as anatomic images with high spatiotemporal resolution. The current results provide a systematic background for future clinical trials. In general, real-time PRF thermometry offers a huge potential to contribute to minimally invasive thermal therapies guided and monitored by MRI. 



\section{A. Calculation of Bio-heat Transfer Model}

According to Equation (3.2), the heating procedure of a thermal therapy can be expressed as:

$$
\frac{\partial \hat{T}(\vec{r}, t)}{\partial t}=D \nabla^{2} \hat{T}(\vec{r}, t)-w_{b} \hat{T}(\vec{r}, t)+\alpha p(\vec{r})
$$

The integral of Equation (A.1) over $\vec{r}$ yields the following function:

$$
\frac{\partial E_{t h}(t)}{\partial t}=D \int_{\vec{r}} \nabla^{2} \hat{T}(\vec{r}, t) d \vec{r}-w_{b} E_{t h}(t)+\alpha \int_{\vec{r}} p(\vec{r}) d \vec{r}
$$

with $E_{t h}$ being the total thermal load over the treated volume. Note that the integration of $p(\vec{r})$ in Equation (A.2) is the total power $\left(p_{t}\right)$ absorbed by heated tissue and the corresponding mathematical expression is :

$$
p_{t}=\int_{\vec{r}} p(\vec{r}) d \vec{r}
$$

Consider the heated region is located in a sufficiently large spherical coordinate with a radius of $\vec{R}$, the boundary conditions of Equation (A.2) is:

$$
\begin{aligned}
\hat{T}(\vec{R}, t) & =0 \\
\frac{\partial \hat{T}(\vec{R}, t)}{\partial r} & =0
\end{aligned}
$$

which leads $\int_{\vec{r}} \nabla^{2} \hat{T}(\vec{r}, t) d \vec{r}$ to 0 . Therefore, Equation (A.2) can be simplified as the following differential equation:

$$
w_{b} E_{t h}(t)+\frac{\partial E_{t h}(t)}{\partial t}=\alpha p_{t}
$$

with the analytical solution expressed as: 
Appendix A. Calculation of Bio-heat Transfer Model

$$
E_{t h}= \begin{cases}\frac{\alpha p_{t}\left[1-e^{\left(-w_{b} t\right)}\right]}{w_{b}} & t \leq t_{h} \\ E_{t h 0} e^{\left(-w_{b}\left(t-t_{h}\right)\right)} & t>t_{h}\end{cases}
$$

with $t_{h}$ being the total heating period, $E_{t h 0}$ representing maximum thermal load when $t=t_{h}$. 


\section{Bibliography}

[1] M Uecker, S Zhang, and J Frahm. Nonlinear inverse reconstruction for real-time MRIof the human heart using undersampled radial FLASH. Magn Reson Med, 63:1456-1462, 2010.

[2] MA Bernstein, KF King, and XJ Zhou. Handbook of MRI pulse sequences. Elsevier, 2004.

[3] EM Haacke, RW Brown, MR Thompson, and R Venkatesan. Magnetic resonance imaging: physical principles and sequence design. John Wiley \& Sons, 1999.

[4] F Bloch. Nuclear induction. Phys Rev, 70:460, 1946.

[5] EM Purcell, HC Torrey, and RV Pound. Resonance absorption by nuclear magnetic moments in a solid. Phys Rev, 69:37, 1946.

[6] Z Liang and PC Lauterbur. Principles of magnetic resonance imaging: a signal processing approach, 2000.

[7] DB Plewes and W Kucharczyk. Physics of MRI: a primer. J Magn Reson Imaging, 35:1038-1054, 2012.

[8] J Frahm, A Haase, and D Matthaei. Rapid NMR imaging of dynamic processes using the FLASH technique. Magn Reson Med, 3:321-327, 1986.

[9] A Haase, J Frahm, D Matthaei, W Hänicke, and KD Merboldt. FLASH imaging. rapid NMR imaging using low flip-angle pulses. J Magn Reson, 67:258-266, 1986.

[10] PC Lauterbur. Image formation by induced local interactions: examples employing nuclear magnetic resonance. Nature, 242:190-191.

[11] P Mansfield. Multi-planar image formation using NMR spin echoes. Journal of Physics C: Solid State Physics, 10:L55, 1977.

[12] P Mansfield and IL Pykett. Biological and medical imaging by NMR. 1. J Magn Reson, 355-377, 1978.

[13] CB Ahn, JH Kim, and ZH Cho. High-speed spiral-scan echo planar NMR imaging-i. IEEE Trans Med Imaging, 5:2-7, 1986. 
[14] DC Noll, JD Cohen, CH Meyer, and W Schneider. Spiral k-space MR imaging of cortical activation. J Magn Reson Imaging, 5:49-56, 1995.

[15] JW Cooley and JW Tukey. An algorithm for the machine calculation of complex fourier series. Mathematics of computation, 19:297-301, 1965.

[16] RE Hendrick, JB Kneeland, and DD Stark. Maximizing signal-to-noise and contrast-to-noise ratios in FLASH imaging. Magn Reson Imaging, 5:117-127, 1987.

[17] HY Carr. Steady-state free precession in nuclear magnetic resonance. Phys Rev, 112:1693, 1958.

[18] AP Crawley, ML Wood, and RM Henkelman. Elimination of transverse coherences in FLASH MRI. Magn Reson Med, 8:248-260, 1988.

[19] J Frahm, W Hänicke, and KD Merboldt. Transverse coherence in rapid FLASH NMR imaging. J Magn Reson, 72:307-314, 1987.

[20] KT Block. Advanced methods for radial data sampling in magnetic resonance imaging. SUB University of Goettingen, 2008.

[21] S Zhang, KT Block, and J Frahm. Magnetic resonance imaging in real time: advances using radial FLASH. J Magn Reson Imaging, 31:101-109, 2010.

[22] KT Block and M Uecker. Simple method for adaptive gradient-delay compensation in radial MRI. In Proceedings of the 19th Annual Meeting of ISMRM, Montreal, Canada, page 2816, 2011.

[23] SJ Riederer, T Tasciyan, F Farzaneh, JN Lee, RC Wright, and RJ Herfkens. MR fluoroscopy: technical feasibility. Magn Reson Med, 8:1-15, 1988.

[24] JP Debbins, SJ Riederer, PJ Rossman, RC Grimm, JP Felmlee, JF Breen, and RL Ehman. Cardiac magnetic resonance fluoroscopy. Magn Reson Med, 36:588595, 1996.

[25] JD O'Sullivan. A fast sinc function gridding algorithm for fourier inversion in computer tomography. IEEE Trans Med Imaging, 4:200-207, 1985.

[26] JI Jackson, CH Meyer, DG Nishimura, and A Macovski. Selection of a convolution function for fourier inversion using gridding [computerised tomography application]. IEEE Trans Med Imaging, 10:473-478, 1991.

[27] V Rasche, R Proksa, R Sinkus, P Börnert, and H Eggers. Resampling of data between arbitrary grids using convolution interpolation. IEEE Trans Med Imaging, 18:385-392, 1999. 
[28] PJ Beatty, DG Nishimura, and JM Pauly. Rapid gridding reconstruction with a minimal oversampling ratio. IEEE Trans Med Imaging, 24:799-808, 2005.

[29] JA Fessler and BP Sutton. Nonuniform fast fourier transforms using min-max interpolation. IEEE Trans Signal Processing, 51:560-574, 2003.

[30] PB Roemer, WA Edelstein, CE Hayes, SP Souza, and OM Mueller. The NMR phased array. Magn Reson Med, 16:192-225, 1990.

[31] DK Sodickson and WJ Manning. Simultaneous acquisition of spatial harmonics (SMASH): fast imaging with radiofrequency coil arrays. Magn Reson Med, 38:591603, 1997.

[32] MA Griswold, PM Jakob, RM Heidemann, M Nittka, V Jellus, J Wang, B Kiefer, and A Haase. Generalized autocalibrating partially parallel acquisitions (GRAPPA). Magn Reson Med, 47:1202-1210, 2002.

[33] KP Pruessmann, M Weiger, MB Scheidegger, and P Boesiger. Sense: sensitivity encoding for fast MRI. Magn Reson Med, 42:952-962, 1999.

[34] KP Pruessmann, M Weiger, P Börnert, and P Boesiger. Advances in sensitivity encoding with arbitrary k-space trajectories. Magn Reson Med, 46:638-651, 2001.

[35] F Bauer and S Kannengiesser. An alternative approach to the image reconstruction for parallel data acquisition in MRI. Math Meth Appl Sci, 30:1437-1451, 2007.

[36] M Uecker, T Hohage, KT Block, and J Frahm. Image reconstruction by regularized nonlinear inversion - joint estimation of coil sensitivities and image content. Magn Reson Med, 60:674-682, 2008.

[37] M Uecker, S Zhang, D Voit, A Karaus, KD Merboldt, and J Frahm. Real-time MRI at a resolution of 20 ms. NMR Biomed, 23:986-994, 2010.

[38] S Schaetz and M Uecker. A multi-GPU programming library for real-time applications. In Algorithms and Architectures for Parallel Processing, pages 114-128. Springer, 2012.

[39] M Uecker, S Zhang, D Voit, KD Merboldt, and J Frahm. Real-time MRI: recent advances using radial FLASH. Imaging Med, 4:461-476, 2012.

[40] A Niebergall, S Zhang, E Kunay, G Keydana, M Job, M Uecker, and J Frahm. Real-time MRI of speaking at a resolution of 33 ms: Undersampled radial FLASH with nonlinear inverse reconstruction. Magn Reson Med, 69:477-485, 2013.

[41] AA Joseph, KD Merboldt, D Voit, S Zhang, M Uecker, J Lotz, and J Frahm. Realtime phase-contrast MRI of cardiovascular blood flow using undersampled radial fast low-angle shot and nonlinear inverse reconstruction. 
[42] X Wang, VB Roeloffs, KD Merboldt, D Voit, S Schätz, and J Frahm. Single-shot multi-slice T1 mapping at high spatial resolution-inversion-recovery FLASH with radial undersampling and iterative reconstruction. Open Med Imaging J, 9:1-8, 2015.

[43] CT Germer, CM Isbert, D Albrecht, JP Ritz, A Schilling, A Roggan, KJ Wolf, G Müller, and HJ Buhr. Laser-induced thermotherapy for the treatment of liver metastasis. Surg Endoscopy, 12:1317-1325, 1998.

[44] KK Vigen, J Jarrard, V Rieke, J Frisoli, BL Daniel, and KBs Pauly. In vivo porcine liver radiofrequency ablation with simultaneous MR temperature imaging. J Magn Reson Imaging, 23:578-584, 2006.

[45] SL Hokland, M Pedersen, R Salomir, B Quesson, H Stodkilde, and CT Moonen. MRI-guided focused ultrasound: methodology and applications. IEEE Trans Med Imaging, 25:723-731, 2006.

[46] O Svelto and DC Hanna. Principles of lasers. 1998.

[47] A L Schawlow and CH Townes. Infrared and optical masers. Phys Rev, 112:1940, 1958.

[48] JH Kim and EW Hahn. Clinical and biological studies of localized hyperthermia. Cancer Res, 39:2258-2261, 1979.

[49] S Thomsen. Pathologic analysis of photothermal and photomechanical effects of laser-tissue interactions. Photochem and Photobiol, 53:825-835, 1991.

[50] S Becker and A Kuznetsov. Heat Transfer and Fluid Flow in Biological Processes. Academic Press, 2014.

[51] J Heisterkamp, R van Hillegersberg, and J Jzermans. Critical temperature and heating time for coagulation damage: implications for interstitial laser coagulation (ILC) of tumors. Laser Surg Med, 25:257-262, 1999.

[52] HH Pennes. Analysis of tissue and arterial blood temperatures in the resting human forearm. J Appl Physiol, 1:93-122, 1948.

[53] I Dragonu, PL de Oliveira, C Laurent, C Mougenot, N Grenier, CT Moonen, and B Quesson. Non-invasive determination of tissue thermal parameters from high intensity focused ultrasound treatment monitored by volumetric MRI thermometry. NMR Biomed, 22:843-851, 2009.

[54] F Cornelis, N Grenier, CT Moonen, and B Quesson. In vivo characterization of tissue thermal properties of the kidney during local hyperthermia induced by MRguided high-intensity focused ultrasound. NMR Biomed, 24:799-806, 2011. 
[55] JC Hindman. Proton resonance shift of water in the gas and liquid states. J Chem Phys, 44:4582-4592, 1966.

[56] WG Schneider, HJ Bernstein, and JA Pople. Proton magnetic resonance chemical shift of free (gaseous) and associated (liquid) hydride molecules. J Chem Phys, 28:601-607, 1958.

[57] N Muller and RC Reiter. Temperature dependence of chemical shifts of protons in hydrogen bonds. J Chem Phys, 42:3265-3269, 1965.

[58] Y Ishihara, A Calderon, H Watanabe, K Okamoto, Y Suzuki, K Kuroda, and Y Suzuki. A precise and fast temperature mapping using water proton chemical shift. Magn Reson Med, 34:814-823, 1995.

[59] JD Poorter, CD Wagter, YD Deene, C Thomsen, F Ståhlberg, and E Achten. Noninvasive MRI thermometry with the proton resonance frequency (PRF) method: in vivo results in human muscle. Magn Reson Med, 33:74-81, 1995.

[60] JD Poorter. Noninvasive MRI thermometry with the proton resonance frequency method: study of susceptibility effects. Magn Reson Med, 34:359-367, 1995.

[61] RT Peters, RS Hinks, and RM Henkelman. Ex vivo tissue-type independence in proton-resonance frequency shift mr thermometry. Magn Reson Med, 40:454-459, 1998.

[62] R Stollberger, PW Ascher, D Huber, W Renhart, H Radner, and F Ebner. Temperature monitoring of interstitial thermal tissue coagulation using MR phase images. J Magn Reson Imaging, 8:188-196, 1998.

[63] K Kuroda, K Oshio, RV Mulkern, and FA Jolesz. Optimization of chemical shift selective suppression of fat. Magn Reson Med, 40:505-510, 1998.

[64] K Hynynen, N McDannold, RV Mulkern, and FA Jolesz. Temperature monitoring in fat with mri. Magn Reson Med, 43:901-904, 2000.

[65] N Bloembergen, EM Purcell, and RV Pound. Relaxation effects in nuclear magnetic resonance absorption. Phys Rev, 73:679, 1948.

[66] DL Parker, V Smith, P Sheldon, LE Crooks, and L Fussell. Temperature distribution measurements in two-dimensional NMR imaging. Med Phys, 10:321-325, 1983.

[67] PA Bottomley, TH Foster, RE Argersinger, and L Pfeifer. A review of normal tissue hydrogen NMR relaxation times and relaxation mechanisms from 1-100 mhz: dependence on tissue type, NMR frequency, temperature, species, excision, and age. Med Phys, 11:425-448, 1984. 
[68] TR Nelson and SM Tung. Temperature dependence of proton relaxation times in vitro. Magn Reson Imaging, 5:189-199, 1987.

[69] CJ Lewa and Z Majewska. Temperature relationships of proton spin-lattice relaxation time T1 in biological tissues. Bulletin du cancer, 67:525-530, 1979.

[70] HE Cline, K Hynynen, CJ Hardy, RD Watkins, JF Schenck, and FA Jolesz. MR temperature mapping of focused ultrasound surgery. Magn Reson Med, 31:628-636, 1994.

[71] R Matsumoto, K Oshio, and FA Jolesz. Monitoring of laser and freezinginduced ablation in the liver with T1-weighted MR imaging. J Magn Reson Imaging, 2:555$562,1992$.

[72] SJ Graham, MJ Bronskill, and RM Henkelman. Time and temperature dependence of MR parameters during thermal coagulation of ex vivo rabbit muscle. Magn Reson Med, 39:198-203, 1998.

[73] IR Young, JW Hand, A Oatridge, and MV Prior. Modeling and observation of temperature changes in vivo using mri. Magn Reson Med, 32:358-369, 1994.

[74] TJ Vogl, R Straub, S Zangos, MG Mack, and K Eichler. Mr-guided laser-induced thermotherapy (LITT) of liver tumours: experimental and clinical data. Int J Hypertherm, 20:713-724, 2004.

[75] V Rieke and Butts PK. Mr thermometry. J Magn Reson Imaging, 27:376-390, 2008.

[76] B Quesson, J A de Zwart, and CT Moonen. Magnetic resonance temperature imaging for guidance of thermotherapy. J Magn Reson Imaging, 12:525-533, 2000.

[77] B de Senneville, B Quesson, and CT Moonen. Magnetic resonance temperature imaging. International journal of hyperthermia, 21:515-531, 2005.

[78] FH Johnson, H Eyring, and BJ Stover. The theory of rate processes in biology and medicine. Wiley-Interscience, 1974.

[79] D Le Bihan, J Delannoy, and RL Levin. Temperature mapping with MR imaging of molecular diffusion: application to hyperthermia. Radiology, 171:853-857, 1989.

[80] Y Zhang, TV Samulski, WT Joines, J Mattiello, RL Levin, and D LeBihan. On the accuracy of noninvasive thermometry using molecular diffusion magnetic resonance imaging. International journal of hyperthermia, 8:263-274, 1992.

[81] MD Mitchell, HL Kundel, L Axel, and PM Joseph. Agarose as a tissue equivalent phantom material for NMR imaging. Magn Reson Imaging, 4:263-266, 1986. 
[82] KA Kraft, PP Fatouros, GD Clarke, and PRS Kishore. An MRI phantom material for quantitative relaxometry. Magn Reson Med, 5:555-562, 1987.

[83] FA Howe. Relaxation times in paramagnetically doped agarose gels as a function of temperature and ion concentration. Magn Reson Imaging, 6:263-270, 1988.

[84] P Walker, RA Lerski, R Mathur, J Binet, and F Yane. Vi. preparation of agarose gels as reference substances for NMR relaxation time measurement. Magn Reson Imaging, 6:215-222, 1988.

[85] Y Zur, ML Wood, and LJ Neuringer. Spoiling of transverse magnetization in steady-state sequences. Magn Reson Med, 21:251-263, 1991.

[86] AH Chung, K Hynynen, V Colucci, K Oshio, HE Cline, and FA Jolesz. Optimization of spoiled gradient-echo phase imaging for in vivo localization of a focused ultrasound beam. Magn Reson Med, 36:745-752, 1996.

[87] EM Haacke and GW Lenz. Improving MR image quality in the presence of motion by using rephasing gradients. Am J Roentgenol, 148:1251-1258, 1987.

[88] PM Pattany, JJ Phillips, LC Chiu, JD Lipcamon, JL Duerk, JM McNally, and SN Mohapatra. Motion artifact suppression technique (MAST) for MR imaging. JCAT, 11:369-377, 1987.

[89] JA de Zwart, P van Gelderen, DJ Kelly, and CT Moonen. Fast magnetic-resonance temperature imaging. J Magn Reson, Series B, 112:86-90, 1996.

[90] E Quaia. Radiological Imaging of the kidney. Springer, 2014.

[91] MA Bernstein, M Grgic, TJ Brosnan, and NJ Pelc. Reconstructions of phase contrast, phased array multicoil data. Magn Reson Med, 32:330-334, 1994.

[92] DC Look and DR Locker. Time saving in measurement of NMR and EPR relaxation times. Review of Scientific Instruments, 41:250-251, 1970.

[93] R Deichmann and A Haase. Quantification of T1 values by snapshot-flash NMR imaging. J Magn Reson, 96:608-612, 1992.

[94] A Tannús and M Garwood. Adiabatic pulses. NMR Biomed, 10:423-434, 1997.

[95] V Roeloffs, D Voit, and J Frahm. Spoiling without additional gradients: Radial FLASH MRI with randomized radiofrequency phases. Magn Reson Med, 2015.

[96] S Hofer, X Wang, V Roeloffs, and J Frahm. Single-shot T1 mapping of the corpus callosum: a rapid characterization of fiber bundle anatomy. Front Neuroanatomy, $9,2015$. 
[97] J Klosowski and J Frahm. Image denoising for real-time MRI. Magn Reson Med, 2016.

[98] CJ Weight, JH Kaouk, NJ Hegarty, EM Remer, CM O’Malley, BR Lane, IS Gill, and AC Novick. Correlation of radiographic imaging and histopathology following cryoablation and radio frequency ablation for renal tumors. J Urology, 179:1277$1283,2008$.

[99] C Damianou, M Pavlou, O Velev, K Kyriakou, and M Trimikliniotis. High intensity focused ultrasound ablation of kidney guided by MRI. Ultrasound Med Biol, 30:397404, 2004.

[100] J Hindley, WM Gedroyc, L Regan, E Stewart, C Tempany, K Hynnen, N Macdanold, Y Inbar, Y Itzchak, and J Rabinovici. MRI guidance of focused ultrasound therapy of uterine fibroids: early results. Am J Roentgenol, 183:1713-1719, 2004.

[101] SG Silverman, K Tuncali, DF Adams, E van Sonnenberg, KH Zou, DF Kacher, PR Morrison, and FA Jolesz. MR imaging-guided percutaneous cryotherapy of liver tumors: Initial experience 1. Radiology, 217:657-664, 2000.

[102] DP O'Regan, MF Callaghan, M Wylezinska-Arridge, J Fitzpatrick, RP Naoumova, JV Hajnal, and SA Schmitz. Liver fat content and T2*: Simultaneous measurement by using breath-hold multiecho MR imaging at $3.0 \mathrm{~T}$-feasibility 1. Radiology, 247:550-557, 2008.

[103] T Kahn and H Busse. Interventional Magnetic Resonance Imaging. Springer, 2012.

[104] K Scholle, P Fuhrberg, P Koopmann, and S Lamrini. $2 \mu \mathrm{m}$ laser sources and their possible applications. INTECH Open Access Publisher, 2010.

[105] A Carpentier, RJ McNichols, RJ Stafford, JP Guichard, D Reizine, S Delaloge, E Vicaut, D Payen, A Gowda, and B George. Laser thermal therapy: Real-time MRI-guided and computer-controlled procedures for metastatic brain tumors. Laser Surg Med, 43:943-950, 2012.

[106] S Morikawa, T Inubushi, Y Kurumi, S Naka, K Sato, K Demura, T Tani, and HA Haque. Feasibility of respiratory triggering for MR-guided microwave ablation of liver tumors under general anesthesia. Cardiovasc Intervent Radiology, 27:370373, 2004.

[107] JA de Zwart, FC Vimeux, J Palussiere, R Salomir, B Quesson, C Delalande, and CT Moonen. On-line correction and visualization of motion during MRI-controlled hyperthermia. Magn Reson Med, 45:128-137, 2001. 
[108] KK Vigen, BL Daniel, JM Pauly, and K Butts. Triggered, navigated, multi-baseline method for proton resonance frequency temperature mapping with respiratory motion. Magn Reson Med, 50:1003-1010, 2003.

[109] V Rieke, KK Vigen, G Sommer, BL Daniel, JM Pauly, and K Butts. Referenceless prf shift thermometry. Magn Reson Med, 51:1223-1231, 2004.

[110] K Kuroda, D Kokuryo, E Kumamoto, K Suzuki, Y Matsuoka, and B Keserci. Optimization of self-reference thermometry using complex field estimation. Magn Reson Med, 56:835-843, 2006.

[111] C Weidensteiner, N Kerioui, B Quesson, BD de Senneville, H Trillaud, and CT Moonen. Stability of real-time MR temperature mapping in healthy and diseased human liver. J Magn Reson Imaging, 19:438-446, 2004.

[112] BD De Senneville, C Mougenot, and CT Moonen. Real-time adaptive methods for treatment of mobile organs by mri-controlled high-intensity focused ultrasound. Magn Reson Med, 57:319-330, 2007.

[113] S Roujol, M Ries, B Quesson, CT Moonen, and B de Senneville. Real-time MRthermometry and dosimetry for interventional guidance on abdominal organs. Magn Reson Med, 63:1080-1087, 2010.

[114] Z Tan, V Roeloffs, D Voit, AA Joseph, M Untenberger, KD Merboldt, and J Frahm. Model-based reconstruction for real-time phase-contrast flow MRI: Improved spatiotemporal accuracy. Magn Reson Med, 2016.

[115] M Guerquin-Kern, L Lejeune, KP Pruessmann, and M Unser. Realistic analytical phantoms for parallel magnetic resonance imaging. IEEE Trans Med Imaging, 31:626-636, 2012.

[116] M Barth, F Breuer, PJ Koopmans, DG Norris, and BA Poser. Simultaneous multislice (sms) imaging techniques. Magn Reson Med, 75:63-81, 2016.

[117] TJ Vogl, R Straub, K Eichler, O Söllner, and MG Mack. Colorectal carcinoma metastases in liver: Laser-induced interstitial thermotherapy-local tumor control rate and survival data. Radiology, 230:450-458, 2004.

[118] MG de Jode, JA Vale, and WM Gedroyc. MR-guided laser thermoablation of inoperable renal tumors in an open-configuration interventional MR scanner: Preliminary clinical experience in three cases. J Magn Reson Imaging, 10:545-549, 1999.

[119] M Guerquin-Kern, M Haberlin, KP Pruessmann, and M Unser. A fast waveletbased reconstruction method for magnetic resonance imaging. IEEE Trans Med Imaging, 30:1649-1660, 2011. 



\section{Curriculum Vitae}

\section{Personal Data}

$\begin{array}{ll}\text { Name: } & \text { Zhongshuai Zhang } \\ \text { Date of Birth: } & \text { July 20, 1985 } \\ \text { Place of Birth: } & \text { Changchun, China } \\ \text { Nationality: } & \text { Chinese } \\ \text { Email: } & \text { zzhang@gwdg.de }\end{array}$

\section{Education}
2004-2008 Bachelor Degree, Physics, Northeast Normal University, Changchun, China
2009 - 2012 Master Degree, Nuclear Technology, Northeast Normal University, Changchun, China
2012 - present PhD student, Max Planck Institute for Biophysical Chemistry, Göttingen, Germany

\section{Research Experiences}

2008 Bachelor thesis: The Transmission of Sound in a Vacuum System 2009 - $2012 \quad$ Master thesis: Properties of the Associated Particle Sealed-tube Neutron Generator

2012 - present PhD thesis: Dynamic Temperature Mapping - Real-time MRI Strategies and Model-based Reconstructions

\section{Teaching Experiences}

$\begin{array}{ll}2008-2009 & \text { Volunteer teacher, No.2 Middle School of Zhongning County } \\ 2013-2015 & \text { Lecturer, Basic Principle of MRI and Real-time MRI, Göttingen } \\ & \text { Graduate School for Neurosciences, Biophysics and Molecular Bio- } \\ & \text { sciences, Max Planck Institute for Biophysical Chemistry } \\ & \text { Teaching assistant, Advanced Mathematics, Faculty of Physics, } \\ & \text { University Göttingen }\end{array}$

